\title{
Group Actions on Trees
}

by

Ming Ming Zhang, B. Math. (Honours)

A thesis Submitted to the Faculty of Graduate Studies and Research in partial fulfilment of the requirements for the degree of Master

Ottawa-Carleton Institute Department of Mathematics and Statistics Carleton University

Ottawa, Ontario, Canada

August, 2009

(C) Copyright 2009 
Library and Archives

Canada

Published Heritage

Branch

395 Wellington Street

Ottawa ON K1A ON4

Canada
Bibliotheque et

Archives Canada

Direction du

Patrimoine de l'édition

395, rue Wellington

Ottawa ON K1A ON4

Canada
Your file Votre refférence

ISBN: 978-0-494-60210-2

Our file Notre reférence

ISBN: 978-0-494-60210-2
NOTICE:

The author has granted a nonexclusive license allowing Library and Archives Canada to reproduce, publish, archive, preserve, conserve, communicate to the public by telecommunication or on the Internet, loan, distribute and sell theses worldwide, for commercial or noncommercial purposes, in microform, paper, electronic and/or any other formats.

The author retains copyright ownership and moral rights in this thesis. Neither the thesis nor substantial extracts from it may be printed or otherwise reproduced without the author's permission.
AVIS:

L'auteur a accordé une licence non exclusive permettant à la Bibliothèque et Archives Canada de reproduire, publier, archiver, sauvegarder, conserver, transmettre au public par télécommunication ou par l'Internet, prêter, distribuer et vendre des thèses partout dans le monde, à des fins commerciales ou autres, sur support microforme, papier, électronique et/ou autres formats.

L'auteur conserve la propriété du droit d'auteur et des droits moraux qui protège cette thèse. Ni la thèse ni des extraits substantiels de celle-ci ne doivent être imprimés ou autrement reproduits sans son autorisation.
In compliance with the Canadian Privacy Act some supporting forms may have been removed from this thesis.

While these forms may be included in the document page count, their removal does not represent any loss of content from the thesis.
Conformément à la loi canadienne sur la protection de la vie privée, quelques formulaires secondaires ont été enlevés de cette thèse.

Bien que ces formulaires aient inclus dans la pagination, il n'y aura aucun contenu manquant. 


\section{Abstract}

We give a topological approach for the study of the algebraic structure of subgroups of free products, amalgamated free products and HNN extensions. We also prove that a stable action finitely presented group $G$ on an $\mathbb{R}$-tree gives a resolving band complex which decomposes $G$. 


\title{
Group Actions on Trees
}

\author{
Ming Ming Zhang
}

Master's thesis August 17, 2009

Department of Mathematics and Statistics Carleton University 


\title{
Groups action on trees
}

Ming Ming Zhang

Department of Mathematics and Statistics

Herzberg Building, Carleton University

1125 Colonel By Drive

Ottawa, ON CANADA K1S 5B6

Master's thesis

\begin{abstract}
We give a topological approach for the study of the algebraic structure of subgroups of free products, amalgamated free products and HNN extensions. We also prove that a stable action finitely presented group $G$ on an $\mathbb{R}$-tree gives a resolving band complex which decomposes $G$.
\end{abstract}

\section{Acknowledgement}

I am deeply grateful to my thesis advisor Professor Inna Bumagina for her valuable advice, help and encouragement. I also wish to thank Dr. Mahmood Sohrabi for his valuable remarks enabled me to understand the Rips theory well.

\section{Contents}

1 Introduction 1

2 Amalgamated Free Products and HNN Extensions 2

2.1 Some basic definitions . . . . . . . . . . . . . . 2

2.2 Definitions of amalgamated free products and HNN extensions . . . 8

2.3 Grushko's theorem . . . . . . . . . . . . . . . 9

2.4 Applications of covering complexes . . . . . . . . . . 13

2.4.1 Kurosh's theorem for subgroups of free products . . . . . . 14

2.4.2 Graphs of spaces and graphs of groups . . . . . . . . . 16

2.4.3 Normal form theorem revisited . . . . . . . . . . . 18

2.4.4 Subgroups of amalgamated free products and HNN extensions 19

2.4.5 Applications of subgroup theorem . . . . . . . . . . . . 24 
3 Rips Theory $\quad 26$

3.1 Some basic notions . . . . . . . . . . . . . . 26

3.2 Resolving $\mathbb{R}$-trees . . . . . . . . . . . . . . 29

3.2.1 Bands and their unions . . . . . . . . . . . . 29

3.2.2 Dynamical decomposition of unions of bands . . . . . . 31

3.2.3 Bands complexes . . . . . . . . . . . . . 34

3.2.4 A total space decomposition ............. 38

3.2.5 The Kazhdan-Margulis lemma for resolutions of $\mathbb{R}$-trees . . . 38

3.3 The machine . . . . . . . . . . . . . . . . 40

3.3 .1 Moves ....................... 40

3.3.2 Preliminary assumptions and outline . . . . . . . . 4 43

3.3 .3 Process I . . . . . . . . . . . . . . . 44

3.3 .4 Process II . . . . . . . . . . . . . . . 48

3.4 Machine Output . . . . . . . . . . . . . 51

3.4 .1 The surface case ...................... 52

3.4 .2 The toral case . . . . . . . . . . . . 53

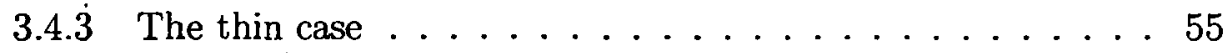

3.5 The decomposition theorem ................ 57

4 Conclusions $\quad 58$

$\begin{array}{lr}\text { References } & 59\end{array}$ 


\section{Introduction}

The main theme of the thesis is the interaction between group theory and topology. In the mathematical literature, often the connections between algebraic properties of finitely generated groups and topological and geometric properties of spaces on which these groups act is the theory of the fundamental group. In the thesis we show several ways in which topological methods help one discover algebraic properties of groups.

First, we consider fundamental groups of graphs of groups. These groups are built from vertex groups and edge groups, according to certain rules, and this information allows one to obtain nice topological spaces associated with $G$ and its subgroups. We show that if a group $G$ is decomposed into a graph of groups, then topological methods provide quite a bit of information about algebraic properties of $G$ and of its finitely generated subgroups. We deal with these topics in Section 2.

Section 3 is dedicated to the Rips theory of isometric groups actions on real trees (or $\mathbb{R}$-trees). It was noticed long ago that the dynamics of group actions on nice spaces reflects algebraic properties of groups. Simplicial trees are one instance of a nice class of spaces. A simplicial tree is an infinite graph without circuits (in the usual graph-theoretical meaning), made into a metric space by giving each edge the unit length. Bass-Serre theory deals with analyzing the algebraic structure of groups acting by automorphisms on simplicial trees. The theory relates group actions on trees with decomposing groups into graphs of groups.

There have been several generalizations of Bass-Serre theory. One is the theory of complexes of groups that provides a higher-dimensional generalization of BassSerre theory. The notion of a graph of groups is replaced by that of a complex of groups, where groups are assigned to each cell in a simplicial complex, together with monomorphisms between these groups corresponding to face inclusions (these monomorphisms are required to satisfy certain compatibility conditions). One can then define an analog of the fundamental group of a graph of groups for a complex of groups.

Another generalization of Bass-Serre theory is Rips theory: real trees include simplicial trees as a particular case. The theory was developed largely in the 1990s, where the work of Eliyahu Rips on the structure theory of stable group actions on $\mathbb{R}$-trees played a key role. This structure theory assigns to a stable isometric action of a finitely presented group $G$ a class of complexes of that action by a stable action of $G$ on a $\mathbb{R}$-tree and hence a decomposition of $G$ in the sense of Bass-Serre theory. Rips theory arises naturally in several contexts in geometric topology: for instance as boundary points of the Teichmuller space, as Gromov-Hausdorff limits of Kleinian group actions, and so on. However, the Rips theory is more than that; it inspires some deep mathematics, for example the use of Rips machine provides substantial shortcuts in modern proofs of Thurston's Hyperbolization Theorem (a certain compactness theorem for sequences of representations). The use of Rips theory is a key tool in the work of Sela on solving the isomorphism problem for (torsion-free) word-hyperbolic groups, Sela's version of the JSJ-decomposition theory and the work of Sela on the Tarski Conjecture for free groups and the theory 
of limit groups. And we still cannot see the end of its further developement, even now a sizable amount of significant results related to it already accumulate in the literature.

\section{Amalgamated Free Products and HNN Exten- sions}

In this section, we assume that a map from a topological space to another topological space is continuous; we also assume that all spaces are path-connected and locally path-connected unless otherwise stated.

We first start with the definitions of amalgamated free products and HNN extensions. Then elaborate Grushko's theorem (Theorem 2.3). Corollary 2.1 says that for any group, we can construct a 2-dimensional $C W$-complex with its fundamental group isomorphic to the given group. By using this, we construct 2-dimensional CW-complexes of a free product of $G_{1}$ and $G_{2} G=G_{1} * G_{2}$ and free group $F$. Throughout the epimorphism $\varphi: F \rightarrow G$, we have the decomposition $F_{1}, F_{2}$ of $F$ such that $\varphi\left(F_{i}\right)=G_{i}$ for $i=1,2$.

The theory of covering spaces and covering complexes is a major tool of topological methods used in combinatorial group theory. We have the Kurosh's subgroup theorem for subgroups of free products (Theorem 2.4). Due to Serre [Ser03], we introduce terminologies of a graph of spaces, a total space and a graph of groups and then re-prove the normal form theorem. See Theorem 2.1, 2.2 and Proposition 2.4. Finally, we have Theorem 2.6 and 2.8 :

Given a fundamental group $G$ of a graph of groups and its subgroup $H, H$ is again a fundamental of groups of a graph of groups, where the vertex groups of $H$ are subgroups of conjugates of the vertex group of $G$ and the edge groups of $H$ are subgroups of conjugates of the edge group of $G$. Moreover, vertices and edges are corresponding to some double cosets.

As applications of Theorem 2.6 and 2.8, we give an example to show that nonfinitely generated groups cannot be expressed as a free product of freely indecomposable subgroups; and prove that any countable group can be embedded in a 2-generator group (Theorem 2.9).

\subsection{Some basic definitions}

Let us recall the basic properties of the fundamental group of a topological space. All details are mainly discussed in [Mas67], [Hat02], and [Mun00].

FACT 2.1 ([Mun00] Theorem 52.1 and 58.3) For any topological space $X$ and $a$ base point $x \in X$, we have a fundamental group of $X$ based at $x$ denoted by $\pi_{1}(X, x)$. A path from $x$ to $y$ induces a group isomorphism $\pi_{1}(X, x) \rightarrow \pi_{1}(X, y)$. A map $f: X \rightarrow Y$ with $f(x)=y$ induces a group homomorphism $\pi_{1}(f): \pi_{1}(X, x) \rightarrow$ 
$\pi_{1}(Y, y)$ where $\pi_{1}(f)$ is a functor of $f$. If $X$ is a deformation retract of $Y$ or vice versa, then $\pi_{1}(X) \cong \pi_{1}(Y)$.

FACT 2.2 Let $p: \tilde{X} \rightarrow X$ be a surjective map. If every point $x$ of $X$ has a neighborhood $U$, and $p^{-1}(U)$ can be written as the union of disjoint open sets $V_{\nu}$ in $\tilde{X}$ for each $\nu$ which the restriction of $p$ to $V_{\nu}$ is a homeomorphism of $V_{\nu}$ onto $U$, then $(\tilde{X}, p)$ is a covering space of $X$ and the following holds :

(1) ([Mas67] Lemma 2.1) If $A$ is a subspace of $X$, and $\tilde{A}$ is a component of $p^{-1}(A)$, then $\left(\tilde{A},\left.p\right|_{\tilde{A}}\right)$ is a covering space of $A$.

(2) ([Mas67] Theorem 4.1) $(\tilde{X}, p)$ induces a monomorphism $\pi_{1}(p): \pi_{1}\left(\tilde{X}, \tilde{x}_{0}\right) \rightarrow$ $\pi_{1}\left(X, p\left(\tilde{x}_{0}\right)\right)$.

(3) ([Mas67) Theorem 5.1) Suppose given a covering map $p:\left(\tilde{X}, \tilde{x}_{0}\right) \rightarrow\left(X, x_{0}\right)$ and a map $f:\left(Y, y_{0}\right) \rightarrow\left(X, x_{0}\right)$. Then a lift $\tilde{f}:\left(Y, y_{0}\right) \rightarrow\left(\tilde{X}, \tilde{x}_{0}\right)$ of $f$ exists if and only if $\pi_{1}(f)\left(\pi_{1}\left(Y, y_{0}\right)\right)$ is a subgroup of $\pi_{1}(p)\left(\pi_{1}\left(\tilde{X}, \tilde{x}_{0}\right)\right)$.

(4) ([Hat02] Proposition 1.34) Given a covering space $(\tilde{X}, p)$ of $X$ and a map $f: Y \rightarrow X$ with two lifts $\tilde{f}_{1}, \tilde{f}_{2}: Y \rightarrow \tilde{X}$ that agree at one point of $Y$, then if $Y$ is connected, these two lifts must agree on all of $Y$.

FACT 2.3 (Seifert-van Kampen's Theorem) Let $X$ be the union of two open subsets $X_{1}$ and $X_{2}$ having intersection $X_{0}$. If $x \in X_{0}$, the images of the two groups $\pi_{1}\left(X_{1}, x\right)$ and $\pi_{1}\left(X_{2}, x\right)$ in $\pi_{1}(X, x)$, under the homomorphisms induced by inclusion, generate the latter group. Now, let $G$ be a group, and let $f_{1}: \pi_{1}\left(X_{1}, x\right) \rightarrow G$ and $f_{2}: \pi_{1}\left(X_{2}, x\right) \rightarrow G$ be homomorphisms. Let $i_{1}, i_{2}, j_{1}, j_{2}$ be the homomorphisms indicated in the following diagram, each induced by inclusion.

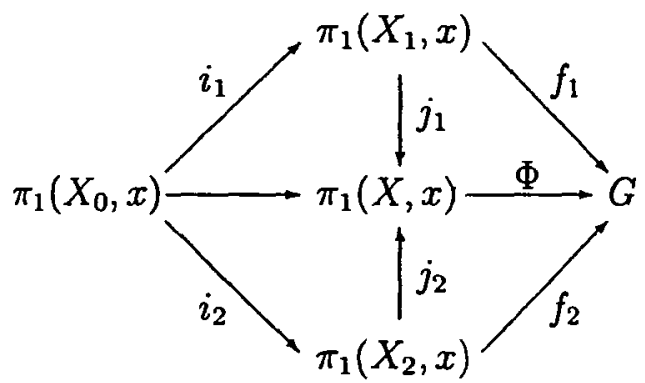

(1) ([Mun00] Theorem 70.1) If $f_{1} \circ i_{1}=f_{2} \circ i_{2}$, then there is a unique homomorphism $\Phi: \pi_{1}(X, x) \rightarrow G$ such that $\Phi \circ j_{1}=f_{1}$ and $\Phi \circ j_{2}=f_{2}$.

(2) ([Mun00] Corollary 70.3)If $X_{0}$ is simply connected, then $\pi_{1}(X, x)$ is the free product of the groups $\pi_{1}\left(X_{1}, x\right)$ and $\pi_{1}\left(X_{2}, x\right)$ with respect to the homomorphisms $j_{1}: \pi_{1}\left(X_{1}, x\right) \rightarrow \pi_{1}(X, x)$ and $j_{2}: \pi_{1}\left(X_{2}, x\right) \rightarrow \pi_{1}(X, x)$.

(3) ([Mun00] Corollary 70.4)If $X_{2}$ is simply connected, then $j_{1}: \pi_{1}\left(X_{1}, x\right) \rightarrow$ $\pi_{1}(X, x)$ is an epimorphism, and its kernel is the smallest normal subgroup of $\pi_{1}\left(X_{1}, x\right)$ containing the image $i_{1}\left(\pi_{1}\left(X_{0}, x\right)\right)$. 
EXAMPLE 2.1 Let $X$ be a space such that $X=A \cup B, A \cap B=\{x\}$, and $A$ and $B$ are each homeomorphic to a circle $S^{1}$. $X$ may be visualized as a curve shaped like a figure "8". See Figure 1.

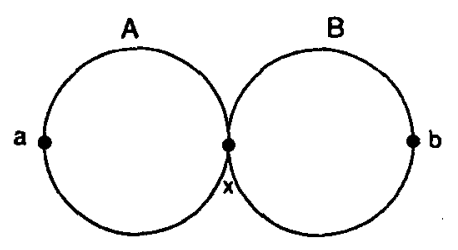

Figure 1: Figure " 8 "

Note that $A$ and $B$ are not open subsets of $X$. However, we should construct two path connected open subsets $X_{1}$ and $X_{2}$ of $X$ and apply Seifert-van Kampen's Theorem (2) above to determine the structure of $\pi_{1}(X, x)$. Choose points a $\in A$ and $b \in B$ such that $a \neq x$ and $b \neq x$. Let $X_{1}=X \backslash\{b\}$, and let $X_{2}=X \backslash\{a\}$. Then $X_{1} \cup X_{2}=X$ and $X_{0}=X_{1} \cap X_{2}=X \backslash\{a, b\}$ is contractible to $x$. Hence, $X_{0}$ is simply connected. Therefore, we can conclude that $\pi_{1}(X, x)=\pi_{1}\left(X_{1}, x\right) * \pi_{1}\left(X_{2}, x\right)$. But $A$ and $B$ are deformation retracts of $X_{1}$ and $X_{2}$, respectively. So $\pi_{1}(A, x) \cong$ $\pi_{1}\left(X_{1}, x\right)$ and $\pi_{1}(B, x) \cong \pi_{1}\left(X_{2}, x\right)$. Thus, $\pi_{1}(X, x) \cong \pi_{1}(A, x) * \pi_{1}(B, x)=\mathbb{Z} * \mathbb{Z}$. Moreover, $\pi_{1}(X, x)$ is a free group on generators $t_{1}, t_{2}$ the classes of the circles.

REMARK 2.1 It follows that $X$ is called the wedge sum $A \vee B$ which is the quotient of the disjoint union $A \sqcup B$ obtained by identifying $a_{0} \in A$ and $b_{0} \in B$ to a single point. More generally one could form the wedge sum $\bigvee_{\nu} X_{\nu}$ of an arbitrary collection of spaces $X_{\nu}$ by starting with the disjoint union $\bigsqcup_{\nu} X_{\nu}$ and identifying points $x_{\nu} \in X_{\nu}$ to a single point. Thus for wedge sum $\bigvee_{\nu} S_{\nu}^{1}$ of circles, $\pi_{1}\left(\bigvee_{\nu} S_{\nu}^{1}\right)$ is a free group, the free product of copies of $\mathbb{Z}$, one for each circle $S_{\nu}^{1}$.

It is true more generally that the fundamental group of any connected graph is free. (The definition of a graph will be introduced when the coming terminology CW-complex is given.)

We now construct a space by the following procedure:

1. Start with a discrete set $X^{0}$, whose points are regarded as 0 -cells.

2. Inductively, form the $n$-skeleton $X^{n}$ from $X^{n-1}$ by attaching $n$-cells $e_{\nu}^{n}$ via maps $f_{\nu}: S^{n-1} \rightarrow X^{n-1}$. This means that $X^{n}$ is the quotient space of the disjoint union $X^{n-1} \bigsqcup_{\nu} D_{\nu}^{n}$ of $X^{n-1}$ with a collection of $n$-disks under the identifications $x \sim f_{\nu}(x)$ for $x \in \partial D_{\nu}^{n}$. ( $D^{n}$ is the unit closed disk or ball in $\mathbb{R}^{n}$ and $\partial D_{\nu}^{n}=S^{n-1}$ the boundary of the n-disk.) Thus as a set, $X^{n}=X^{n-1} \bigsqcup_{\nu} e_{\nu}^{n}$ where each $e_{\nu}^{n}$ is an open $n$-disk.

3. One can either stop this inductive process at a finite stage, setting $X=X^{n}$ for some $n<\infty$, or one can continue infinitely many times, setting $X=$ $\bigcup_{n} X^{n}$. In the latter case $X$ is given the weak topology: A set $A \subset X$ is open (or closed) if and only if $A \cap X^{n}$ is open (or closed) in $X^{n}$ for each $n$. 
DEFINITION 2.1 A space $X$ constructed in this way is called a $C W$-complex or cell complex. If $X=X^{n}$ for some $n$, then $X$ is said to be finite-dimensional, and the smallest such $n$ is the dimension of $X$, the maximum dimension of cells of $X$. A 1-dimensional CW-complex $X=X^{1}$ is what is called a graph. It consists of vertices (the 0-cells) to which edges (the 1-cells) are attached. The two ends of an edge can be attached to the same vertex. A subcomplex of a $C W$-complex $X$ is a closed subspace $A \subset X$ that is a union of cells of $X$. Since $A$ is closed, the map $f_{\nu}^{\prime}: D_{\nu}^{n} \rightarrow X$ of each cell in $A$ has image contained in $A$, and in particular the image of the attaching map $f_{\nu}: S^{n-1} \rightarrow X^{n-1}$ of each cell in $A$ is contained in $A$, so $A$ itself is a $C W$-complex.

The following two facts are assumed with CW-complexes. One is analogous to Seifert-van Kampen's Theorem but with CW-complexes; the other is not true for spaces in general.

FACT 2.4 (Seifert-van Kampen's Theorem for $C W$-complexes [Mas67] Lemma 4.2) Let $X$ be a $C W$-complex, $X_{1}$ and $X_{2}$ be connected subcomplexes of $X$ and let $X=X_{1} \cup X_{2}$. Assume that there exists a non-empty tree $T$ which is a subcomplex of the 1-dimensional $X^{1}$ such that $X_{1} \cap X_{2}=T$. Then, for any vertex $v \in T, \pi_{1}(X, v)=\pi_{1}\left(X_{1}, v\right) * \pi_{1}\left(X_{2}, v\right)$ with respect to the homomorphisms $\pi_{1}\left(j_{i}\right): \pi_{1}\left(X_{i}, v\right) \rightarrow \pi_{1}(X, v)$ induced by the inclusion maps $j_{i}: X_{i} \rightarrow X$ for $i=1,2$.

DEFINITION 2.2 Let $\left(\tilde{X}_{1}, p_{1}\right)$ and $\left(\tilde{X}_{2}, p_{2}\right)$ be covering spaces of $X$. A homomorphism of $\left(\tilde{X}_{1}, p_{1}\right)$ into $\left(\tilde{X}_{2}, p_{2}\right)$ is a continous map $\psi: \tilde{X}_{1} \rightarrow \tilde{X}_{2}$ such that the following diagram is commutative:

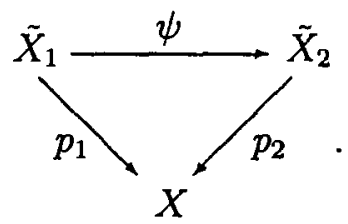

A homomorphism $\psi$ of $\left(\bar{X}_{1}, p_{1}\right)$ into $\left(\tilde{X}_{2}, p_{2}\right)$ is called an isomorphism if there exists a homomorphism $\varphi$ of $\left(\tilde{X}_{2}, p_{2}\right)$ into $\left(\tilde{X}_{1}, p_{1}\right)$ such that both compositions $\varphi \psi$ and $\psi \varphi$ are identity maps. Two covering spaces are said to be isomorphic if there exists an isomorphism of one onto the other. An automorphism is an isomorphism of a covering space onto itself; it may or may not be the identity map.

REMARK 2.2 The composition of two homomorphisms is again a homomorphism, and that, if $(\tilde{X}, p)$ is a covering space of $X$, then the identity map $\tilde{X} \rightarrow \tilde{X}$ is a homomorphism.

Automorphisms of covering spaces are usually called covering transformations or deck transformations. Note that a homomorphism of covering spaces is an isomorphism if and only if it is a homeomorphism in the usual sense. The set of all automorphisms of a covering space $(\bar{X}, p)$ of $X$ is obviously a group under the 
operation of composing maps. We shall use the notation Aut $(\tilde{X})$ to denote this group.

By using covering transformations, we have the following fact :

FACT 2.5 ([CGKZ98] Theorem 1.3.4) Let $X, X^{\prime}$ be $C W$-complexes. For any subgroup $H$ of $\pi_{1}(X, x)$ there is a covering space $\left(X^{\prime}, p: X^{\prime} \rightarrow X\right)$ such that $\pi_{1}(p)\left(\pi_{1}\left(X^{\prime}, x^{\prime}\right)\right)=H$, for $x^{\prime} \in p^{-1}(x)$.

We shall be interested in 2-dimensional $\mathrm{CW}$-complexes, analyzing how the fundamental group is affected by attaching 2-cells. Suppose we attach a collection of 2-cells $e_{\nu}^{2}$ to a space $X$ via maps $f_{\nu}: S^{1} \rightarrow X$, producing a space $Y$. Choose a fixed, closed path $\varphi: I \rightarrow S^{\mathbf{1}}$ which represents an element of the fundamental group $\pi_{1}\left(S^{1},(1,0)\right)$; i.e., the path $\varphi$ goes around the circle $S^{1}$ exactly once. For each $e_{\nu}^{2}$, let $\alpha_{\nu}$ denote the closed path represented by the composed map $f_{\nu} \varphi$. Choose a base point $x \in X$; and for each $e_{\nu}^{2}$, a path class $\beta_{\nu}$ in $X$ with $x$ as initial point and $f_{\nu}(1,0)$ as terminal point. Then, $\gamma_{\nu}=\beta_{\nu} \alpha_{\nu} \beta_{\nu}^{-1}$ is an element of $\pi_{1}(X, x)$.

Proposition 2.1 ([Hat02] Proposition 1.26) The inclusion map $X \hookrightarrow Y$ induces the epimorphism $\pi_{1}(X, x)$ onto $\pi_{1}(Y, x)$, and the kernel $N$ is the smallest normal subgroup containing the set $\left\{\gamma_{\nu}\right\}$. Thus, $\pi_{1}(Y, x) \cong \pi_{1}(X, x) / N$.

Proof. Let us expand $Y$ to a larger space $Z$ that deformation retracts onto $Y$ and is more convenient for applying Seifert-van Kampen's Theorem (3). The space $Z$ is obtained from $Y$ by attaching rectangular strips $S_{\nu}=I \times I$, with $I \times\{0\}$ attached along $\beta_{\nu},\{1\} \times I$ attached along an arc in $e_{\nu}^{2}$, and $\{0\} \times I$ of the different strips identified together. $I \times\{1\}$ of the strips are not attached to anything, and this allows us to deformation retract $Z$ onto $Y$. Thus, $\pi_{1}(Y, x) \cong \pi_{1}(Z, x)$. See Figure 2.

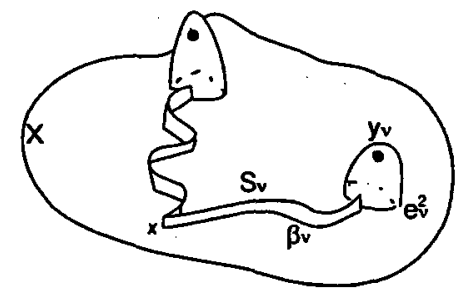

Figure 2: The CW-complex $Z$

In each cell $e_{\nu}^{2}$ choose a point $y_{\nu}$ not in the arc along which $S_{\nu}$ is attached. Let $X_{1}=Z \backslash \bigcup_{\nu}\left\{y_{\nu}\right\}$ and let $X_{2}=Z \backslash X$. Then $X_{1}$ deformation retracts onto $X$, and $X_{2}$ is contractible. So, $\pi_{1}\left(X_{1}, x\right) \cong \pi_{1}(X, x)$ and $X_{2}$ is simply connected. Seifert-van Kampen's Theorem (3) yields that $\pi_{1}(Z, x)$ is isomorphic to the quotient of $\pi_{1}\left(X_{1}, x\right)$ by the normal subgroup generated by $i_{1}\left(\pi_{1}\left(X_{0}=X_{1} \cap X_{2}, x\right)\right)$. Hence, $\pi_{1}(Y, x)$ is isomorphic to the quotient of $\pi_{1}(X, x)$ by the normal subgroup generated by $i_{1}\left(\pi_{1}\left(X_{0}, x\right)\right)$. 
It remains only to show that $\pi_{1}\left(X_{0}, x\right)$ is generated by the loops $\gamma_{\nu}$, or rather by loops in $X_{0}$ homotopic to these loops. Note that $X_{0}=\bigcup_{\nu} A_{\nu}$ where $A_{\nu}=$ $X_{0} \backslash \bigcup_{\mu \neq \nu} e_{\mu}^{2}$ is open, and that $\bigcap_{\nu} A_{\nu}=\{x\}$ which is simple connected. Seifert-van Kampen's Theorem (2) gives that $\pi_{1}\left(X_{0}, x\right) \cong \prod_{\nu} * \pi_{1}\left(A_{\nu}, x\right)$. Since $A_{\nu}$ deformation retracts onto a circle in $e_{\nu}^{2} \backslash\left\{y_{\nu}\right\}$, we obtain $\pi_{1}\left(A_{\nu}, x\right) \cong \mathbb{Z}$ generated by a loop homotopic to $\gamma_{\nu}$, and the result follows.

EXAMPLE 2.2 We compute the fundarnental group of 1-hole torus $Y$. The 1skeleton $X^{1}$ is a wedge sum of two circles with one 0-cell $x$, two 1-cells, and with fundamental group free on two generators $a$ and $b$ by Remark 2.1. $Y$ is a $C W$-complex that is obtained from $X^{1}$ by attaching one 2-cell, as shown in Figure 3. The 2-cell is attached along the loop given by the commutator $[a, b]$. Therefore,
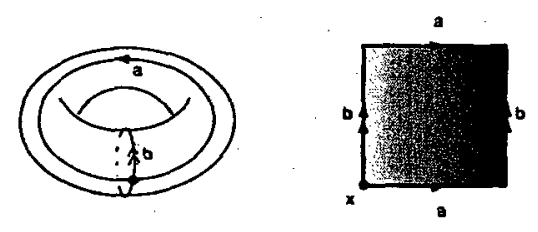

Figure 3: The 1-hole torus

$\pi_{1}(Y, x)=\langle a, b ;[a, b]\rangle$ where $\left\langle s_{i} ; r_{j}\right\rangle$ is called the presentation of $\pi_{1}(Y, x)$ and denotes the group with generators $s_{i}$ and relators $r_{j}$.

Corollary 2.1 ([Hat02] Corollary 1.28) For every group $G$, there is a 2-dimensional $C W$-complex $K$ with $\pi_{1}(K) \cong G$.

Proof. Choose a presentation $G=\left\langle s_{i} ; r_{j}\right\rangle$. This exists since every group is a quotient of a free group, so the $s_{i}$ 's can be taken the generators of this free group with the $r_{j}$ 's generators of the kernel of the map from the free group to $G$. Now construct a CW-complex $K$ from $\bigvee_{i} S_{i}^{1}$ by attaching 2-cells $e_{j}^{2}$ by the loops specified by $r_{j}$.

According to the following proposition, adding cells of dimension $>2$ does not affect $\pi_{1}$, as we see on applying Seifert-van Kampen's Theorem again. So all interest lies in how the 2-cells are attached.

Proposition 2.2 ([Mas67] Theorem 3.1) If the inclusion map of $X$ into $Y$ where $Y$ is obtained from the space $X$ by adjunction of cells of dimension $>2$, then it induces an isomorphism of $\pi_{1}(X, x)$ onto $\pi_{1}(Y, x)$.

FACT 2.6 ([Mas67] Lemma 8.1) Let $\gamma$ be a loop which wraps around $\partial D^{2}$ exactly once. Let $X$ be a space. $A$ map $f: \partial D^{2} \rightarrow X$ can be extended to a map $f^{\prime}: D^{2} \rightarrow$ $X$ if and only if the loop $f \circ \gamma$ is nulhomotopic.

We can use Corollary 2.1 and Fact above to prove the following proposition : 
Proposition 2.3 ([SW79] Lemma 1.5) For any homomorphism $\varphi: \pi_{1}\left(X^{2}, x\right) \rightarrow$ $\pi_{1}(Y, y)$ where $X^{2}$ is a 2-dimensional $C W$-complex and $Y$ is any space, there is a map $\alpha: X^{2} \rightarrow Y$ with $\pi_{1}(\alpha)=\varphi$.

Let's recall some properties of free groups. A rank of a group is the minimal number of generators of that group.

FACT 2.7 Let $F_{m}$ and $F_{n}$ be free groups of rank $m$ and $n$ respectively. Then $F_{m} \cong F_{n}$ if and only if $m=n$.

FACT 2.8 (Universal property of free groups) $G$ is a group generated by $S$. Then $G$ is freely generated by $S$ if and only if $G$ has the following universal property: given a group $H$ and a mapping $\phi: S \rightarrow H$, there is a unique homomorphism $\bar{\phi}: G \rightarrow H$ extending $\phi$.

\subsection{Definitions of amalgamated free products and HNN extensions}

Let $A, B$ and $C$ be groups. Suppose that we have the following pushout diagrams in the category of groups :

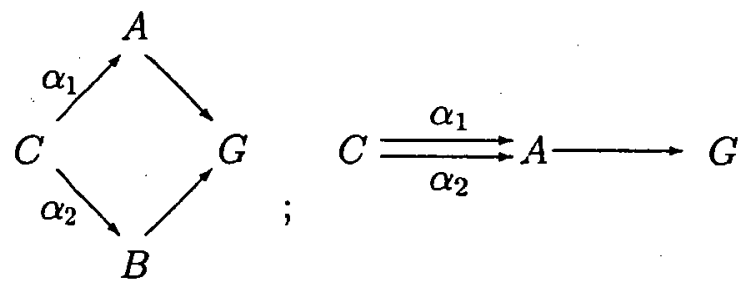

DEFINITION 2.3 If the maps $\alpha_{1}$ and $\alpha_{2}$ are injective, the universal group $G$ is called the free product of $A$ and $B$ amalgamated along $C$, denoted by $A *_{C} B$; for the second case, $G$ is call $H N N$ extension (Higman-Neumann-Neumann) and denoted by $A *_{C}$. If $C$ is the trivial group, then $A *_{C} B$ is denoted by $A * B$ and is called the free product of $A$ and $B$.

Let us recall the traditional combinatorial definitions for $A{ }^{*} C B$ and $A *_{C}$ which are defined by presentation of groups.

$$
A *_{C} B=\left\langle A * B ; \alpha_{1}(c)=\alpha_{2}(c), c \in C\right\rangle ; A *_{C}=\left\langle A, t ; t^{-1} \alpha_{1}(c) t=\alpha_{2}(c), c \in C\right\rangle
$$

where $t$ is called the stable letter. Definition 2.3 is equivalent to the combinatorial one. A sequence of elements $c_{1}, c_{2}, \cdots, c_{n}(n \geq 0)$ is called reduced in $A{ }^{*_{C}} B$ if

1. $c_{i} \in A$ or $B$ for all $i$;

2. $c_{i}, c_{i+1}$ belong to distinct factors; 
3. if $n>1$, then no $c_{i}$ is in $\alpha_{1}(C)$ of $A$ or $\alpha_{2}(C)$ of $B$; and

4. if $n=1$, then $c_{1} \neq 1$.

A sequence of elements $g_{0}, t^{\varepsilon_{1}}, g_{1}, t^{\varepsilon_{2}}, \cdots, t^{\varepsilon_{n}}, g_{n}(n \geq 0)$ is reduced in $A *_{C}$ with $g_{i} \in A$ and $\varepsilon_{i} \in\{ \pm 1\}$ if it has no occurences of $t^{-1}, g_{i}, t$ with $g_{i} \in \alpha_{1}(C)$ and $t, g_{j}, t^{-1}$ with $g_{j} \in \alpha_{2}(C)$ next to each other. A normal form of $A *_{C} B$ is a reduced sequence in $A *_{C} B$. A normal form of $A *_{C}$ is a sequence $g_{0}, t^{\varepsilon_{1}}, g_{1}, t^{\varepsilon_{2}}, \cdots, t^{\varepsilon_{n}}, g_{n}$ $(n \geq 0)$ where

1. $g_{0}$ is an arbitrary element of $A$;

2. if $\varepsilon_{i}=-1$, then $g_{i}$ is a representative of a (right) coset of $\alpha_{1}(C)$ in $A$;

3. if $\varepsilon_{i}=+1$, then $g_{i}$ is a representative of a coset of $\alpha_{2}(C)$ in $A$; and

4. there is no consecutive subsequence $t^{\varepsilon}, 1, t^{-\varepsilon}$.

Theorem 2.1 (The Normal Form Theorem for HNN Extensions) The map $A \rightarrow A *_{C}$ is injective: every element is represented by a unique reduced word.

THEOREM 2.2 (The Normal Form Theorem for Free Products with Amalgamation) The maps $A \rightarrow A *_{C} B, B \rightarrow A *_{C} B$ are injective: every element may be represented by a unique reduced word.

The combinatorial proofs of these two normal form theorems are given by [LS77]. The topological proof of the factors embed are given later.

\subsection{Grushko's theorem}

THEOREM 2.3 (Grushko's theroem) Let $F$ be a finitely generated free groups, let $G_{1}$ and $G_{2}$ be finitely presented groups, let $G=G_{1} * G_{2}$ and let $\varphi: F \rightarrow G$ be an epimorphism. Then there are subgroups $F_{1}$ and $F_{2}$ of $F$ such that $F=F_{1} * F_{2}$ and $\varphi\left(F_{i}\right)=G_{i}$ for $i=1,2$.

To start the proof, we first construct a topological view of the epimorphism $\varphi$ : $F \rightarrow G$.

Consider $G$. Let $X_{i}$ denote a 2-dimensional CW-complex, with a single vertex $x_{i}$ such that $\pi_{1}\left(X_{i}, x_{i}\right) \cong G_{i}$ for $i=1,2$. Such $X_{1}$ and $X_{2}$ exist by Corollary 2.1. Note that $X_{1}$ and $X_{2}$ are pairwise disjoint. Let $v$ be a point not belonging to any of $X_{1}$ or $X_{2}$; join $v$ to the vertex $x_{i}$ by an edge $e_{i}$ for $i=1,2$. Let $X$ denote the union of $X_{1}, X_{2}, e_{1}, e_{2}$, and the vertex $v$; give $X$ the weak topology. Then $X$ is a connected 2-dimensional CW-complex with a base point $v$, and $\pi_{1}(X, v) \cong$ $\pi_{1}\left(X_{1} \cup e_{1} \cup v, v\right) * \pi_{1}\left(X_{2} \cup e_{2} \cup v, v\right)$ by Fact 2.4. Since $\left(X_{i}, x_{i}\right)$ is a deformation retract of $\left(X_{i} \cup e_{i} \cup v, v\right)$ for $i=1,2$, we obtain that $\pi_{1}\left(X_{i}, x_{i}\right) \cong \pi_{1}\left(X_{i} \cup e_{i} \cup v, v\right)$. So, $\pi_{1}(X, v) \cong G_{1} * G_{2}$. 
Consider $F$. Let $\left\{s_{1}, \cdots, s_{n}\right\}$ be a basis for $F$. As we did in Remark 2.1, we have a connected 1-dimensional CW-complex $Y$ with a base point $v_{0}$, and $\pi_{1}\left(Y, v_{0}\right) \cong F$, i.e., the wedge of $n$ circles. Now, consider epimorphism $\varphi: F \rightarrow G$. For each $i=1,2, \cdots, n$, let $\varphi\left(s_{i}\right)$ be written in the normal form in $G=G_{1} * G_{2}$ by Theorem 2.2 , i.e., $\varphi\left(s_{i}\right)=c_{1} c_{2} \cdots c_{k}$ where $c_{j}$ is either in $G_{1}$ or $G_{2}$ and $c_{j}, c_{j+1}$ belong to distinct factors. For each $s_{i}$, divide $S^{1}$ into $k$ segments by $k$ vertices. Denote these segments in order by $W_{1}, \cdots, W_{k}$. For each $s_{i}$, define a map $f_{i}: S^{1} \rightarrow X$ so that $f_{i} \mid w_{j}$ is a closed path in $X_{1}$ or $X_{2}$ representing $c_{j}, j=1, \cdots, k$. Let $K$ denote the union of $Y$ with those new vertices in each $S^{1}$. Therefore, $K$ is a connected 1-dimensional CW-complex with $\pi_{1}\left(K, v_{0}\right) \cong F$ as $\pi_{1}\left(K, v_{0}\right) \cong \pi_{1}\left(X^{1}, v_{0}\right)$.

REMARK 2.3 The maps $f_{i}: S^{1} \rightarrow X$ determine a map $f: K \rightarrow X$ because of the pasting lemma. Thus, $f$ induces the homomorphism $\pi_{1}(f): \pi_{1}\left(K, v_{0}\right) \rightarrow \pi_{1}(X, v)$. We will say that $f$ represents $\varphi$ if there is an isomorphism of $\pi_{1}\left(K, v_{0}\right)$ with $F$ such that the diagram below commutes

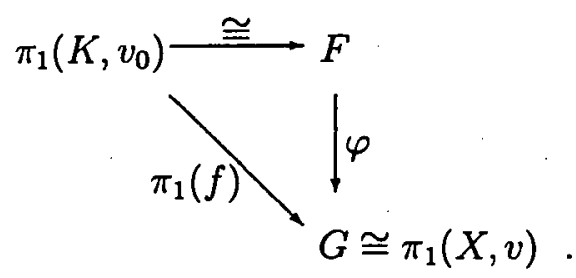

Let $K_{i}$ denote the subcomplex of $K$ consisting of all vertices and all edges $W$ such that $\left.f\right|_{W}$ in $X_{i} \cup e_{i} \cup v$ for $i=1,2$. Note that $K_{1} \cup K_{2}=K$ and $K_{i}$ is not necessarily connected. If both $K_{1}$ and $K_{2}$ were connected and we apply Fact 2.4 , it seems that we could get the result. Unfortunately, $K_{1} \cap K_{2}=Y^{0}$ is the set of all vertices of $K$ which is not a connected set, i.e., $Y^{0}$ is not a tree for now.

In order to apply Fact 2.4, we are going to construct a connected 2-dimensional CW-complex $K^{\prime}$ such that

(1) $K^{\prime}$ contains $K$ as a deformation retract;

(2) a map $f^{\prime}: K^{\prime} \rightarrow X$ extends $f$

(3) $K_{1}^{\prime} \cup K_{2}^{\prime}=K^{\prime}$ where $K_{i}^{\prime}$ is connected containing $K_{i}$ and $f^{\prime}\left(K_{i}^{\prime}\right) \subset X_{i}$ for $i=1,2$ and;

(4) $K_{1}^{\prime} \cap K_{2}^{\prime}$ is a tree.

(1) and (2) imply that $\pi_{1}\left(K^{\prime}, v_{0}\right) \cong F$ and $f^{\prime}$ represents $\varphi$. Applying Fact 2.4, in view of (3) and (4), we have $\pi_{1}\left(K^{\prime}, v_{0}\right)=\pi_{1}\left(K_{1}^{\prime}, v_{0}\right) * \pi_{1}\left(K_{2}^{\prime}, v_{0}\right)$. Take $F_{i}=$ $\pi_{1}\left(K_{i}^{\prime}, v_{0}\right)$ for $i=1,2$. Also, $\pi_{1}\left(f^{\prime}\right)\left(F_{i}\right) \subset G_{i}$ as $f^{\prime}\left(K_{i}^{\prime}\right) \subset X_{i}$; since $f^{\prime}$ represents $\varphi$, we have $\varphi\left(F_{i}\right) \subset G_{i}$. On the other hand, for every $g \in G_{i}$, we have the image of $g$ in $G$ because Theorem 2.2 gives that $G_{i} \rightarrow G$ is injective; we identify $g$ with its the image in $G$; now for every $g$ in $G$, there is $s \in F$ such that $\varphi(s)=g$ because $\varphi: F \rightarrow G$ is surjective; thus we have $\varphi\left(F_{i}\right) \supset G_{i}$. All thogether, we have $\varphi\left(F_{i}\right)=G_{i}$ for $i=1,2$. Therefore, the theorem will be proved. 
Now, the question is whether such a 2-dimensional CW-complex $K^{\prime}$ satisfying properties (1) - (4) always exists. We could use the following lemma to answer this question.

LEMMA 2.1 Let $K$ be a $C W$-complex and $f: K \rightarrow X$ a map representing epimorphism $\varphi: F \rightarrow G$ be as above. Assume that $K_{1}, K_{2} \subset K$ are such that $K=K_{1} \cup K_{2}$ and $K_{1} \cap K_{2}$ is a forest with $\alpha$ components. If $\alpha \geq 2$, then there is a $C W$-complex $K^{1}$ satisfying the following properties:

(1') $K^{1}$ contains $K$ as a deformation retract;

(2') a map $f^{1}: K^{1} \rightarrow X$ extends $f$;

(3') $K_{1}^{1} \cup K_{2}^{1}=K^{1}$ where $K_{i}^{1} \supset K$ and $f^{1}\left(K_{i}^{1}\right) \subset X_{i}$ for $i=1,2$ and;

(4') $K_{1}^{1} \cap K_{2}^{1}$ is a forest with $\alpha-1$ components.

Proof. Choose two distinct components $A$ and $B$ of $K_{1} \cap K_{2}$; let $L$ be a path in $K$ from $A$ to $B$. Note that $\left.f\right|_{A}=\left.f\right|_{B}=v$. Hence, $f \circ L$ is a loop in $X$. As $\pi_{1}(f): \pi_{1}(K) \rightarrow \pi_{1}(X)$ is surjective, there is a loop $\gamma$ in $K$ base at $L(0)$ such that $f \circ \gamma$ is homotopic to the loop $f \circ L$ in $X$. Let $g_{1}$ and $g_{2}$ be elements in $G$ corresponding to $f \circ \gamma$ and $f \circ L$ in $X$. Then $g_{1}^{-1} g_{2}=1$ in $G$. Let $l=\gamma^{-1} L$. Then $l$ is a path in $K$ joining $A$ to $B$ and so $f \circ l$ is a contractible loop in $X$.

We can express $l$ as a union of subpaths $l_{1}, \cdots, l_{n}$ such that the end points of $l_{i}$ lie in $K_{1} \cap K_{2}$ and $f \circ l_{i}$ is a loop in $X_{1}$ or $X_{2}$. Moreover, we can suppose that the maps $f \circ l_{i}$ alternate between $X_{1}$ and $X_{2}$. We say that $l=l_{1} \cdots l_{n}$ has length $n$.

Let $g_{i}$ denote the homotopy class of $f \circ l_{i}$ in $\pi_{1}(X, v)$. Suppose that some $l_{i}$ has the two properties that $g_{i}$ is trivial and that the end points of $l_{i}$ lie in one component of $K_{1} \cap K_{2}$. Then we can change $l$ to $l^{\prime}$ by removing $l_{i}$ and replacing it with a path $l_{i}^{\prime}$ in $K_{1} \cap K_{2}$ which joins the endpoints of $l_{i}$. Clearly, $l^{\prime}$ has length less that $n$. By repeating this process, we can re-arrange that $l$ has no subpaths $l_{i}$ with these two properties.

Now consider $l=l_{1} \cdots l_{r}$ in $K$ and $1=g_{1} \cdots g_{r}$ in $\pi_{1}(X, v)$ where $r \leq n$. Since $\pi_{1}(X, v) \cong G=G_{1} * G_{2}$ and the $g_{i}^{\prime} s$ lie alternately in $G_{1}$ and $G_{2}$, we must have some $g_{i}$ is trivial by Theorem 2.2. The corresponding $l_{i}$ joining distinct components of $K_{1} \cap K_{2}$ and has $f \circ l_{i}$ contractible in $X$.

We now construct a space $K^{1}$ from $K$ by attaching a 1-cell $e^{1}$ to the boundary of $l_{i}$ and then attaching a 2-cell $e^{2}$ to $e^{1} \cup l_{i}$. We would like to extend $f: K \rightarrow X$ to a map $f^{1}: K^{1} \rightarrow X$ by $f^{1}\left(e^{1}\right)=v$ and $f^{1}\left(e^{2}\right)$ in $X_{1}$ or $X_{2}$, however, $f^{1^{-1}}(v)$ does not meet the interior of the 2 -cell $e^{2}$. Note that we have this extension $f^{1}$ because $f \circ l_{i}$ contractible to $v$ in $X$ and the definiton of $f^{1}$ and then by applying Fact 2.6. So, the property (2') is proved. Observe that $K_{1}^{1} \cap K_{2}^{1}=\left(K_{1} \cap K_{2}\right) \cup e^{1}$ which is a forest with $\alpha-1$ components; it is (4'). Clearly, we have (1') and (3').

We can now complete the proof of Grushko's theorem. If $K_{1} \cap K_{2}$ is disconnected, we can apply Lemma above to conclude that there is $K^{1}$ satisfying the properties (1') - (4'). If $K_{1}^{1} \cap K_{2}^{1}$ is not connected, we repeat this process to obtain a new 
CW-complex $K^{2}$, and so on. This process must stop at a finite number of steps, because there were only a finite number of components in $K_{1} \cap K_{2}$. Thus, if $K_{1} \cap K_{2}$ has $n+1$ components, after $n$ steps we obtain a CW-complex $K^{n}$ satisfying all the properties (1) - (4). Let $K^{\prime}=K^{n}, K_{i}^{\prime}=K_{i}^{n}$ for $i=1,2$, and $f^{\prime}=f^{n}$. This proves Grushko's theorem.

We shall illustrate that such a construction is always possible in a particular case.

EXAMPLE 2.3 Let $F=\langle x, y ;-\rangle ;$ and let $G=G_{1} * G_{2}$ where $G_{1}=\left\langle a ; a^{2}=1\right\rangle$ and $G_{2}=\left\langle b ; b^{3}=1\right\rangle$. Define $\varphi: F \rightarrow G$ by $x \mapsto a b a$ and $y \mapsto a b$. As we can check, $\varphi$ is an epimorphism.

The space $K$ in this example is the union of two circles based at $v_{0}$, one divided into two segmetns, and the other divided into three segments. See Figure 4.

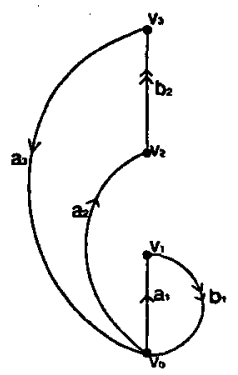

Figure 4: The CW-complex $K$

As we can see that $K_{1}=\left\{a_{1}, a_{2}, a_{3}\right\}$ and $K_{2}=\left\{b_{1}, b_{2}\right\}$. Note that $K_{2}$ is disconnected and $K_{1} \cap K_{2}=\left\{v_{0}, v_{1}, v_{2}, v_{3}\right\}$ which is not a tree yet. Thus, we use Lemma 2.1 to obtain $K^{1}$ first by adjoining the edges $e_{1}^{1}$ and the 2-cells $e_{1}^{2}$. Let $L=a_{1}^{-1} a_{2}$ be a path in $K$ joining two distinct components $v_{1}$ to $v_{2}$. Here, keep in mind that we have to find a path $l$ in $K$ joining $v_{1}$ to $v_{2}$ such that $f \circ l$ is contractible in $X$. Hence, $l_{1}=a_{1}^{-1} a_{2}$ as $a^{-1} a=1$ in $\pi_{1}(X, v)$, attaching $e_{1}^{1}$ to the boundary of $l_{1}$ from $v_{1}$ to $v_{2}$, and attaching $e_{1}^{2}$ to $e_{1}^{1} \cup l_{1}$, as shown in Figure 5.

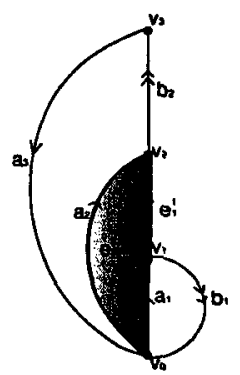

Figure 5: The CW-complex $K^{1}$ 
Now, we obtain that $K_{1}^{1}=\left\{e_{1}^{2}, a_{3}\right\}, K_{2}^{1}=\left\{b_{1}, b_{2}\right\}$ and $K_{1}^{1} \cap K_{2}^{1}=\left\{e_{1}^{1}, v_{0}, v_{3}\right\}$. Repeat this process by using Lemma 2.1. Let $l_{2}=a_{1}^{-1} a_{3}^{-1}$ joining $\left\{v_{1}, v_{2}\right\}$ to $v_{3}$ and $l_{3}=b_{2}^{-1} e_{1}^{1} b_{1}$ joining $\left\{v_{1}, v_{2}, v_{3}\right\}$ to $v_{0}$. Then we obtain the CW-complex $K^{\prime}$, shown in Figure 6, is two pieces that must be glued together along the line $v_{0} v_{3}$ because it is hard to draw two of them in one plane.
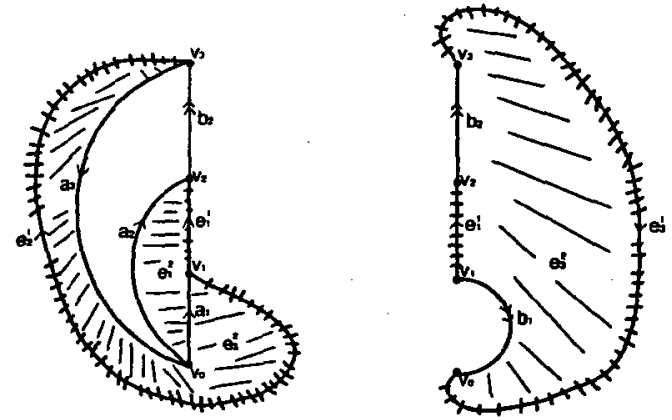

Figure 6: The CW-complex $K^{\prime}$

Hence, we have $K_{1}^{\prime}=\left\{e_{1}^{2}, e_{2}^{2}\right\}$ and $K_{2}^{\prime}=\left\{e_{3}^{2}\right\}$; and $f^{\prime}\left(K_{i}^{\prime}\right)=X_{i}$ for $i=1,2$. Note that $K_{1}^{\prime} \cup K_{2}^{\prime}=K^{\prime}$ and $K_{1}^{\prime} \cap K_{2}^{\prime}=\left\{e_{1}^{1}, e_{2}^{1}, e_{3}^{1}\right\}$ which is a tree. So, by Fact 2.4, we have $\pi_{1}\left(K^{\prime}, v_{0}\right) \cong \pi_{1}\left(K_{1}^{\prime}, v_{0}\right) * \pi_{1}\left(K_{2}^{\prime}, v_{0}\right)$. But from Lemma 2.1, we have that $K$ is a deformation retract $K^{\prime}$; so $\pi_{1}\left(K, v_{0}\right) \cong \pi_{1}\left(K^{\prime}, v_{0}\right)$. All together, $F \cong \pi_{1}\left(K, v_{0}\right) \cong \pi_{1}\left(K_{1}^{\prime}, v_{0}\right) * \pi_{1}\left(K_{2}^{\prime}, v_{0}\right)$.

Grushko's theorem implies a subtle result about generators of $G$.

Corollary 2.2 If $G=G_{1} * G_{2}$, then $r k(G)=r k\left(G_{1}\right)+r k\left(G_{2}\right)$ where $r k(G)$ denotes the rank of $G$.

Proof. If $r=r k(G)$, then there exists a free group $F$ with $r k(F)=r$ and an epimorphism $\varphi: F \rightarrow G$ such that $F=F_{1} * F_{2}$ and $\varphi\left(F_{i}\right)=G_{i}$ for $i=1,2$. Thus, $r k\left(F_{i}\right) \geq r k\left(G_{i}\right)$. Hence, $r \geq r k\left(G_{1}\right)+r k\left(G_{2}\right)$. On the other hand, $G=\left\langle G_{1}, G_{2}\right\rangle$ implies that $r \leq r k\left(G_{1}\right)+r k\left(G_{2}\right)$. Therefore, $r=r k\left(G_{1}\right)+r k\left(G_{2}\right)$.

The following corollary follows from Corollary 2.2 .

COROLlaRY 2.3 If $G$ is a finitely generated group, then $G=\prod_{i=1}^{n} * G_{i}$ for some $n$, where each $G_{i}$ is freely indecomposable (i.e., $G_{i}=A * B$ implies $A$ or $B$ is trivial).

\subsection{Applications of covering complexes}

We use the theory of covering complexes in topological methods to study the algebraic structure of subgroups of free products, amalgamated free products and HNN extension. 


\subsubsection{Kurosh's theorem for subgroups of free products}

Let us consider the special case of amalgamated free product : the free product.

Assume that $G$ is the free product of $G_{1}$ and $G_{2}$. It is natural to ask whether every subgroup $H$ of $G$ is of this type. The well-known theorem of Kurosh uses the theory of covering spaces to describe subgroups of free products.

THEOREM 2.4 (Kurosh's subgroup theorem) If $H$ is a subgroup of $G=G_{1}$ * $G_{2}$, then $H=F *\left(\prod_{\nu} * H_{\nu}\right)$, where $F$ is a free group and $H_{\nu}$ are subgroups of conjugates of $G_{1}$ or $G_{2}$.

Proof. As before, we construct a CW-complex $X$ with fundamental group $G$ by taking 2-dimensional CW-complexes $X_{1}, X_{2}$ with $\pi_{1}\left(X_{i}, x_{i}\right)=G_{i}$ and joining $v$ to $x_{i}$ by an edge $e_{i}$ for $i=1,2$. Then $\pi_{1}(X, v) \cong G_{1} * G_{2}$ by Fact 2.4.

Since $H$ is a subgroup of $G$, there is a covering space $(\tilde{X}, p)$ such that $H=$ $\pi_{1}(p)\left(\pi_{1}(\tilde{X}, \tilde{v})\right)$ for $\tilde{v} \in p^{-1}(v)$ by Fact 2.5. Note that for each $i=1,2, p^{-1}\left(X_{i}\right)=$ $\left\{\tilde{X}_{i j} ; j=1,2, \cdots\right\}$ where $\left(\tilde{X}_{i j},\left.p\right|_{\tilde{X}_{i j}}\right)$ is a covering space of $X_{i}$ by Fact $2.2(1)$. Choose a maximal tree $T_{i j}$ in $\tilde{X}_{i j}$. Let $Y$ denote the union of all the trees $T_{i j}$ together with all the edges $p^{-1}\left(e_{i}\right)$ for all $i$ and $j$. Then $Y$ is a connected 1dimensional CW-complex. Let $T$ be a maximal tree in $Y$ such that $T$ contains each of the $T_{i j}$. Observe that $T$ always exist because we can construct a new graph $Y^{\prime}$ from $Y$ by shrinking each of the trees $T_{i j}$ to a vertex $v_{i j}$; let $q: Y \rightarrow Y^{\prime}$ denote the natural map; choose a maximal tree $T^{\prime}$ in $Y^{\prime}$, and then let $T=q^{-1}\left(T^{\prime}\right)$.

Now, note that $\tilde{X}=Y \cup\left(\bigcup_{i, j}\left(\tilde{X}_{i j} \cup T\right)\right)$ and $Y \cap\left(\bigcup_{i, j}\left(\tilde{X}_{i j} \cup T\right)\right)=T$. Fact 2.4 inductively shows that $\pi_{1}(\tilde{X}, \tilde{v})=\pi_{1}(Y, \tilde{v}) *\left(\prod_{i, j} * \pi_{1}\left(\tilde{X}_{i j} \cup T, \tilde{v}\right)\right)$. But $Y$ is a connected graph. Remark 2.1 tells us that $\pi_{1}(Y, \tilde{v})$ is a free group $F$. Since any tree is contractible, we obtain that $\tilde{X}_{i j}$ is a deformation retract of $\bar{X}_{i j} \cup T$ for each $i, j$. Thus, $\pi_{1}(\tilde{X}, \tilde{v})=F *\left(\prod_{i, j} * \pi_{1}\left(\tilde{X}_{i j}, \tilde{x}_{i j}\right)\right)$ for $\tilde{x}_{i j} \in \tilde{X}_{i j}$.

Let $\tilde{x}_{i j}$ join to $\tilde{v}$ by a path in $\tilde{X}$. We see that $\pi_{1}(p)\left(\pi_{1}\left(\tilde{X}_{i j}, \tilde{v}\right)\right)$ is a conjugate of a subgroup of $G_{1}$ or $G_{2}$. Since $\pi_{1}(p)\left(\pi_{1}(\tilde{X}, \tilde{v})\right)=H$, we have the Kurosh's subgroup result.

Corollary 2.4 If $H$ is a subgroup of a free group, then $H$ is free.

COROLlaRY 2.5 If $H$ is freely indecomposable and not infinite cyclic, and if $H$ is a subgroup of $G_{1} * G_{2}$, then $H$ lies in a conjugate of $G_{1}$ or $G_{2}$.

Proof. By the Kurosh's subgroup theorem, we know that $H=F *\left(\prod_{\nu} * H_{\nu}\right)$. But $H$ is freely indecomposable; so $F$ or $\prod_{\nu} * H_{\nu}$ is trivial. Suppose that $\prod_{\nu} * H_{\nu}$ is trivial. $H=F$ is freely indecomposable implies that $H=F$ is infinite cyclic. It is a contradiction. Hence, $H=\prod_{\nu} * H_{\nu}$. Again, since $H$ is freely indecomposable, we have $H=H_{\nu}$ for some $\nu$. 
LEMmA 2.2 If $G=G_{1} * G_{2}$ and if $w G_{1} w^{-1} \cap G_{i}$ is non-trivial, then $i=1, w \in G_{1}$ and so $w G_{1} w^{-1} \cap G_{i}=G_{1}$.

Proof. Note that $G_{1}$ is non-trivial. Then there is a non-trivial $g \in G_{1}$ such that $w g w^{-1} \in G_{i}$. Without loss of generality, we can write $w=w^{\prime} \alpha$ where $\alpha \in G_{1}$ and $w^{\prime}$ is a reduced word in $G$ ending in $G_{2}$. Thus, $w g w^{-1}=\left(w^{\prime} \alpha\right) g\left(w^{\prime} \alpha\right)^{-1}=$ $w^{\prime}\left(\alpha g \alpha^{-1}\right) w^{\prime-1}=w^{\prime} g^{\prime} w^{\prime-1}$ where $g^{\prime}$ is a non-trivial element in $G_{1}$. But $w^{\prime} g^{\prime} w^{\prime-1} \in$ $G_{i} . w^{\prime} g^{\prime} w^{-1}$ does not lie in $G_{2}$; otherwise, it contradicts to a non-trivial $g^{\prime} \in G_{1}$. Then $w^{\prime} g^{\prime} w^{\prime-1} \in G_{1}$ and so $w^{\prime}$ is a trivial element in $G$. Thus $w \in G_{1}$. Further, $w G_{1} w^{-1} \cap G_{i}=G_{1} \cap G_{i}$ is non-trivial which implies that $i=1$.

THEOREM 2.5 If $G$ is a finitely generated group, then $G=\prod_{i=1}^{n} * G_{i}$ for some $n$, where each $G_{i}$ is freely indecomposable. If also $G=\prod_{i=1}^{n} * G_{i}=\prod_{j=1}^{m} * H_{j}$ where each $G_{i}$ and $H_{j}$ is non-trivial and freely indecomposable, then $m=n$ and, by re-ordering, we have $G_{i} \cong H_{i}$ for each $i$. Further, for each $i$ with $G_{i}$ not infinite cyclic, we have $G_{i}$ conjugate to $H_{i}$.

Proof. The first sentence is just Corollary 2.3 stated again.

Suppose that $G=\prod_{i=1}^{n} * G_{i}=\prod_{j=1}^{m} * H_{j}$ where each $G_{i}$ and $H_{j}$ is non-trivial and freely indecomposable. If each $G_{i}$ is infinite cyclic, then $G$ is a free group with rank $n$ and so each $H_{j}$ is free by Corollary 2.4. As $H_{j}$ is freely indecomposable, it must be infinite cyclic. Then $\prod_{j=1}^{m} * H_{j}$ is a free group with rank $m$. It follows easily by Fact 2.7 that $m=n$. Otherwise, we can re-order the $G_{i}$ 's so that $G_{1}, \cdots, G_{r}$ are not infinite cyclic and $G_{r+1}, \cdots, G_{n}$ are infinite cyclic.

Note that $G_{1}$ is a subgroup of $\prod_{j=1}^{m} * H_{j}$. Corollary 2.5 tells us that $u G_{1} u^{-1} \subset H_{s}$ for some $u \in G$ and some $s \in\{1, \cdots, m\}$. By re-ordering the $H_{j}$ 's, we have $u G_{1} u^{-1} \subset H_{1}$. Thus, $H_{1}$ is not infinite cyclic. Similarly, as $H_{1}$ is a subgroup of $\prod_{i=1}^{n} * G_{i}$, Corollary 2.5 again shows that $v H_{1} v^{-1} \subset G_{t}$ for some $v \in G$ and some $t \in\{1, \cdots, n\}$. Hence, we have $w G_{1} w^{-1} \subset G_{i}$, where $w=v u$. Now by applying Lemma 2.2, we have $t=1$ and $w \in G_{1}$; so $G_{1}=w G_{1} w^{-1} \subset v H_{1} v^{-1} \subset G_{1}$. Therefore, $H_{1}$ is conjugate to $G_{1}$ in $G$. It follows that $G_{1} \cong H_{1}$.

Repeat this process for $G_{2}, \cdots, G_{r}$ to show that $G_{i}$ is conjugate to $H_{i}$, and so $G_{i} \cong H_{i}$ for $i=1, \cdots, r$.

Now consider

$$
G_{r+1} * \cdots * G_{n} \cong G /\left\langle G_{1} * \cdots G_{r}\right\rangle \cong G /\left\langle H_{1} * \cdots H_{r}\right\rangle \cong H_{r+1} * \cdots * H_{m} .
$$

Since $G_{r+1} * \cdots * G_{n}$ is free and $H_{k}$ is a subgroup of $G_{r+1} * \cdots * G_{n}$ for $k \in$ $\{r+1, \cdots, m\}$, we have each $H_{k}$ is free by Corollary 2.4; and since each $H_{k}$ is freely indecomposable, it must be infinite cyclic. Thus, $H_{r+1} * \cdots * H_{m}$ is free. Therefore, by Fact 2.7, we have the rank of $G_{r+1} * \cdots * G_{n}$ is equal to the rank of $H_{r+1} * \cdots * H_{m}$. It follows that $m=n$.

REMARK 2.4 If $G$ is an non-finitely generated group, then the second part of theorem, the uniqueness result clearly still holds for finitely many $m$ and $n$. However, for the first part, the theorem fails. We will see a counter-example in Section 2.4.5. 


\subsubsection{Graphs of spaces and graphs of groups}

We introduce the terminology, due to Serre, of a graph of groups to describe the structure in amalgamated free products.

As we mentioned in the section of Grushko's Theorem, a graph means a 1dimensional CW-complex; however, it doesn't have orientations on these 1-cell edges. In order to avoid these inconvenience, we first introduce the idea of an abstract graph. Basically, it has twice as many edges as a graph.

DEFINITION 2.4 An abstract graph $\Gamma$ consists of a set $V(\Gamma)$, a set $E(\Gamma)$ and two maps $E(\Gamma) \rightarrow V(\Gamma) \times V(\Gamma)$ denoted by $e \mapsto(o(e), t(e))$ and $E(\Gamma) \rightarrow E(\Gamma)$ denoted by $e \mapsto \bar{e}$ which statisfy the following condition: for each $e \in E(\Gamma)$ we have $\overline{\bar{e}}=e, \bar{e} \neq e$ and $o(e)=t(\bar{e})$.

An element $v \in V(\Gamma)$ is called a vertex of $\Gamma$; an element $e \in E(\Gamma)$ is called an (oriented) edge, and $\bar{e}$ is called the inverse edge. The vertex $o(e)=t(\bar{e})$ is called the origin of $e$, and the vertex $t(e)=o(\bar{e})$ is called the terminus of $e$. An orientation of a graph $\Gamma$ is a choice of one edge out of each pair $\{e, \bar{e}\}$.

EXAMPLE 2.4 In practice an abstract graph is often represented by a diagram, using the following convention: a point marked on the diagram corresponds to a vertex of the abstract graph, and a line joining two marked points corresponds to a set of edges of the form $\{e, \bar{e}\}$. For example, the abstract graph $\Gamma_{1}$ having 2 vertices $v_{1}, v_{2}$ and 2 edges $e, \bar{e}$ with $v_{1}=o(e), v_{2}=t(e)$; and the abstract graph $\Gamma_{2}$ having 1 vertex $v$ and 2 edges $e, \bar{e}$ with $v=o(e), v=t(e)$ which are represented by the following diagrams.
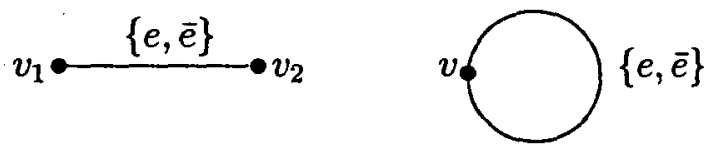

Now we can connect an abstract graph with group theory.

DEFINITION 2.5 A graph of groups $(G, \Gamma)$ consists of an abstract graph $\Gamma$ (which will always be assumed to be connected), a group $G_{v}$ for each $v \in V(\Gamma)$, and a group $G_{e}$ for each $e \in E(\Gamma)$, together with a monomorphism $G_{e} \rightarrow G_{t(e)}$ denoted by $a \mapsto a^{e}$; one requires in addition that $G_{\bar{e}}=G_{e}$.

EXAMPLE 2.5 According to Example 2.4, we then have a graph of groups $\left(G_{1}, \Gamma_{1}\right)$ which consists of three groups $G_{v_{1}}, G_{v_{2}}$ and $G_{e}=G_{\bar{e}}$ and two monomorphisms $G_{e} \rightarrow G_{v_{1}}$ and $G_{e} \rightarrow G_{v_{2}}$ for $\Gamma_{1} ;$ a graph of groups $\left(G_{2}, \Gamma_{2}\right)$ which consists of two groups $G_{v}$ and $G_{e}=G_{\bar{e}}$ and two monomorphisms $G_{e} \rightarrow G_{v}$ for $\Gamma_{2}$. See the following diagrams.

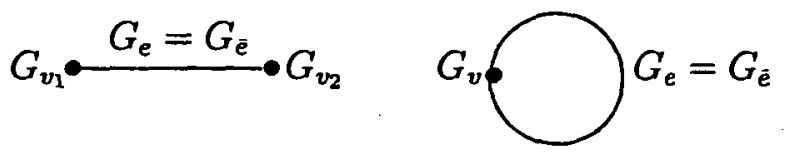


Definition 2.6 A graph of spaces $(X, \Gamma)$ consists of an abstract graph $\Gamma$, a topological space $X_{v}$ for each $v \in V(\Gamma)$, and a topological space $X_{e}$ for each $e \in E(\Gamma)$, with $X_{\bar{e}}=X_{e}$, and an inclusion map $X_{e} \rightarrow X_{t(e)}$.

Given $a(X, \Gamma)$, we can define a total space $X_{\Gamma}$ as the quotient of the union of $\left\{X_{v} ; v \in V(\Gamma)\right\}$ and $\left\{X_{e} \times I ; e \in E(\Gamma)\right\}$ by the equivalence relations defined as follows : $X_{e} \times I \rightarrow X_{\bar{e}} \times I$ by $(x, t) \mapsto(x, 1-t), X_{e} \times\{0\} \rightarrow X_{o(e)}$ by $(x, 0) \mapsto o(e)$ and $X_{e} \times\{1\} \rightarrow X_{t(e)}$ by $(x, 1) \mapsto t(e)$.

REMARK 2.5 A map $f: X \rightarrow Y$ of $C W$-complexes which maps the $n$-skeleton of $X$ into the n-skeleton of $Y$ for all $n$, i.e., $f\left(X^{n}\right) \subset Y^{n}$ for all $n$, is called a cellular map. The cellular approximation theorem says that every $C W$-complexes map is homotopic to a celluar map (See [Hat02] Theorem 4.8). For convenience, we will assume that the spaces are CW-complexes and maps are cellular.

DEFINITION 2.7 The fundamental group of the total space $X_{\Gamma}$ is called the fundamental group $\pi_{1}(G, \Gamma)$ of the graph $(G, \Gamma)$ of groups.

EXAMPLE 2.6 Let us consider the case in Example 2.5. Find' the fundamental groups of the graph of groups.

For the graph of groups $\left(G_{1}, \Gamma_{1}\right)$, let $X_{v_{1}}$ be a 2-dimensional CW-complex with the fundamental group $G_{v_{1}}$, let $X_{v_{2}}$ be a 2-dimensional $C W$-complex with the fundamental group $G_{v_{2}}$, and let $X_{e}=X_{\bar{e}}$ be a 2-dimensional $C W$-complex with the fundamental group $G_{\mathrm{e}}$. Given two monomorphisms $\varphi_{1}: G_{e} \rightarrow G_{v_{1}}$ and $\varphi_{2}: G_{e} \mapsto G_{v_{2}}$, Proposition 2.3 yields that there are two maps $\alpha_{1}: X_{e} \rightarrow X_{v_{1}}$ and $\alpha_{2}: X_{e} \rightarrow X_{v_{2}}$ with $\pi_{1}\left(\alpha_{i}\right)=\varphi_{i}$ for $i=1,2$. Thus, we have a graph of spaces $\left(X_{1}, \Gamma_{1}\right)$. Now we have a total space $X_{\Gamma_{1}}$ by taking $X_{v_{1}}, X_{v_{2}}$ and $X_{e} \times I$, identifing $X_{e} \times I$ with $X_{\bar{e}} \times I$, and gluing $X_{e} \times\{i-1\}$ to $X_{v_{i}}$ using $\alpha_{i}$ for $i=1,2$. Hence, $\pi_{1}\left(X_{\Gamma_{1}}\right)=G_{v_{1}} *_{G_{\mathrm{e}}} G_{v_{2}}$ by Definition 2.3; and so $\pi_{1}\left(G_{1}, \Gamma_{1}\right) \cong G_{v_{1}} *_{G_{e}} G_{v_{2}}$.

Similarly, for the graph of groups $\left(G_{2}, \Gamma_{2}\right)$, take a 2-dimensional CW-complex $X_{v}$ with fundamental group $G_{v}$ and a 2-dimensional $C W$-complex $X_{e}$ with fundamental group $G_{e}$ to form a graph of spaces $\left(X_{2}, \Gamma_{2}\right)$. We obtain a total space $X_{\Gamma_{2}}$ from $X_{v}$ and $X_{e} \times I$ by identifing $X_{e} \times I$ with $X_{\vec{e}} \times I$, and gluing $X_{e} \times\{i\}$ to $X_{v}$ for $i=0,1$. Thus, $\pi_{1}\left(G_{2}, \Gamma_{2}\right) \cong G_{v}^{*} G_{e}$.

REMARK 2.6 The fundamental group $\pi_{1}(G, \Gamma)$ of the graph of groups doesn't depend on the choice of a graph of spaces $(X, \Gamma)$. Given $(G, \Gamma)$, as the procedure stated in the above example, we obtain $(X, \Gamma), X_{\Gamma}$, and $\pi_{1}\left(X_{\Gamma}\right)$. Now we can attach cells of dimension $\geq 3$ to each $X_{v}$ and $X_{e}$ to obtain $C W$-complexes $K_{v}$, $K_{e}$; and note that the map $X_{e} \rightarrow X_{t(e)}$ extends to a map $K_{e} \rightarrow K_{t(e)}$. Thus $(K, \Gamma)$ is a new graph of spaces; its total space $K_{\Gamma}$ is obtained from $X_{\Gamma}$ by adding cells of dimension $\geq 3$. However, $\pi_{1}\left(X_{\Gamma}\right) \cong \pi_{1}\left(K_{\Gamma}\right)$ by Proposition 2.2. So $\pi_{1}(G, \Gamma) \cong \pi_{1}\left(X_{\Gamma}\right) \cong \pi_{1}\left(K_{\Gamma}\right)$. 


\subsubsection{Normal form theorem revisited}

Let's recall the normal form theorems 2.1 and 2.2. We now can use a graph of groups to emphasise the statement of the factors embed and prove it as promised before. We first introduce aspherical space and prove a lemma.

DEFINITION 2.8 An aspherical space is a topological space whose universal cover is contractible.

LEMMA 2.3 If $(K, \Gamma)$ is a graph of aspherical spaces, then the total space $K_{\Gamma}$ is aspherical.

ProOF. Given aspherical spaces $K_{v_{0}}, K_{v_{1}}$ and $K_{e}$, we shall construct the universal covering space $\tilde{K}_{\Gamma} \rightarrow K_{\Gamma}$ and show that $\tilde{K}_{\Gamma}$ is contractible. It will follow then that $K_{\Gamma}$ is aspherical.

Fact $2.2(1)$ implies that $\tilde{K}_{\Gamma}$ is a collection of connected universal covering spaces of $K_{v_{0}}$ and $K_{v_{1}}$ and a collection of connected universal covering spaces of $K_{e} \times I$, by gluing a universal covering space of $K_{e} \times\{i\}$ to a universal covering space $K_{v_{i}}$ for $i=0,1$.

Note that $L_{0}=K_{v_{0}} \cup\left(\bigcup_{o(e)=v_{0}} K_{e} \times I\right)$ is aspherical since $K_{v_{0}}$ is a deformation retract of $L_{0}$, hence the universal covering $\tilde{L}_{0}$ of $L_{0}$ is a deformation retract of the universal covering of $K_{v_{0}}$ and so $\tilde{L}_{0}$ is contractible. Similarly, we have an aspherical space $L_{1}=K_{v_{1}} \cup\left(\bigcup_{t(e)=v_{1}} K_{e} \times I\right)$.

We construct the universal covering space $\tilde{K}_{\Gamma}$ of $K_{\Gamma}$. It will be the union of of an increasing sequence of spaces $\tilde{K}_{0} \subset \tilde{K}_{1} \subset \tilde{K}_{2} \subset \cdots$. Let $\tilde{K}_{0}$ be the universal covering of $L_{0}$. We build $\tilde{K}_{1}$ form $\tilde{K}_{0}$ by attaching the universal covering $\tilde{L}_{1}$ of $L_{1}$, identified along $\tilde{K}_{e} \times I$ for each copy of $\tilde{L}_{1}$. Now we repeat the process to construct $\tilde{K}_{2}$ from $\tilde{K}_{1}$ by attaching $\tilde{K}_{0}$ and identifing along $\tilde{K}_{e} \times I$; and construct $\tilde{K}_{3}$ from $\tilde{K}_{2}$ by attaching the universal covering $\tilde{L}_{1}$ and identifing along $\tilde{K}_{e} \times I$. In the same way, we construct $\tilde{K}_{n+1}$ from $\tilde{K}_{n}$ for $n \geq 0$, and then we set $\tilde{K}_{\Gamma}=\bigcup_{n} \tilde{K}_{n}$.

Note that $\tilde{K}_{n}$ is a deformation retract of $\tilde{K}_{n+1}$ as the construction above. It follows that $\tilde{K}_{\mathrm{r}}$ is contractible since we can deformation retract $\tilde{K}_{n+1}$ onto $\tilde{K}_{n}$, and until we got $\tilde{K}_{0}$ which is contractible to a point.

The natural projection $p: \tilde{K}_{\Gamma} \rightarrow K_{\Gamma}$ clearly gives that $\left(\tilde{K}_{\Gamma}, p\right)$ is the universal covering space of $K_{\Gamma}$. Therefore, $K_{\Gamma}$ is aspherical.

Proposition 2.4 If $(G, \Gamma)$ is a graph of groups, each map $G_{v} \rightarrow \pi_{1}(G, \Gamma)$ is injective.

Proof. Given $(G, \Gamma)$, as in Remark 2.6 we can construct a graph of aspherical spaces $(K, \Gamma)$ and the total space $K_{\Gamma}$. By the previous lemma, we obtain an aspherical total space $K_{\Gamma}$. Inclusion maps $K_{v_{i}} \rightarrow K_{\Gamma}$ for $i=0,1$ induce homomorphisms $\pi_{1}\left(K_{v_{i}}\right) \rightarrow \pi_{1}\left(K_{\Gamma}\right)$. 
We need to show that $\pi_{1}\left(K_{v_{i}}\right) \rightarrow \pi_{1}\left(K_{\Gamma}\right)$ is injective. Suppose that $\gamma$ represents an element of $\pi_{1}\left(K_{v_{i}}\right)$ and is nulhomotopic in $K_{\Gamma}$. We can lift $\gamma$ to $\tilde{\gamma}$ in the universal covering $\tilde{K}_{\Gamma}$ by Fact 2.2 (3). From the construction of previous lemma, $\tilde{\gamma}$ is contained in one of the copies of the universal cover of $K_{v_{i}}$, and so $\bar{\gamma}$ is nulhomotopic in this universal cover. Hence $\gamma$ is nulhomotopic in $K_{v_{i}}$.

\subsubsection{Subgroups of amalgamated free products and HNN extensions}

Let us consider the structure of subgroups of amalgamated free products now.

THEOREM 2.6 If $G=G_{v_{1}} *_{G_{e}} G_{v_{2}}$ or $G_{v} *_{G_{e}}$ and if $H$ is a subgroup of $G$, then $H$ is the fundamental group of a graph of groups, where the vertex groups are subgroups of conjugates of $G_{v_{1}}$ or $G_{v_{2}}$ and the edge groups are subgroups of conjugates of $G_{e}$.

Proof. We construct the total space for $G=G_{v_{1}} *_{G_{e}} G_{v_{2}}$. Let $X_{v_{1}}$ be a 2-dimensional CW-complex with the fundamental group $G_{v_{1}}$, let $X_{v_{2}}$ be a 2dimensional CW-complex with the fundamental group $G_{v_{2}}$, and let $X_{e}=X_{\bar{e}}$ be a 2-dimensional CW-complex with the fundamental group $G_{e}$. Given two monomorphisms $\varphi_{1}: G_{e} \rightarrow G_{v_{1}}$ and $\varphi_{2}: G_{e} \mapsto G_{v_{2}}$, Proposition 2.3 yields that there are two maps $\alpha_{1}: X_{e} \rightarrow X_{v_{1}}$ and $\alpha_{2}: X_{e} \rightarrow X_{v_{2}}$ with $\pi_{1}\left(\alpha_{i}\right)=\varphi_{i}$ for $i=1,2$. Thus, we have a graph of spaces $(X, \Gamma)$. Now we have a total space $X_{\Gamma}$ by taking $X_{v_{1}}$, $X_{v_{2}}$ and $X_{e} \times I$, identifing $X_{e} \times I$ with $X_{\bar{e}} \times I$, and gluing $X_{e} \times\{i-1\}$ to $X_{v_{i}}$ using $\alpha_{i}$ for $i=1,2$. Hence, $\pi_{1}\left(X_{\Gamma}\right)=G_{v_{1}} *_{G_{e}} G_{v_{2}}$ by Definition 2.3.

Since $H$ is a subgroup of $G$, we have a covering space $\left(\tilde{X}_{\Gamma}, p\right)$ of $X_{\Gamma}$ such that $H=\pi_{1}(p)\left(\pi_{1}\left(\tilde{X}_{\Gamma}, *\right)\right)$ by Fact 2.5. Fact 2.2 (1) yields that $\tilde{X}_{\Gamma}$ is constructed from a collection of connected covering spaces of $X_{v_{1}}$ and $X_{v_{2}}$ and a collection of connected covering spaces of $X_{e} \times I$, by gluing a covering space of $X_{e} \times\{i-1\}$ to a covering space $X_{v_{i}}$ for $i=1,2$.

$\bar{X}_{\Gamma}$ looks like a graph $\Gamma$ with a space at each vertex and a space $\times I$ along each edge. If $\Gamma$ was a tree, then $H$ would be a multiple amalgamated free product where each amalgamation is of type $G_{v_{1}} *_{G_{e}} G_{v_{2}}$ but not $G_{v^{*} G_{e}}$. In general, $\Gamma$ is a tree with extra edges attached, and then $H$ is a multiple amalgamated free product together with $\mathrm{HNN}$ extensions, i.e., $H$ is the fundamental group of a graph of groups.

As in the proof of Kurosh's subgroup theorem, fix a base point of $\bar{X}_{\Gamma}$. We then see that every vertex groups or edge groups is a conjugate of some subgroup of $G_{v_{i}}$ for $i=1,2$ or $G_{e}$, respectively.

Similarly, we obtain the statement for $G_{u} *_{G_{e}}$.

REMARK 2.7 One corollary of this result is Kurosh's subgroup theorem. Simiply by setting the edge group $G_{e}$ to trivial, we obtain that the general case $\Gamma$ is a tree $T$ with extra edges attached; and so $H=F *\left(\prod_{\nu} * H_{\nu}\right)$ where $F$ is a free group whose generators correspond to the edges of $\Gamma \backslash T$ and the vertext groups $H_{\nu}$ are subgroups of conjugates of $G_{v_{1}}$ or $G_{v_{2}}$. 
Definition 2.9 A group $G$ splits over a subgroup $G_{e}$ if $G=G_{v^{*} G_{e}}$ or $G=$ $G_{v_{1}} *_{G_{e}} G_{v_{2}}$ with $G_{v_{1}} \neq G_{e} \neq G_{v_{2}}$. If $G$ splits over some subgroup, we say that $G$ is splittable.

EXAMPLE 2.7 1-hole torus group $\langle a, b ;[a, b]\rangle$ is isomorphic to $\langle a\rangle *_{(a)}$. since

$$
\left\langle a *_{\langle a\rangle}=\left\langle a, t ; t^{-1} \alpha_{1}(a) t=\alpha_{2}(a)\right\rangle=\left\langle a, t ; t^{-1} a t=a\right\rangle .\right.
$$

Hence, 1-hole torus group is splittable.

There is another corollary of this theorem which is analogous to Corollary 2.5 in the case of free products.

Corollary 2.6 If $G=G_{v_{1}} *_{G_{e}} G_{v_{2}}$ or $G=G_{v} *_{G_{e}}$ and if $H$ is a finitely generated non-splittable subgroup of $G$, then $H$ lies in a conjugate of $G_{v_{1}}$ or $G_{v_{2}}$, or of $G_{v}$.

Proof. From the theorem, we obtain that $H$ is a fundamental group of graph $(H, \Gamma)$ of groups. As $H$ is finitely generated, there are finitely many generators representing finitely many loops in $\Gamma$; and so there is a finite subgraph $\Gamma^{\prime}$ of $\Gamma$ such that $\pi_{1}\left(H, \Gamma^{\prime}\right)=H$. But $H$ is non-splittable. This implies that one of the vertex groups is equal to $H$. The result follows.

REMARK 2.8 If we consider non-finitely generated non-splittable subgroup $H, H$ may not lie in a conjugate of $G_{v_{1}}$ or $G_{v_{2}}$.

For example, consider $G=\mathbb{Z} *_{\mathbb{Z}}=\left\langle a, t ; t^{-1}\right.$ at $\left.=a^{2}\right\rangle$ and its subgroup $H=$ $\left\langle t^{n} a t^{-n} \forall n \in \mathbb{Z} ; t^{-1} a t=a^{2}\right\rangle$. Note that $H$ is non-finitely generated.

Claim that $H$ is non-splittable.

Indeed, $H$ is isomorphic to dyadic rationals $\left\{\frac{a}{2^{b}} ; a \in \mathbb{Z}, b \in \mathbb{Z}^{+} \cup\{0\}\right\}$ by $t^{n} a t^{-n} \mapsto$ $\frac{1}{2^{|n|}}$ for all $n \in \mathbb{Z}$. Suppose the isomorphism is given that

$$
H=H_{v} *_{H_{e}}=\left\langle S, t^{\prime} ; R, t^{\prime-1} \alpha_{1}\left(H_{e}\right) t^{\prime}=\alpha_{2}\left(H_{e}\right)\right\rangle
$$

where $H_{v}=\langle S ; R\rangle$. For convenience, where we have two injections $\alpha_{1}$ and $\alpha_{2}$ of $H_{e}$ into $H_{v}$, we identify $H_{e}$ with $\alpha_{1}\left(H_{e}\right)$. Then $\alpha_{2}\left(H_{e}\right)=t^{\prime-1} H_{e} t^{\prime}$. Since the image of $t^{\prime}$ in dyadic rationals commutes with all other elements in dyadic rationals, we have $\alpha_{2}\left(H_{e}\right)=H_{e}$ and so $H=\left\langle S, t^{\prime} ; R\right\rangle$. Moreover, $t^{\prime} \in S$ since $t^{\prime} S=S t^{\prime} \in R$. Hence, $H=H_{v}$ and so contradicts to the splittability of $H$. Now suppose that $H=H_{v_{1}} *_{H_{e}} H_{v_{2}}$ with $H_{v_{1}} \neq H_{e} \neq H_{v_{2}}$. For $s_{1} \in H_{v_{1}} \backslash H_{e}$ and $s_{2} \in H_{v_{2}} \backslash H_{e}$, we have $s_{1} s_{2} \neq s_{2} s_{1}$, which contradicts to the images of $s_{1}$ and $s_{2}$ in dyadic rationals commute.

Obviously, $H$ cannot be contained in a conjugate of $\mathbb{Z}$.

Now, let us focus on the covering spaces and fix some notations. Let $X$ be a connected CW-complex based at $x_{0}$ with fundamental group $G$, let $H$ be a subgroup of $G$, and let $(\tilde{X}, p)$ be the corresponding connected covering space (CW-complex) of $X$ based at $\tilde{x}_{0}$ such that $\pi_{1}(p)\left(\pi_{1}\left(\bar{X}, \tilde{x}_{0}\right)\right)=H$ as stated in Fact 2.5 . 
LEMMA 2.4 There is a natural bijection $\bar{\phi}: H \backslash G \rightarrow p^{-1}\left(x_{0}\right)$, where $H \backslash G$ denotes the quotient of $G$ under the action of $H$ by left multiplication.

Proof. Given $g \in G$, let $l$ be a loop in $\left(X, x_{0}\right)$ representing $g$; and let $\tilde{l}$ be a lift of $l$ starting at $\bar{x}_{0}$. Now, define a map $\phi: G \rightarrow p^{-1}\left(x_{0}\right)$ by $g \mapsto \tilde{l}(1)$. This is well-defined because given $g_{1}$ and $g_{2}$ in $G$ with $g_{1}=g_{2}$, we have that the lift $\tilde{l}_{i}$ of a loop $l_{i}$ representing $g_{i}$ for $i=1,2$ are path homotopic and $\tilde{l}_{1}(1)=\tilde{l}_{2}(1)$.

The map $\phi$ is surjective. For given $y \in p^{-1}\left(x_{0}\right)$, there is a path $\tilde{l}$ from $\tilde{x}_{0}$ to $y$ since $\tilde{X}$ is path connected. Thus, $p \circ \tilde{l}$ is a loop in $\left(X, x_{0}\right)$ since $p \circ \tilde{l}(0)=p\left(\tilde{x}_{0}\right)=x_{0}$ and $p \circ \tilde{l}(1)=p(y)=x_{0}$. Since $p \circ \tilde{l}$ represents some $g$ in $G$, we have $\phi(g)=y$.

Using the same notions in the first paragraph, if $\phi\left(g_{1}\right)=\phi\left(g_{2}\right)$, then $\tilde{l}_{1}(1)=\tilde{l}_{2}(1)$ and so $\tilde{l}_{1} \tilde{l}_{2}^{-1}$ is a loop in $\left(\tilde{X}, \tilde{x}_{0}\right)$. Thus, $p \circ\left(\tilde{l}_{1} \tilde{l}_{2}^{-1}\right)$ represents an element $h$ in $H$. Hence, $g_{1} g_{2}^{-1}=h$; so $g_{1}=h g_{2}$. Conversely, if $g_{1}=h g_{2}$, then $\tilde{l}_{1}(1)=\tilde{l}_{2}(1)$ as the lift of a loop representing $h$ is a loop in $\left(\tilde{X}, \tilde{x}_{0}\right)$. Thus, we deduce that $\phi\left(g_{1}\right)=\phi\left(g_{2}\right)$. Therefore, $\phi\left(g_{1}\right)=\phi\left(g_{2}\right)$ if and only if $g_{1}=h g_{2}$ and so $\phi$ induces $\bar{\phi}: H / G \rightarrow p^{-1}\left(x_{0}\right)$ is a bijection.

LEMMA 2.5 If $H$ is a normal subgroup of $G$, then $G / H$ acts on $\tilde{X}$ by automorphisms.

Proof. Let $g \in G$ and let $y \in p^{-1}\left(x_{0}\right)$ be the end point determined by $g$. Then $\pi_{1}(p)\left(\pi_{1}(\tilde{X}, y)\right)=g^{-1} H g=H$ because $H$ is normal in $G$. So, $\pi_{1}(p)\left(\pi_{1}\left(\tilde{X}, \tilde{x}_{0}\right)\right)=$ $\pi_{1}(p)\left(\pi_{1}(\tilde{X}, y)\right)$. Fact $2.2(3)$ and definiton of covering transformations show that there is an automorphism $\psi_{g}:\left(\tilde{X}, \tilde{x}_{0}\right) \rightarrow(\tilde{X}, y)$ such that $\psi_{g}\left(\tilde{x}_{0}\right)=y$.

Define a mapping $\rho: G \rightarrow \operatorname{Aut}(\tilde{X})$ by $g \mapsto \psi_{g}$. We want to show that $\rho$ is a homomorphism of groups. One need only show that $\psi_{g_{1} g_{2}}\left(\tilde{x}_{0}\right)=\psi_{g_{1}} \circ \psi_{g_{2}}\left(\tilde{x}_{0}\right)$ by Fact $2.2(4)$. Let $l_{i}$ be a loop in $\left(X, x_{0}\right)$ representing $g_{i}$ and let $\tilde{l}_{i}$ be a lift of $l_{i}$ in $\left(\tilde{X}, \tilde{x}_{0}\right)$ for $i=1,2$. Thus, there is an automorphism $\psi_{g_{i}}:\left(\tilde{X}, \tilde{x}_{0}\right) \rightarrow\left(\tilde{X}, y_{i}\right)$ such that $\psi_{g_{i}}\left(\tilde{x}_{0}\right)=y_{i}$ for $i=1,2$. Then $l_{1} l_{2}$ lifts to $\tilde{l}_{1}\left(\psi_{g_{1}}\left(\tilde{l}_{2}\right)\right)$, which is a path from $\tilde{x}_{0}$ to $\psi_{g_{1}}\left(y_{2}\right)=\psi_{g_{1}} \circ \psi_{g_{2}}\left(\tilde{x}_{0}\right)$. Therefore, $\rho$ is homomorphism. Note that the kernel of $\rho$ is $H$, so that we have an action of $G / H$ on $\tilde{X}$.

REMARK 2.9 The quotient of $\bar{X}$ by the action of $G / H$ has a natural projection $\pi$ to $X$ and $\pi$ is a covering map. Also, for each $x \in X, \pi^{-1}(x)$ is a single point as $\pi^{-1}\left(x_{0}\right)$ is a single point. Hence, $\pi$ is a homeomorphism.

Now, we can use two lemmas above to learn more about subgroups of free products and subgroups of amalgamated free prodcuts.

Theorem 2.7 If $G=A * B$ where $A, B$ are non-trivial and $H$ is a finitely generated, normal subgroup of $G$, then $H$ is trivial or has finite index in $G$.

Proof. We suppose that $H$ has infinite index in $G$ and will prove that $H$ must be trivial. As Theorem 2.6 described, we know that $H$ is the fundamental group 
of a graph $(H, \Gamma)$ of groups, where the edge groups are trivial and the vertex groups $H_{\nu}$ are subgroups of conjugates of $A$ or $B$. If $T$ is a maximal tree in $\Gamma$, then Remark 2.7 gives that $H=F *\left(\Pi_{\nu} * H_{\nu}\right)$ where $F$ is a free group whose generators correspond to the edges of $\Gamma \backslash T$. $H$ is normal in $G$ yields that $G / H$ acts on $\Gamma$ by Lemma 2.5 .

As $H$ has infinite index in $G$, we have infinitely many representives in $G / H$ that act on $\Gamma$. For any two distinct representatives $g_{1}$ and $g_{2}$ in $G / H$, we have that $g_{1} g_{2}^{-1}$ does not belong in $H$ (otherwise, $\left.g_{1}=g_{2}\right)$, and so $\phi\left(g_{1}\right) \neq \phi\left(g_{2}\right)$ by Lemma 2.4 . Hence, $\Gamma$ has infinitely many edges. But $H=F *\left(\Pi_{\nu} * H_{\nu}\right)$ is finitely generated; we deduce that $\Gamma \backslash T$ is finite and finitely many of finitely generated vertex groups $H_{\nu}$ 's are non-trivial. Hence, $H$ is the fundamental group of some connected finite subgraph $\Gamma^{\prime}$ of $\Gamma$.

Let $E$ be an edge of $\Gamma \backslash \Gamma^{\prime}$. Then removing $E$ from $\Gamma$ gives two subgraphs $\Gamma_{1}$ and $\Gamma_{2}$ and one of these has trivial fundamental group. Thus every edge of $\Gamma$ has this property by the transitive action of $G / H$ on the edges of $\Gamma$. So we do not have $\Gamma \backslash T$ and hence $\Gamma$ is a tree. Note that we must have at most one vertex group can be non-trivial; otherwise, if two or more vertex groups are non-trivial, then removing $E$ which joins one non-trivial vertex group, gives that two subgraphs $\Gamma_{1}$ and $\Gamma_{2}$ with two non-trivial fundamental groups.

Hence, $H$ is contained in a conjugate of $A$ or $B$. Since $H$ is normal in $G$, we have $H$ is contained in the intersection of all conjugates of $A$ or of $B$. But $A \cap b^{-1} A b$ is trivial for any non-trivial element $b \in B$. Therefore, $H$ is trivial.

REMARK 2.10 In the amalgamated free product $G=A *_{C} B$ or $A *_{C}$, it is not necessary that we have an analogous result. For example,

$$
G=\langle a, b ;[a, b]\rangle *_{\langle a=c\rangle}\langle c, d ;[c, d]\rangle
$$

where $H=\langle a\rangle$ is a normal subgroup of $G$; note that $G \cong F_{2} \times \mathbb{Z}$ and so $|G: H|=$ $\infty$.

Let's recall Theorem 2.6. Our next result is to give a more precise structure theorem for subgroups of amalgamated free products.

As before, let $\left(\tilde{X}, \tilde{x}_{0}\right)$ be a connected covering space of $\left(X, x_{0}\right)$ corresponding to $H$ with the projection $p:\left(\tilde{X}, \tilde{x}_{0}\right) \rightarrow\left(X, x_{0}\right)$ where $H=\pi_{1}(p)\left(\pi_{1}\left(\tilde{X}, \tilde{x}_{0}\right)\right)$ is a subgroup of $G=\pi_{1}\left(X, x_{0}\right)$. Let $Y$ be a subcomplex of $X$ which contains $x_{0}$, such that inclusion $j:\left(Y, x_{0}\right) \rightarrow\left(X, x_{0}\right)$ induces a monomorphism $\pi_{1}(j): \pi_{1}\left(Y, x_{0}\right) \rightarrow$ $\pi_{1}\left(X, x_{0}\right)$. We denote the image group $\pi_{1}(j)\left(\pi_{1}\left(Y, x_{0}\right)\right)=A$ and identify $\pi_{1}\left(Y, x_{0}\right)$ with $A$.

LEMMA 2.6 There is a natural bijection $\bar{\theta}$ between the double cosets $H g A$ and the components of $p^{-1}(Y)$ in $\tilde{X}$.

Proof. Let $i$ be the inclusion map of $p^{-1}\left(x_{0}\right)$ into $p^{-1}(Y)$. Recall Lemma 2.4 where $\phi: G \rightarrow p^{-1}\left(x_{0}\right)$. We define $\theta: G \rightarrow p^{-1}(Y)$ by $\theta=i \circ \phi$. 
$\theta$ is surjective. Let $C$ be a component of $p^{-1}(Y)$. Then $\left(C,\left.p\right|_{C}\right)$ is a covering space of $Y$ by Fact $2.2(1)$; and $C$ must contain $\left.y \in p^{-1}\right|_{C}\left(x_{0}\right)$. As $\phi$ is surjective, we obtain that there is $g \in G$ such that $\phi(g)=y$. Thus, $\theta(g)=i(\phi(g))=i(y) \in C$.

Suppose that $\theta\left(g_{1}\right)$ and $\theta\left(g_{2}\right)$ lie in the same component $C$ of $p^{-1}(Y)$. Then $l_{i}$ is a path starting at $\tilde{x}_{0}$ in $C$ representing $g_{i}$ in $\pi_{1}\left(X, x_{0}\right)$ for $i=1,2$. Since $C$ is path connected, $\theta\left(g_{1}\right)$ and $\theta\left(g_{2}\right)$ can be joined a path $l$ in $C$ of $p^{-1}(Y)$. Thus, $p \circ l$ is a loop in $\left(Y, x_{0}\right)$ representing $\alpha \in A$. So, by projecting a loop $l_{1} l l_{2}^{-1}$ of $C$ into $X$, we see that $g_{1} \alpha g_{2}^{-1} \in H$, so that $g_{2}=h g_{1} \alpha$ for some $h \in H$. Conversely, if $g_{2}=h g_{1} \alpha$ for some $h \in H$ and $\alpha \in A$, then lifting to $\bar{X}$ tells us that $\theta\left(g_{1}\right)$ and $\theta\left(g_{2}\right)$ can be joined by a path $l$ in $p^{-1}(Y)$, where $l$ is a lift of $\alpha$.

Therefore, the result follows.

LEMMA 2.7 Let $g \in G, y=\phi(g) \in p^{-1}\left(x_{0}\right)$ and let $C$ be the component of $p^{-1}(Y)$ which contains $y$. Let $\lambda$ be a loop in $X$ representing $g$ and let $l$ be the lift of $\lambda$ which goes from $\tilde{x}_{0}$ to $y$. Then $\pi_{1}(p)\left(\pi_{1}\left(C, \tilde{x}_{0}\right)\right)=H \cap g A g^{-1}$, where we define $\pi_{1}\left(C, \tilde{x}_{0}\right)$ by using the path $l$.

Proof. Since $\pi_{1}(p)\left(\pi_{1}(C, y)\right) \subset \pi_{1}\left(Y, x_{0}\right)=A$, we have $\pi_{1}(p)\left(\pi_{1}\left(C, \tilde{x}_{0}\right)\right) \subset$ $g A g^{-1}$. As $\pi_{1}(p)\left(\pi_{1}\left(C, \tilde{x}_{0}\right)\right) \subset \pi_{1}(p)\left(\pi_{1}\left(\tilde{X}, \tilde{x}_{0}\right)\right)=H$, we obtain $\pi_{1}(p)\left(\pi_{1}\left(C, \tilde{x}_{0}\right)\right) \subset$ $H \cap g A g^{-1}$.

Conversely, let $\beta=g \alpha g^{-1}$ be an element of $H \cap g A g^{-1}$, where $\alpha \in A$. Let $\mu$ be a loop in $Y$ representing $\alpha$. Then $\lambda \mu \lambda^{-1}$ is a loop in $X$ representing $\beta$. Since $\beta \in H$, we have that $\lambda \mu \lambda^{-1}$ lifts to a loop in $\tilde{X}$. Thus, $\lambda$ lifts to $l$ and $\lambda^{-1}$ lifts to $l^{-1}$ and so $\lambda \mu \lambda^{-1}$ lifts to $l m l^{-1}$ where $m$ is some loop in $p^{-1}(Y)$ based at $y$. Hence, $\beta$ lies in $\pi_{1}(p)\left(\pi_{1}\left(C, \tilde{x}_{0}\right)\right)$.

All together, we have shown that $\pi_{1}(p)\left(\pi_{1}\left(C, \tilde{x}_{0}\right)\right)=H \cap g A g^{-1}$ as required.

We now can state a more precise version of the subgroup theorem.

THEOREM 2.8 (Subgroup Theorem) If $H$ is a subgroup of $G=A *_{C}$ (or $\left.A *_{C} B\right)$, then $H$ is the fundamental group of a graph $(H, \Gamma)$ of groups. The vertices of $\Gamma$ correspond to the double cosets $\mathrm{HgA}$ (and $\mathrm{HgB}$ ), and the corresponding groups are $H \cap g \mathrm{Ag}^{-1}$ (and $H \cap g B g^{-1}$ ). The edges of $\Gamma$ correspond to the double cosets $\mathrm{HgC}$ and the corresponding groups are $\mathrm{H} \cap \mathrm{gCg} \mathrm{g}^{-1}$.

Proof. Theorem 2.6 proved that $H$ is the fundamental group of a graph of groups. Previous two lemmas give the rest of results.

REMARK 2.11 In general, if $G$ is any graph of groups, i.e., $G=\prod_{\nu}{ }^{*} C_{\nu} A_{\nu}$, then we have a corresponding result of subgroup theorem for subgroups $H$ of $G$. It follows from the same lemmas.

CoRollaRY 2.7 If $G=G_{1} *_{C} G_{2}$, then either $g G_{1} g^{-1} \cap G_{i}$ is a subgroup of a conjugate of $C$, or $i=1$ and $g \in G_{1}$, so that $g G_{1} g^{-1} \cap G_{i}=G_{1}$. 
Proof. Note that $g G_{1} g^{-1} \cap G_{i}$ is a subgroup of $G$. Let $H=g G_{1} g^{-1} \cap G_{i}$. Then $H$ is the fundamental group of a graph $(H, \Gamma)$ of groups. Let the vertices $v_{1}, v_{2}$ of $\Gamma$ corresponding to the double cosets $H g G_{1}$ and $H G_{i}$. Then they have associated groups $H \cap g G_{1} g^{-1}=H$ and $H \cap G_{i}=H$, respectively.

If $v_{1}$ and $v_{2}$ are distinct vertices of $\Gamma$, choose a path in $\Gamma$ joining them. As each of the groups associated to $v_{1}, v_{2}$, namely $H_{1}, H_{2}$, is an isomorphism with $H$, we see that each vertext and edge of this path has associated group $H$ and they are not identical. Otherwise, if we take any proper subgroup $C$ of $H$, then we have a relation $\alpha_{1}(C)=\alpha_{2}(C)$ in $H_{1} *_{C} H_{2}$ and so $H_{1} *_{C} H_{2}$ is not isomorphic to $H$. Thus, $H$ is the group associated to some edge and so lies in a conjugate of $C$.

If $v_{1}=v_{2}$, then $H g G_{1}=H G_{i}$. This implies $i=1$; otherwise it contradicts to $H g m \neq H n$ if $m \in G_{1} \backslash \alpha_{1}(C)$ and $n \in G_{2} \backslash \alpha_{2}(C)$. Hence, $H g G_{1}=H G_{1}$ and so $g \in G_{1}$ and the result is proved.

Corollary 2.8 If a group $G=A * B, A \neq\{1\} \neq B$, and if $g=\left[g_{1}, g_{2}\right]$ is an non-trivial element of $A$, then $g_{1}$ and $g_{2}$ lie in $A$.

Proof. Let $H$ be the subgroup of $G$ generated by $g_{1}, g_{2}$. The subgroup theorem implies that $H=(H \cap A) * C$, for some subgroup $C$ of $H$. We need to show that $H \subset A$.

Note that $g \in H \cap A$. Suppose that $H$ is not a subset of $A$. Then $C$ is an non-trivial subgroup of $H$. Corollary 2.2 gives that $2=r k(H)=r k(H \cap A)+r k(C)$ and so $H \cap A$ and $C$ are cyclic. Hence the abelianization homomorphism $H \rightarrow H /[H, H]$ injects $H \cap A$ and $C$. Thus the abelian subgroup $H \cap A$ of $H$ implies $g=\left[g_{1}, g_{2}\right]=1$ in $H \cap A$. This contradicts the fact that $g$ is an non-trivial element in $H \cap A$. Hence $H$ must be contained in $A$.

\subsubsection{Applications of subgroup theorem}

Recall Theorem 2.5 and Remark 2.4. We give the promised example of an nonfinitely generated group $G$ which fails to satisfy the first part of Theorem 2.5.

EXAMPLE 2.8 The non-finitely generated group

$$
G=\left\langle a_{0}, a_{1}, a_{2}, \cdots, b_{1}, b_{2}, \cdots ; a_{n-1}=\left[a_{n}, b_{n}\right], \forall n \geq 1\right\rangle
$$

cannot be expressed as a free product of freely indecomposable subgroups.

Proof. First, observe that

$$
G^{\prime}=\left\langle a_{1}, b_{1}\right\rangle *_{C_{1}}\left\langle a_{2}, b_{2}\right\rangle *_{C_{2}} \cdots,
$$

where each factor group is free of rank 2 , each $C_{i}$ is infinite cyclic, the embedding $C_{i} \rightarrow\left\langle a_{i}, b_{i}\right\rangle$ sends a generator to $a_{i}$ and the other embedding $C_{i} \rightarrow\left\langle a_{i+1}, b_{i+1}\right\rangle$ sends the same generator to $\left[a_{i+1}, b_{i+1}\right]$. Clearly, $G^{\prime} \subset G$. But note that each $a_{i}, b_{i}$ lies in $G^{\prime}$ for $i \geq 1$ and that $a_{0}=\left[a_{1}, b_{1}\right] \in G^{\prime}$. Thus, $G=G^{\prime}$ and so $G$ 
can be expressed as an amalgamated free products. Moreover, each $a_{i}$ in $G$ is an non-trivial element as $a_{i}, b_{i}$ is non-trivial for $i \geq 1$ and $a_{0}=\left[a_{1}, b_{1}\right]$ is non-trivial.

Next, let

$$
G^{\prime \prime}=\left\langle a_{1}, a_{2}, \cdots, b_{1}, b_{2}, b_{3}, \cdots ; a_{n-1}=\left\{a_{n}, b_{n}\right\}, \forall n \geq 2\right\rangle .
$$

Clearly, $G^{\prime \prime} \subset G$. However, we observe that each $a_{i}, b_{i}$ lies in $G^{\prime \prime}$ for $i \geq 1$ and that $a_{0}=\left[a_{1}, b_{1}\right] \in G^{\prime \prime}$. Thus, $G=G^{\prime \prime}$. Since

$$
G^{\prime \prime}=\left\langle b_{1}\right\rangle *\left\langle a_{1}, a_{2}, \cdots, b_{2}, b_{3}, \cdots ; a_{n-1}=\left[a_{n}, b_{n}\right], \forall n \geq 2\right\rangle \cong \mathbb{Z} * G,
$$

we have $G \cong \mathbb{Z} * G$. If $G$ can be expressed as a free product of finite number of freely indecomposable factors, it will contradict to the second part of Theorem 2.5. Thus, the only other possibility is that $G$ can be expressed as a free product of infinite number of freely indecomposable factors. However this also is impossible as shown below.

Suppose that $G=G_{1} * G_{2} * \cdots$, where each $G_{i}$ is freely indecomposable and non-trivial. Consider the element $a_{0}$ of $G$. For some $n, a_{0}$ must lie in $G_{1} * \cdots * G_{n}$, which we denote by $A$. Thus, $G=A * B$ with $A$ and $B$ non-trivial and $a_{0} \in A$; and hence $a_{1}, b_{1}$ lie in $A$ by Corollary 2.8. As $a_{1} \in A$, we obtain that $a_{2}$ and $b_{2}$ lie in $A$ as well by the same corollary. By repreating this argument, we see that $G \subset A$. But $A \subset G$ implies $G=A$. Hence, $B$ is trivial, contradicting the hypothesis that $B$ is non-trivial.

We finish this section by proving the famous embedding theorem of Higman and Neumann which states that any countable group can be embedded in a 2-generator group.

THEOREM 2.9 If $G$ is a countable group, then $G$ can be embedded in a 2-generator group.

Proof. Let $x_{1}, x_{2}, \cdots$, be a generating set for $G$. We embed $G$ in $G_{1}=G * \mathbb{Z}$ by the identity mapping. Let $t$ be a generator of $\mathbb{Z}$, and write $y_{0}=t, y_{i}=x_{i} t$. Then $G_{1}$ is generated by $y_{0}, y_{1}, \cdots$ and each $y_{i}$ has infinite order.

Now let $G_{2}=\left\langle G_{1}, t_{0}, t_{1}, \cdots ; t_{i}^{-1} y_{i} t_{i}=y_{i+1}\right\rangle$. Then $G_{2}=\left(\left(G_{1} *_{G_{1}}\right) *_{G_{1}}\right) \cdots$ and hence $G_{1} \subset G_{2}$ by Theorem 2.1. Note that $G_{2}$ is now generated by a set $y_{0}, t_{0}, t_{1}, \cdots$. Since $G_{1}$ is normal in $G_{2}$ and $\left\{t_{0} G_{1}, t_{1} G_{1}, \cdots\right\}$ maps to a free group $F$ by the $t_{i} G_{1}$ 's to a basis for $F$, the map extends to a unique homomorphism $G_{2} / G_{1}$ to $F$. Fact 2.8 gives that $G_{2} / G_{1}$ is freely generated by $t_{0} G_{1}, t_{2} G_{1}, \cdots$. Hence, the subgroup $K$ of $G_{2}$ generated by the $t_{i}$ 's is free and has the $t_{i}$ 's as a basis. We can embed $K$ in a free group of rank two. To see this we observe that if $K_{1}=\left\langle K, b ; b^{-1} t_{i} b=t_{i+1}\right\rangle$, then $K_{1}=K *_{K}=\left\langle t_{0}, b ;-\right\rangle$ and so $K \subset K_{1}$ by Theorem 2.1.

We let $G_{3}=G_{2} *_{K} K_{1}$. Again, $G_{2} \subset G_{3}$ by Theorem 2.1 and $G_{3}$ is generated by $y_{0}, t_{0}$ and $b$ where the embedding $\alpha_{1}$ is the identity mapping and $\alpha_{2}$ maps $t_{0}$ to $t_{0}$ and $t_{i}$ to $b$ for $i \geq 1$. Finally, let $H$ be the subgroup of $G_{3}$ generated by $y_{0}$ and $b$. 
Since $H \cap G_{2}=\left\langle y_{0}\right\rangle$ and $H \cap K_{1}=\langle b\rangle$ are infinite cyclic and $H \cap K$ is trivial, the subgroup theorem gives that $H \supset H \cap G_{2} *_{H \cap K} H \cap K_{1}=\left\langle y_{0}, b ;-\right\rangle$. Conversely, $H \cap g G_{2} g^{-1} \subset H \cap G_{2}, H \cap g K_{1} g^{-1} \subset H \cap K_{1}$ and $H \cap g K g^{-1}$ is trivial for any $g \in G_{3}$; the subgroup theorem yields that $H \subset H \cap G_{2} *_{H \cap K} H \cap K_{1}$. Hence, $H=\left\langle y_{0}, b ;-\right\rangle$, i.e., the subgroup $H$ of $G_{3}$ is free of rank two.

So we can construct $G_{4}=G_{3} *_{H}$ where our two embeddings of $H$ in $G_{3}$ are $\alpha_{1}$ the identity mapping and $\alpha_{2}$ maps $y_{0}$ to $b$ and $b$ to $t_{0}$. Thus, $G_{4}=\left\langle G_{3}, s ; s^{-1} y_{0} s=\right.$ $\left.b, s^{-1} b s=t_{0}\right\rangle$. Then $G_{4}$ is generated by $y_{0}$ and $s$ which completes the proof the embedding theorem.

\section{Rips Theory}

In this section, we study the group actions on an $\mathbb{R}$-trees. Let $T$ be an $\mathbb{R}$-tree, and let $G$ be a finitely presented group acting on $T$. Then this action corresponds to a 2-dimensional CW-complex $Y$ which consists of rectangles called bands that are attached to a graph along the edges called bases. $Y$ is called the union of bands. Proposition 3.3 ensures that $Y$ can be divided into a union of subcomplexes of simplicial and pure type. Further, we could add a finite number of 2-cells to $Y$. Then $Y$ becomes to a band complex $X$. Theorem 3.1 constructs a band complex $X$ which $G$ acts on and is corresponding to the action $G$ on $T$. We say that $X$ resolves the action.

The Rips machine transform each pure component into three types : surface, toral and thin. Each of them carries the important information about the decomposition of $G$. See Proposition 3.9, 3.10 and 3.11.

Theorem 3.2 says that $X$ has a total space decomposition, with the action of the fundamental group of each vertex space on $T$ either pure or trivial, and the action of the fundamental group of each edge space trivial. This theorem completes our main result Theorem 3.4 :

If the action of $G$ on $T$ is pure, then $G$ satisfies one of the following: surface case, $1 \rightarrow K \rightarrow G \rightarrow \pi_{1}(O) \rightarrow 1$, where $K$ is the kernel of the action of $G$ on $T$ and $O$ is $\bar{Y}$ with some 2-cells attached; toral case, $T$ is a line and $1 \rightarrow K \rightarrow G \rightarrow A \rightarrow 1$, where $K$ is the kernel of the action of $G$ on $T$ and $A$ is a subgroup of $\operatorname{Isom}(\mathbb{R})$; thin case, $G$ splits over a subgroup that fixes an arc of $T$.

If the action of $G$ on $T$ is not pure, then $G$ splits (as an amalgamated free product or HNN extension) over a subgroup $E$ which has a short exact sequence $1 \rightarrow K_{E} \rightarrow$ $E \rightarrow C \rightarrow 1$, where $K_{E}$ fixes an arc in $T$ and $C$ is either finite or cyclic. The subgroup $E$ fixes a point in $T$.

\subsection{Some basic notions}

Let $\left(X, d_{X}\right),\left(Y, d_{Y}\right)$ be metric spaces. Recall that a map $h: X \rightarrow Y$ is an isometry if $h$ is surjective and $d_{X}(x, y)=d_{Y}(h(x), h(y))$ for all $x, y$ in $X ;\left(X, d_{X}\right)$ and $\left(Y, d_{Y}\right)$ are isometric if there is an isometry $h: X \rightarrow Y$. A geodesic joining $x$ to $y$ in $X$ is 
a map $\alpha: I \rightarrow X$ from the unit interval $[0,1]$ of $\mathbb{R}$ to $X$ such that $\alpha(0)=x$ and $\alpha(1)=y$ and $d_{X}(\alpha(a), \alpha(b))=|a-b|$ for all $a, b$ in $I ;\left(X, d_{X}\right)$ is called a geodesic metric space if every two points in $X$ are joined by a geodesic.

Definition 3.1 An $\mathbb{R}$-tree is a geodesic metric space in which any two points are joined by a unique arc that is geodesic.

We could think of $\mathbb{R}$-trees as 0-hyperbolic spaces.

EXAMPLE 3.1 Let $X=\mathbb{R}^{2}$ be the set of pairs of real numbers $x=\left(x_{1}, x_{2}\right)$. The SNCF metric $d_{S N C F}(x, y)$ on $X$ is defined as follows. Let $L$ be the straight line passing through $x$ and $O=(0,0)$. If the point $y$ belongs to $L$, then

$$
d_{S N C F}(x, y)=d(x, y)=\sqrt{\left|x_{1}-y_{1}\right|^{2}+\left|x_{2}-y_{2}\right|^{2}} .
$$

If $y$ does not belong to $L$, then

$$
d_{S N C F}(x, y)=d(x, O)+d(O, y) .
$$

Note that $\left(X, d_{S N C F}\right)$ is a geodesic metric space (see [Lec07] Example 18).

We have an $\mathbb{R}$-tree $T$ marked by bold lines in the following figure :

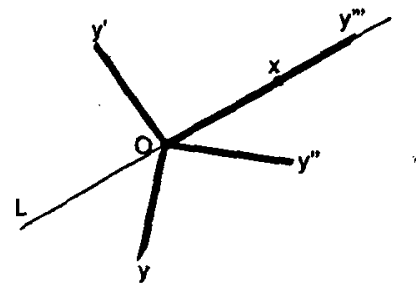

Figure 7: The example of $\mathbb{R}$-tree $T$ in $\left(X, d_{S N C F}\right)$.

DEFINITION 3.2 Let $G$ be a finitely generated group. $A \mathbb{R}$-tree $T$ on which $G$ acts is called a $G$-tree. The action $G$ on $T$ is trivial if there exists a point of $T$ fixed by all of $G$. Otherwise it is non-trivial. A G-tree is minimal if it contains no any proper $G$-invariant substrees.

For any isometry $h$ of a tree $T$, define the translation length of $h$ by

$$
l(h)=\min \{d(x, h(x)) ; x \in T\} .
$$

If $h$ fixes a point in $T$ so that $l(h)=0$, then $h$ is called elliptic; if $l(h)>0$, then $h$ is called hyperbolic. Every hyperbolic element $h$ has a unique axis :

$$
\operatorname{Axis}(h)=\{x \in T ; d(x, h(x))=l(h)>0\} \neq \varnothing,
$$

whereas an elliptic element $h$ has a fixed point set $F i x(h)$. See [Wil04] Lemma 2.2 . 
Proposition 3.1 ([Wil04) Proposition 4.1) If $G$ is a finitely generated group acting non-trivially on an $\mathbb{R}$-tree $T$, then $T$ contains a unique minimal $G$-invariant subtree, which is a countable union of axes.

Proof. We first show that $G$ contains a hyperbolic element. Let $h_{1}, \cdots h_{n}$ be a finite generating set for $G$, and suppose that all $h_{i}$ are elliptic. Then either every pair of generators has intersecting fixed point sets or some pair $h_{i}, h_{j}$ has disjoint fixed point set. In the first case, the action $G$ on $T$ is trivial contradiciting to the non-trivial action. Thus, we only have the second case, in which $h_{i} h_{j}$ is hyperbolic element.

Let $T_{G}$ be the subspace of $T$ defined as the union of the axes of all hyperbolic elements of $G$. Then $T_{G}$ is connected, because if a pair of hyperbolic elements has axes which do not intersect then their product is hyperbolic with an axis intersecting both of theirs.

Finally, $T_{G}$ is itself $G$-invariant, since $h A x i s(\gamma)=\operatorname{Axis}\left(h \gamma h^{-1}\right)$ for hyperbolic $\gamma$ and arbitrary $h \in G$.

If $G$ acts on $T$ and $S$ is a subtree of $T$, denote by $\operatorname{Fix}_{G}(S)$ the subgroup of $G$ whose elements fix $S$ pointwise. A subtree of $T$ is nondegenerate if it is not a point.

DEFINITION $3.3 A$ nondegenerate subtree $S$ of $T$ is stable if for every nondegenerate subtree $S^{\prime}$ of $S$, Fix $_{G}\left(S^{\prime}\right)=F i x_{G}(S)$. A non-trivial action of $G$ on $T$ is called stable if every nondegenerate subtree of $T$ contains a stable subtree.

Proposition 3.2 ([Kap01] Example 12.4) If $T$ is a $G$-tree and $S_{1}, S_{2}$ are stable subtrees with $S_{1} \cap S_{2}$ nondegenerate, then $S_{1} \cup S_{2}$ is stable. In particular, every stable subtree is contained in a unique maximal stable subtree.

Proof. Let $S=S_{1} \cup S_{2}$. Suppose that $S$ is not stable. Then there is $S^{\prime} \subset S$ such that Fix $_{G}\left(S^{\prime}\right) \neq$ Fix $_{G}(S)$. Let $g \in G$ be an element in $F_{i x}\left(S^{\prime}\right) \backslash F i x_{G}(S)$. Without loss of generality, we can assume that $g$ does not lie in $F_{i x}\left(S_{1}\right)$.

Since $S_{1}$ is stable, we conclude that $S_{1} \cap S^{\prime} \subset S_{1}$ is either empty or is a single point. Thus, $S^{\prime} \subset S_{2}$. Since $S_{2}$ is stable, we have $g \in F i x_{G}\left(S_{2}\right)$. Hence, $g \in$ $F_{i x}\left(S_{1} \cap S_{2}\right) \backslash F i x_{G}\left(S_{1}\right)$. However, the stability of $S_{1}$ implies that $S_{1} \cap S_{2}$ is either empty or a single point, which contradicts our assumptions.

LEMMA 3.1 Let $h$ be an isometry of an $\mathbb{R}$-tree $T$, and let $J$ be a nondegenerate arc in $T$. If $h$ induces a nontrivial orientation-preserving partial isometry $h: s \rightarrow s^{\prime}$ for $s, s^{\prime} \subset J \cap A x i s(h)$, then $h$ is hyperbolic and its axis contains $s \cup s^{\prime}$.

Proof. Let $m$ and $n$ be the midpoints of $s$ and $s^{\prime}=h(s)$, respectively. Connect $m, n$ by the geodesic $\alpha \subset J \cap$ Axis $(h)$. Then $h(\alpha) \cap \alpha=\{n\}, h^{-1}(\alpha) \cap \alpha=\{m\}$. Therefore, $\bigcup_{n} h^{n}(\alpha)=\operatorname{Axis}(h)$ is a $h$-invariant geodesic in $T$. 


\subsection{Resolving $\mathbb{R}$-trees}

\subsubsection{Bands and their unions}

DEFINITION $3.4 A$ band $B$ is a product $b \times I$ where $b$ is a closed interval and $I$ is the unit interval.

There is a canonical reflection $\delta_{B}: B \rightarrow B$ given by $\delta_{B}(s, t)=(s, 1-t)$. The closed interval $b$ is identified with $b \times\{0\}$, which is called the bottom of $B$; and $b \times\{1\}$ is called the top of $B$. The closed intervals $b$ and $b^{*}=\delta_{B}(b)$ are called bases of $B$. Note that $b$ and $b^{*}$ are bottom and top of $B$ respectively. The form $\{s\} \times I$ where $s \in b$ is called a vertical fiber, subsets of a vertical fiber are called vertical, and subsets contained in sets of the form $b \times\{t\}$ are called horizontal.

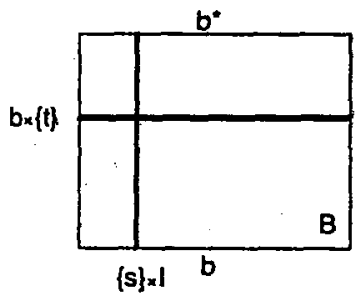

Figure 8: The band $B=b \times I$

EXAMPLE 3.2 The band $B=b \times I$ with two bases $b$ and $b^{*}$ is shown in Figure 8 . Bold lines are horizontal $b \times\{t\}$ and a vertical fiber $\{s\} \times I$.

DEFINITION 3.5 Let $\Gamma$ be a real graph, i.e., a 1-dimensional $C W$-complex $\Gamma$ where each edge is identified with an interval in $\mathbb{R}$ and has an orientation. Let $\mathcal{B}$ be a. finite collection of bands, and for each base $b$ let $f_{b}: b \rightarrow \Gamma$ and $f_{b^{*}}: b^{*} \rightarrow \Gamma$ be isometric embeddings such that the images of the interior of $b$ and $b^{*}$ miss the vertices of $\Gamma$. Then a union of bands is the quotient space

$$
Y=\Gamma \sqcup \coprod_{B \in \mathcal{B}} B
$$

where we identify points of bases with their images in $\Gamma$ under $f_{b}$ and $f_{b^{*}}$. We require that each point of $\Gamma$ belong to the image of at least one base.

EXAMPLE 3.3 Figure 9 gives an example of a union of bands $Y=\Gamma \sqcup B_{1} \sqcup B_{2} \sqcup B_{3}$ where $\Gamma$ is marked by bold lines.

A band $B=b \times I$ is an annulus if $f_{b}=f_{b^{*}} \circ \delta_{B}$. It is a Möbius band if $f_{b}=$ $f_{b^{*}} \circ r_{B} \circ \delta_{B}$, where $r_{B}$ is reflection in the vertical line dividing $B$ in half. See Figure 10.

DEFINITION 3.6 The weight of a base $b$, denoted $w(b)$, is defined as follows: 


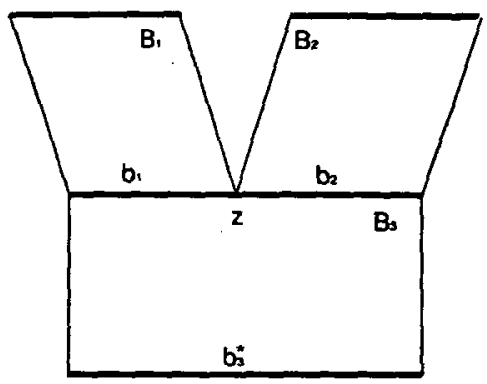

Figure 9: A union of bands $Y$
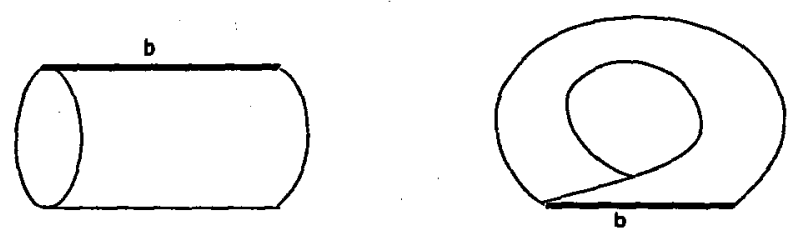

Figure 10: An annulus and a Möbius band

1. if $B$ is an annulus, we let $w(b)=0$;

2. if $B$ is a Möbius band, we let $w(b)=\frac{1}{2}$;

3. otherwise, $w(b)=1$.

The weight of a point $z \in \Gamma$, denoted $w(z)$, is the sum of weights of the bases containing z. A block is the closure of a component of the union of the interiors of bases in $\Gamma$. The complexity $\sigma(\gamma)$ of a block $\gamma$ equals

$$
\max \left\{0,-2+\sum_{b \subset \gamma} w(b)\right\}
$$

The complexity of a union of bands $\sigma(Y)$ is the sum of the complexity of its blocks.

EXAMPLE 3.4 In Example 3.3, $w\left(b_{1}\right)=w\left(b_{2}\right)=w\left(b_{3}\right)=1, w(z)=w\left(b_{1}\right)+$ $w\left(b_{2}\right)+w\left(b_{3}\right)=3$, one of blocks $\gamma$ is $b_{1} \cup b_{2} \cup b_{3}$, and $\sigma(\gamma)=\max \{0,-2+1+1+1\}=$ 1. Note that $\sigma(Y)=1$.

Consider the following figure. $w(z)=\frac{1}{2}+1$ and $\sigma(\gamma)=\max \left\{0,-2+\frac{1}{2}+\frac{1}{2}+1\right\}=0$.

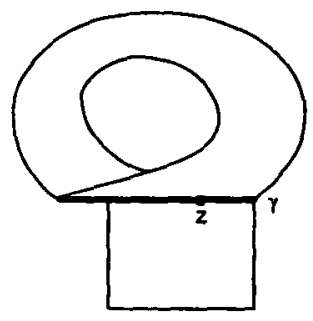

Figure 11: A block $\gamma$ containing two bases. 
A union of bands $Y$ has a natural length metric induced by the metrics on the bands and $\Gamma$. A leaf of $Y$ is an equivalence class generated by the equivalence relation : $x \sim y$ if $x, y$ belong to a vertical fiber of a band. Thus each leaf is a union of vertical fibers in bands. A subset of $Y$ is called vertical if it lies within a single leaf, and horizontal if it is contained in $\Gamma$ or is contained in a single band and is horizontal in that band.

EXAMPLE 3.5 Again, in Example 3.3, we have two leaves $L_{1}$ and $L_{2}$ marked by bold lines in the following figure.

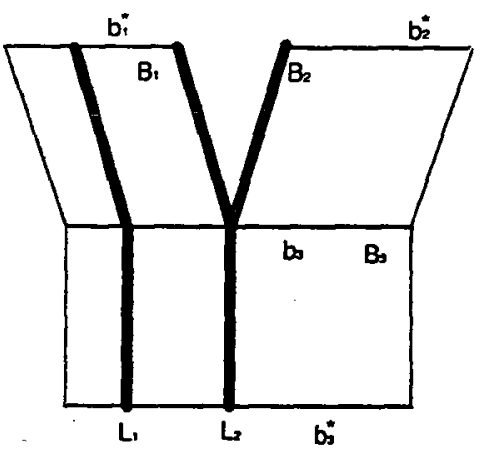

Figure 12: Leaves of $Y$

We can define a transversal measure on a union of bands $Y$. For a band $B$, let $\pi: B \rightarrow b$ be the projection to the first factor. A path $\alpha$ in $B$ is transversal if $\pi \circ \alpha$ is injective; in this case define $\mu(\alpha)$ to be the length of $\pi \circ \alpha$. If the image of $\alpha$ is a vertical of $B$, we let $\mu(\alpha)=0$. Now let $\alpha$ be a path in $Y$. Suppose that we have a finite subdivision of $I$ into subintervals $I_{j}$ such that for each $j$, the restriction $\alpha \mid I_{j}$ is either transversal or vertical in some band. Then we define the transversal measure $\mu(\alpha)=\sum_{j} \mu\left(\alpha \mid I_{j}\right)$.

REMARK 3.1 As above discussion, we conclude that the decomposition of $Y$ into leaves carries a natural transversal measure.

\subsubsection{Dynamical decomposition of unions of bands}

Let $S$ and $S^{\prime}$ in $Y$ be horizontal subsets and let $p$ be a path in a leaf of $Y$.

DEFINITION $3.7 S$ pushes into $S^{\prime}$ along the path $p$ if there is a homotopy $H$ : $S \times I \rightarrow Y$ such that

1. $H(z, 0)=z$ for each $z \in S, H(S, 1) \subset S^{\prime}$;

2. $H(S, t)$ is horizontal for each $t \in I$;

3. $H(z \times I)$ lies in a leaf of $Y$ for each $z \in S$; 
4. there is a point $z_{0} \in S$ such that $H\left(z_{0}, t\right)=p(t), t \in I$.

We say that $S$ pushes onto $S^{\prime}$ along the path $p$ if it pushes into $S^{\prime}$ along the path $p$ and $H(S, 1)=S^{\prime}$. The homotopy $H$ is called pushing.

The horizontal $\varepsilon$-neighbourhood of a point $z \in Y$, denoted $N(z, \varepsilon)$, is the horizontal set of points within $\varepsilon$ of $z$.

DEFINITION 3.8 A subset $Z$ of $Y$ is called pushing-saturated if given a leaf $L$ of $Y$, a path $p: I \rightarrow L$ and $p(0)=z \in Z$ has $N(z, \varepsilon)$ for some $\varepsilon>0$ which pushes along $p$, it follows that $p(1) \in Z$.

Given a point $z \in Y$, define $P(z)$ as the collection of points $y \in Y$ such that $N(z, \varepsilon)$ for some $\varepsilon>0$ pushes onto $N(y, \varepsilon)$ along a path $p: I \rightarrow$ a leaf of $Y$ connects $z$ to $y$. Thus $y \in P(z)$ if and only if $z \in P(y)$. Note that $P(z)$ is the minimal pushing-saturated subset of $Y$ containing $z$. In particular, $Z \subset Y$ is pushing-saturated if and only if $Z \supset P(z)$ for each $z \in z$.

DEFINITION 3.9 A leaf containing a proper pushing-saturated subset is singular; otherwise, it is non-singular. A compact pushing-saturated proper subset of a leaf is called a fault.

EXAMPLE 3.6 The union of bands shown in Example 3.5 has one non-singular leaf $L_{1}$ and one singular leaf $L_{2}$ because it contains a proper pushing-saturated subset $Z_{1}$ from $p(0)=b_{3}^{*} \cap L_{2}$ to $p(1)=b_{3} \cap L_{2}$ where the homotopy is $H(s, t)=$ $(1-t) s+t s$. It has three faults because $L_{2}$ contains three compact proper and pushing-saturated subsets $Z_{1}, Z_{2}$ from $p(0)=b_{3} \cap L_{2}$ to $p(1)=b_{1}^{*} \cap L_{2}$, and $Z_{3}$ from $p(0)=b_{3} \cap L_{2}$ to $p(1)=b_{2}^{*} \cap L_{2}$.

Note that each leaf of $Y$ is pushing-saturated. We give a necessary condition for singularity of a leaf $L$ of $Y$, i.e., for a leaf $L$ of $Y$ to contain a proper pushingsaturated subset (see Figure 13): Either

1. the leaf $L$ contains a cut point there is a point $z \in L \cap \Gamma$ that locally separates the union of $\Gamma$ and those bands that contain $z$, or

2. the leaf $L$ contains a dead end, i.e., a point $z \in L \cap \Gamma$ such that $z$ is an endpoint of a base $b$ and an interior point of a base $b^{\prime}$.

Cut points or dead ends are called critical points of $L$. Hence, each singular leaf contains a critical point.

DEFINITION 3.10 A union of bands $Y$ is simplicial if every leaf of $Y$ is compact; it is minimal if every pushing-saturated subset is dense in $Y$. 

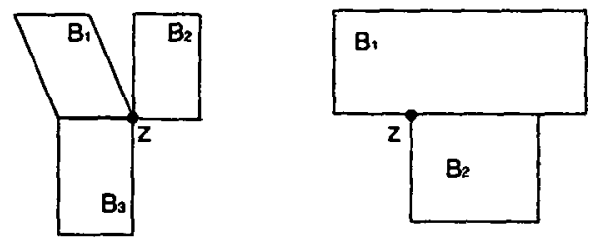

Figure 13: Critical points of leaves

Let $L$ be a compact non-singular leaf in $Y$. Then all nearby leaves are also compact and non-singular. Let $U(L)$ be the maximal connected neighbourhood of $L$ consisting of compact non-singular leaves. We call that $U(L)$ is a simiplicial component of $Y$. The boundary of $U(L)$ must consist of singular leaves, which contain a fault. Let $U$ be the union of all such $U(L)$. Choose a maximal connected $M$ of $Y \backslash U$. Then $M$ is not simplicial and is called a minimal component of $Y$. The reason to define like this becomes clear when the following proposition is proven.

Proposition 3.3 Each union of bands contains only finitely many singular leaves and finitely many faults. Suppose $Y$ contains no faults at all. Then $Y$ splits into a disjoint union of components

(1) each component of $Y$ is either minimal or simplicial;

(2) each simplicial component is an interval bundle over a leaf.

Proof. Since critical points only appear in the corners of bands, and $Y$ only has finitely many corners as it has finitely many bands, we have that there are only finitely many critical points. Since each singular leaf contains a critical point, there are finitely many singular leaves. On the other hand, since a fault is a compact proper pushing-saturated subset of a leaf, it is contained in a compact singular leaf. Hence, $Y$ has finitely many faults as we only have finitely many singular leaves.

Let $L$ be a compact non-singualr leaf, and let $U(L)$ a the simplicial component of $Y$. Then clearly it is an interval bundle over $L$. This proves (2).

Now, we need to prove (1). Let $M$ be a component of $Y \backslash U$, and let $Z$ be a pushing-saturated subset of $M$. We need to show that $Z$ is dense in $M$. Without loss of generality, we may assume that $Z$ is contained in an infinitely long leaf because of the non-compact property.

Step 1 : We claim that there is a number $\delta>0$ depending on $Z$ such that for every base $b$, every component in $b \backslash \bar{Z}$ has length at least $\delta$, where $\bar{Z}$ is the closure of $Z$.

Since $Z$ is an infinitely vertical pushing-saturated subset of $M$ and $Y$ only has finitely many bands, $Z$ has to intersect some band infinitely many times. Without loss of generality, we can assume that $Z \cap b$ is an infinite set. Choose $\delta$ such that for $x$ an end-point of a base $c, d\left(x, c^{\circ} \cap Z\right)=0$ or $\geq \delta$.

We prove this claim by contradiction. Suppose that $J$ is a component of $b \backslash \bar{Z}$ that has length $\lambda<\delta$. Since the accumulation set of a pushing-saturated set is 
again pushing-saturated, we conclude that for $x$ an end-point of $J$, the set $P(x)$ has infinitely long vertical set intersecting $b$ infinitely many times. So, there exists $y, z \in P(x) \cap b$ that satisfy the following :

1. $d(y, z)<\lambda$

2. a nondegenerate subinterval $[x, u]$ of $J$ pushes into the interval $[y, z]$ along a vertical path $p$ connecting $x$ to $y$.

Let $x^{\prime} \in J$ be such that $d\left(x, x^{\prime}\right)=\lambda$. Since $x^{\prime}$ is not contained in $\bar{Z}$, the interval $\left[x, x^{\prime}\right]$ does not push into $[y, z]$ along $p$. The path $p$ is a finite composition of vertical paths $p_{1} * \cdots * p_{k}$, each contained in a single band. Hence for some $j \geq 1$, $\left[x, x^{\prime}\right]$ pushes along $p^{\prime \prime}=p_{1} * \cdots * p_{j-1}$ but does not push along $p^{\prime}=p_{1} * \cdots * p_{j}$. (If $j=1$, then we let $p^{\prime \prime}$ be the constant path.)

1. The point $p^{\prime \prime}\left(x^{\prime}\right)$ arrived at by pushing $x^{\prime}$ along $p^{\prime \prime}$ is not in $\bar{Z}$; if it was, the interval $\left[p^{\prime \prime}(x), p^{\prime \prime}\left(x^{\prime}\right)\right]$ could be pushed into $[y, z]$, where $p^{\prime \prime}(x)$ is a point by pushing $x$ along $p^{\prime \prime}$.

2. $p^{\prime \prime}\left(x^{\prime}\right)$ is an end-point of a base $c$; otherwise, it could be continuously pushed along $p^{\prime}$.

3. $0<d\left(p^{\prime \prime}\left(x^{\prime}\right), p^{\prime \prime}(x)\right)=\lambda<\delta$ since the entire open interval $\left(x, x^{\prime}\right)$ pushes along $p^{\prime \prime}$ and so its image is disjoint from $\bar{Z}$.

This contradicts our choice of $\delta$ and hence proves the claim.

Step 2 : Now consider $b \cap \bar{Z}$ for a base $b$ in $M$. Let $x$ be a point of the boundary of $b \cap \bar{Z}$ in $b$ which is not an end-point of $b$, and let $K=P(x)$ be a pushing-saturated subset. Suppose that $J$ in $b$ is an interval with end-point $x$ such that $J^{\circ} \cap \bar{Z}=\varnothing$. Choose $\delta$ depending on $K$ given by the previous claim, and let $y, z \in K$ be such that :

1. $0<d(y, z)<\delta$;

2. if $p$ is a path in $K$ connecting $x$ to $y$, then $p$ pushes a nondegenerate subinterval $\left[x, x^{\prime}\right]$ in $J$ into the interval $[y, z]$.

Then $[y, z] \subset \bar{K} \subset \bar{Z}$. However, $\left[x, x^{\prime}\right]$ pushes into $[y, z]$ and $\left(x, x^{\prime}\right] \cap \bar{Z}=\varnothing$. Hence $Z$ is dense in $M$.

\subsubsection{Bands complexes}

Observe that a union of bands $Y$ is homotopic to a finite connected graph $\Gamma$ by collapsing the bands so $\pi_{1}(Y) \cong \pi_{1}(\Gamma)$ is a free group. Thus unions of bands do not perfectly resolve $\mathbb{R}$-trees as desired. To get more complicated finitely presented groups, we have to add 2-cells to the unions of bands. 
Definition $3.11 A$ band complex $X$ is a finite 2-dimensional $C W$-complex based on a union of bands $Y$ obtained by taking $Y$ and attaching 0-, 1-, and 2-cells as follows :

1. the 1-cells of $X$ meet $Y$ in a subset of $\Gamma$;

2. each component of the intersection of a 2-cell with $\Gamma$ is a point;

3. each component of the intersection of a 2-cell with a band is vertical.

EXAMPLE 3.7 We first take a union of bands $Y$ from Example 3.3 to form a band complex $X$. A 1-cell is in bold and one 2-cell is shaded.

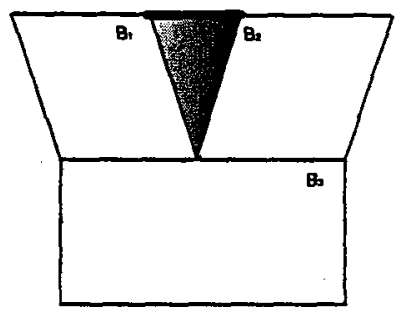

Figure 14: A band complex for Example 3.3

Another example of a band complex for a simplex. The real graph $\Gamma$ is in bold, the union of bands $Y=\Gamma \sqcup B_{1} \sqcup B_{2} \sqcup B_{3}$ and the 2-cells are shaded.

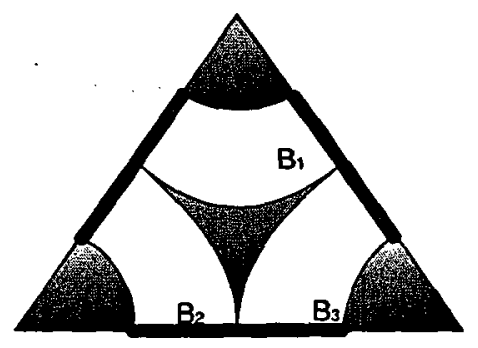

Figure 15: A band complex for simplex

We define the leaves in a band complex $X$ based on a union of bands $Y$ as the leaves in $Y . X$ is minimal or simplicial if $Y$ is. The complexity $\sigma(X)$ is defined as $\sigma(Y)$.

A path $\alpha: I \rightarrow X$ is said to be transversal if $I$ can be divided into finitely many subintervals $I_{j}$ such that for each $j$, either

1. $\alpha \mid I_{j}: I_{j} \rightarrow Y$ is transversal or vertical in $Y$, in which case we let $\mu\left(\alpha \mid I_{j}\right)$ be the transversal measure of $\alpha \mid I_{j}$ as defined before, or

2. $\alpha\left(I_{j}\right)$ is contained in the closure of $X \backslash Y$, in which case $\mu\left(\alpha \mid I_{j}\right)=0$. 
This defines the transversal measure on $X$.

Let $G$ be a group and let $A$ and $B$ be two associated $G$-sets. A mapping $f: A \rightarrow B$ is said to be $G$-equivariant if $f(g \cdot a)=g \cdot f(a)$ for all $g \in G$ and all $a \in A$.

DEFINITION 3.12 A resolution of an action of a finitely presented group $G$ on an $\mathbb{R}$-tree $T$ is a band complex $X$ with $\pi_{1}(X) \cong G$ and a $G$-equivariant map $f: \tilde{X} \rightarrow T$ satisfying the following conditions :

1. for each leaf $L$ in $X$ and each component $\tilde{L}_{0}$ of $L$ 's lift to $\tilde{X}$, the image $f\left(\tilde{L}_{0}\right)$ is a point;

2. each base $b$ in $X$ and each component $\tilde{b}_{0}$ of $b$ 's lift to $\bar{X}, \tilde{b}_{0}$ can be subdivided into finitely many subintervals $I_{j}$ such that $f \mid I_{j}$ is an isometric embedding for each $j$.

THEOREM 3.1 If a finitely presented group $G$ acts on an $\mathbb{R}$-tree then a resolution exists.

ProOF. Note that there exists a 2-dimensional CW-complex $X$ with $\pi_{1}(X) \cong G$ by Corollary 2.1. Construct a band complex structure for $X$ and a $G$-equivariant mapping $f: \tilde{X} \rightarrow T$ as follows.

Define $f$ on the 0 -skeleton of $\tilde{X}$ to be $G$-equivariant. Extend it to the 1 -skeleton by requiring that it is constant on small neighbourhoods of vertices and isometrically embeds on the rest of the edges. Finally extend it to the interior of the 2 -skeleton in the natural manner. The case of Figure 15 illustrates that the three vertices have distinct images in $T$. Note that if all three vertices have the same image, the whole simplex becomes a 2-cell.

EXAMPLE 3.8 Let $G=\langle a, b ;[a, b]\rangle$ act on an real line $T$ with the usual metric such that $a \cdot 0=x, b \cdot 0=y$ and $a b \cdot 0=b a \cdot 0=z$. Construct $a$ resolving band complex $X$.

We first construct the 2 dimensional $C W$-complex $X$ with the fundamental group $G$; and then construct the covering complex $(\bar{X}, p)$ of $X$. Now choose a $G$ equivariant map $f$ from the vertices of $\tilde{X}$ to $T$, for instance, from $f^{-1}(0), f^{-1}(x)$, $f^{-1}(y)$ and $f^{-1}(z)$ to $0, x, y$ and $z$, respectively. Thus, for each edge $\left[f^{-1}(0), f^{-1}(x)\right]$, $\left[f^{-1}(0), f^{-1}(y)\right]$ and $\left[f^{-1}(0), f^{-1}(z)\right]$ of $\tilde{X}$, there is a unique arc $[0, x],[0, y]$ and $[0, z]$ in $T$, respectively. Extend $f$ to a map from $\left[f^{-1}(0), f^{-1}(x)\right],\left[f^{-1}(0), f^{-1}(y)\right]$ and $\left[f^{-1}(0), f^{-1}(z)\right]$ to $[0, x],[0, y]$ and $[0, z]$ that is constant in a neighborhood of the vertices and monotone off this neighborhood. See the following figure 16.

REMARK 3.2 The resolving band complex $X$ constructed in theorem above is always such that $Y$ is connected and $\pi_{1}(Y) \rightarrow \pi_{1}(X)$ is surjective. However, in general, the resolving band complex $X$ is not minimal. 


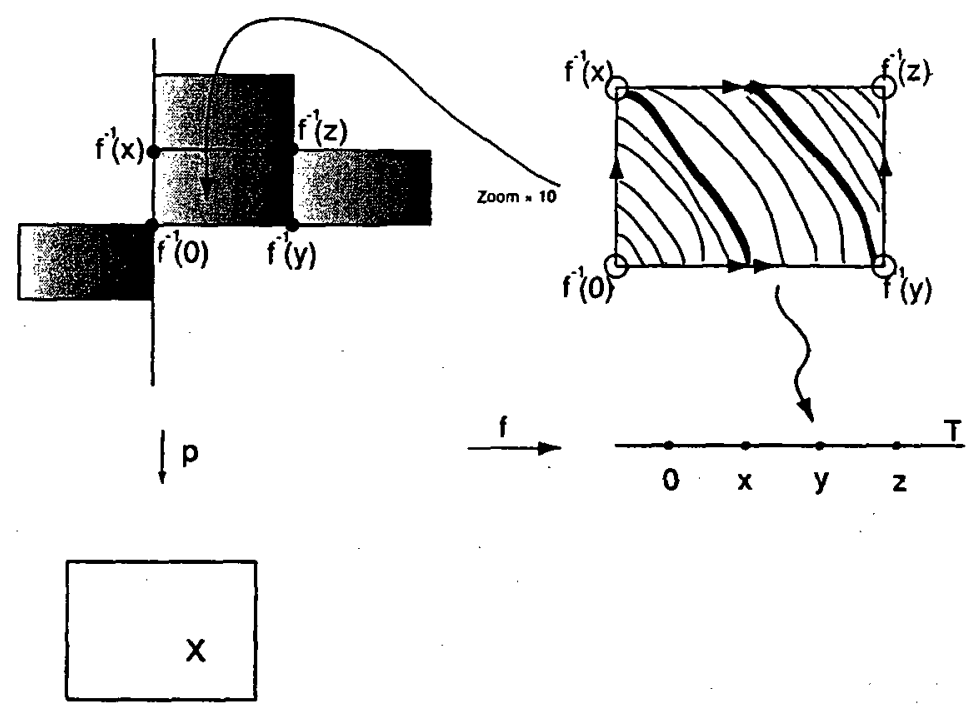

Figure 16: A band complex $X$ resolves the action $G$ on $T$

Pick a band $B=b \times I$ in a union of bands $Y$ underlying band complex $X$. Let $b \subset \alpha$ and $b^{*} \subset \beta$, where $\alpha, \beta$ are edges of underlying real graph $\Gamma$. Then $B$ determines an isometry $h_{B}: \alpha \rightarrow \beta$ whose restriction to $b$ is equal to $\delta_{B}: b \rightarrow b^{*}$. If we interchange the top and bottom of the band $B$, we have the inverse isometry $h_{B}^{-1}: \beta \rightarrow \alpha$. We say that $h_{B}$ is holonomy of $B$ if there is a vertical path $p$ in $B$ connecting $b$ to $b^{*}$. We say that $B$ is orientation-preserving (resp. orientationreversing) if its holonomy is orientation-preserving (resp. orientation-reversing).

Let a path $p: I \rightarrow L$, where $L$ is a leaf in $Y, x=p(0) \in b, y=p(1) \in c$, where $b, c$ are bases in $Y$, and let $N(x, \varepsilon)$ in $b$ push into $N\left(y, \varepsilon^{\prime}\right)$ in $c$. Then write a composition $p=p_{1} * \cdots * p_{n}$ of paths each of which is contained in a single band $B_{j}$. Thus, we can define the holonomy $h_{p}: b \rightarrow c$ of $p$ as the composition of holonomies of the paths $p_{j} . \quad p$ is orientation-preserving (resp. orientationreversing) if $h_{p}$ preserves (resp. reverses) the orientation.

If $b, c$ are contained in a single edge $\alpha=\beta$ of $\Gamma$ and $p$ is orientation-preserving, we define the translation length $l(p)$ of $p$ as the translation length of the isometry $h_{p}: \alpha \rightarrow \alpha$.

Now we can connect band complexes and trees by notions above. Suppose $X$ resolves an action of $G=\pi_{1}(X)$ on a tree $T$. We assume that every edge $\alpha$ of $\Gamma$ embeds isometrically into $T$ and that the path $p$ is such that $b, c$ are contained in a single edge $\alpha$. Define $\hat{p}$ of the path $p$ as the composition $\lambda * p$, where $\lambda$ is a path in $\alpha$ connecting $y$ to $x$. Then an element $g$ of $G=\pi_{1}(X, x)$ induced by $\hat{p}$ depends only on $p$.

REMARK 3.3 If the translation length of $p$ is not zero, then $g$ is a nontrivial element of $G$. In particular, let $U(L)$ be a simplicial component of $Y$ over some leaf $L$; if $p$ is a loop in $L$ based at $x$ in edge $\alpha$ then the element $g$ in $\pi_{1}(U(L), x)$ corresponding to $\hat{p}=p$ fixes $f(\tilde{x})$ in $T$; otherwise, if $g \cdot f(\tilde{x})=b \in T$ with $b \neq f(\tilde{x})$ then the pre-image of $b$ is a leaf not in $U(L)$, a contradiction. Futhermore, if $p$ is 
a vertical path in an annulus, then the element in $\pi_{1}(X)$ corrsponding to $p$ fixes an arc in $T$.

\subsubsection{A total space decomposition}

Definition 3.13 A stable $G$-tree $T$ is called pure if it admits a pure resolving band complex $X$, i.e., $X$ is minimal, the underlying union of bands $Y$ is connected, and $\pi_{1}(Y) \rightarrow \pi_{1}(X)$ is surjective.

THEOREM 3.2 Let $G$ be finitely presented, let $T$ be a stable $G$-tree, and let the action be stable. Suppose that a band complex $X$ is a resolution of an action of $G$. Let $Y$ be the underlying union of bands of $X$. Assume that $X$ has no faults. Then $G$ has a graph of groups decomposition such that:

1. the action on $T$ of each vertex group is either trivial or pure;

2. the action on $T$ of each edge group is trivial.

Proof. It is clear that the closure of $X \backslash Y$ has a natural CW-complex structure. We give $X$ the structure of a total space as follows :

1. the vertex spaces are the components of $Y$ and of the closure of $X \backslash Y$;

2. the edge spaces are the components of intersection of $Y$ and the closure of $X \backslash Y$. Note that each edge space is contained in a leaf of $Y$.

By Proposition 3.3, each component of $Y$ is either minimal or simplicial. If the component is minimal, then Remark 3.2 yields that the action of its fundamental group on $T$ is pure. If the component is simplicial, then it is an interval bundle over a leaf by Proposition 3.3 again. Thus, any loop in that component is homotopic to a loop in that leaf, and so the fundamental group of the component fixes a corresponding point in $T$ by Remark 3.3 ; in particular, the action of its fundamental group on $T$ is trivial.

Since each edge space is contained in a leaf of $Y$, the action of its fundamental group on $T$ is trivial by Remark 3.3 .

\subsubsection{The Kazhdan-Margulis lemma for resolutions of $\mathbb{R}$-trees}

DeFINITION 3.14 Let $J$ be a nondegenerate subarc of an edge of $\Gamma$ with a base point $z_{0}$ in the interior of J.A loop $\alpha$ in $X$ based at $z_{0}$ is $J$-short if it is $a$ composition $p_{1} * \lambda * p_{2}$, where $p_{1}, p_{2}$ are paths in $J$ and $\lambda$ is a path in a nonsingular leaf.

In this section, we assume that $X$ is a band complex with no faults. In the following two propositions, we assume that $C(Y)$ is a minimal component of $Y$ and write $C(\Gamma)=\Gamma \cap C(Y)$. 
Proposition 3.4 For each nondegenerate horizontal segment $J$ of $C(Y)$ and each $z$ in $C(Y)$, there is $\varepsilon$ such that $N(z, \varepsilon)$ pushes into $J$.

Proof. This immediately follows from the definition of minimality of $C(Y)$; every pushing-saturated subset is dense in $C(Y)$.

Proposition 3.5 Let $J$ be a nondegenerate subarc of an edge of $\Gamma$ with a base point $z_{0}$ in the interior of $J$. Then the image of $\pi_{1}(C(Y))$ in $\pi_{1}(X)$ is generated by $J$-short loops.

Proof. Recall that $Y$ has only finitely many singular leaves. Let $\gamma$ be a loop in $C(Y)$ based at $z_{0}$. Then $\gamma$ is homotopic to a composition of paths

$$
p_{0} * \lambda_{1} * p_{1} * \lambda_{2} * \cdots * \lambda_{k} * p_{k},
$$

where each $p_{i}$ is horizontal and each $\lambda_{i}$ is contained in a non-singular leaf. By the previous Proposition, each $p_{i}$ can be subdivided into segments $p_{i, j}$, each of which pushes onto $J_{i, j}$ in $J$. Futher, it can be ensured that the end points of $p_{i, j}$ lie in non-singular leaves because of the choice of finitely many singular leaves in $C(Y)$. Then $p_{i, j}$ is homotopic to a path of the form $\sigma_{i, j}^{+} * J_{i, j} * \sigma_{i, j}^{-}$, where each $\sigma_{i, j}^{ \pm}$is contained in a non-singular leaf. This gives the decomposition of $\gamma$ as a product of $J$-short loops.

Recall that if $T$ is a $H$-tree, then $T_{H}$ denotes the unique minimal $H$-invariant subtree in $T$.

THEOREM 3.3 Suppose that $X$ is a band complex resloving a stable G-tree $T$. Let $C(Y)$ be a minimal component of the underlying union of bands $Y$, and let $H$ denote the image of $\pi_{1}(C(Y))$ in $G=\pi_{1}(X)$. Then $T_{H}$ is stable. It follows that if $h \in H$ fixes an arc of $T$ then $h$ lies in the kernel of the action of $H$ on $T_{H}$.

Proof. Note that $H$ is finitely generated. Proposition 3.1 implies the existence of $T_{H}$. Let $J$ be an arc of $C(\Gamma)$ such that $J$ maps via the resolution $f: \tilde{X} \rightarrow T$ to an arc $f(J)$ in a maximal stable subtree $S$ in $T_{H}$. Let $\Delta_{J}$ be the set of generators of $H$ consisting of $J$-short loops constructed in the previous Proposition. For each generator $g \in \Delta_{J}, g(J)$ is horizontal; we can divide $g(J)$ into subarcs each of which pushes into $J$ and is homotopic to a path intersecting $J$ nondegenerately. Hence, $g(J) \cap J$ is nondegenerate arc as $J$ is nondegenerate; and so is $g(f(J)) \cap f(J)$ by $G$-equivariance. Then $g(S) \cap S$ contains a nondegenerate arc. Note that $g(S)$ is a stable subtree of $T_{H}$ as $S$ is a stable subtree of $T_{H}$. Proposition 3.2 implies that $g(S) \cup S$ is stable. But it contradicts to the maximality of $S$. Hence, $g(S)=S$, i.e., $S$ is $H$-invariant. However, $T_{H}$ is the unique minimal $H$-invariant subtree in $T$ under the action of $H$; hence $S=T_{H}$. Stability implies that if $h \in H$ fixes an arc in $S$, it fixes the whole $S$.

COROLLARY 3.1 Under the assumptions of theorem above, suppose that $\alpha, \beta \in H$ acting as hyperbolic isometries of $T$. If the length of $L=\operatorname{Axis}(\alpha) \cap \operatorname{Axis}(\beta)$ is greater than the sum of the translation lengths of $\alpha$ and $\beta$, then Axis $(\alpha)=$ $\operatorname{Axis}(\beta)$. 
ProOF. Consider the commutator $\gamma=\{\alpha, \beta]$. Let $J$ be a segment in $L$ adjacent to one of the end points with length $(J)=$ length $(L)-l(\alpha)-l(\beta)>0$. Then $\gamma$ fixes $J$. By the Theorem, $\gamma$ lies in the kernel of the action of $H$ on $T_{H}$.

Now suppose that $\operatorname{Axis}(\alpha) \neq \operatorname{Axis}(\beta)$. Then there is a nondegenerate segment $J^{\prime} \in \operatorname{Axis}(\beta) \backslash \operatorname{Axis}(\alpha)$ which is closed to $\operatorname{Axis}(\alpha) \cap \operatorname{Axis}(\beta)$. See Figure 17.

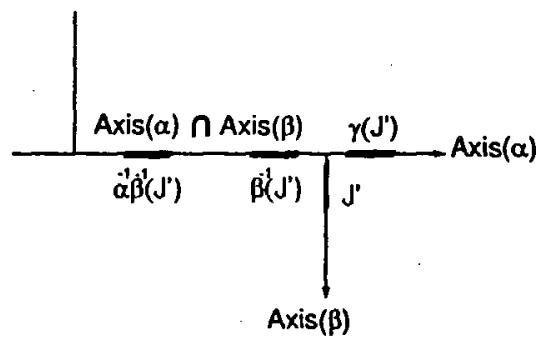

Figure 17: $\gamma\left(J^{\prime}\right) \in \operatorname{Axis}(\alpha) \backslash A x i s(\beta)$.

Hence, $\gamma$ does not fix $J^{\prime}$, which contradicts to $\gamma$ lies in the kernel of the action $H$ on $T_{H}$. The result follows.

\subsection{The machine}

\subsubsection{Moves}

Given a band complex $X$ resolving a $G$-tree, there are 6 moves that transform from $X$ into another one $X^{\prime}$ resolving the same action and $\pi_{1}(X) \cong \pi_{1}\left(X^{\prime}\right)$. Let $Y$ and $Y^{\prime}$ be the underlying union of bands of $X$ and $X^{\prime}$, respectively, and let $\Gamma$ and $\Gamma^{\prime}$ be the underlying real graph of $Y$ and $Y^{\prime}$, respectively.

Move M0 : Add a 2-cell. Attach a 2-cell to $X$ along a loop $\lambda$ in $Y \cup 1$-skeleton of $X$ such that $\lambda$ intersects $Y$ in a union of finitely many vertical fibers and $\lambda$ is null-homotopic in $X$. See Figure 18.

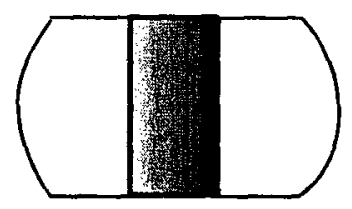

Figure 18: M0, the loop $\lambda$ is in bold and the added 2-cell is shaded.

Move M1 : Add an annulus. Let $J$ be a subarc of an edge of $\Gamma$. Attach an annulus $B$ to $J$; then attach a 2-cell along one of the vertical fibers of $B$. See Figure 19.

Move M2 : Subdivide a band. Let $B=[p, q] \times I$ be a band in $Y$, let $x$ be a point that lies in $(p, q)$. Then split $B$ down the fiber $x \times I$. We obtain $Y^{\prime}$ by replacing $B$ with the two bands $B_{1}=[p, x] \times I$ and $B_{2}=[x, q] \times I$. There are now two fibers over $x$, namely, $\sigma$ and $\sigma^{\prime}$. Attach a 2-cell along the loop $\sigma \cup \sigma^{\prime}$. See Figure 20. 


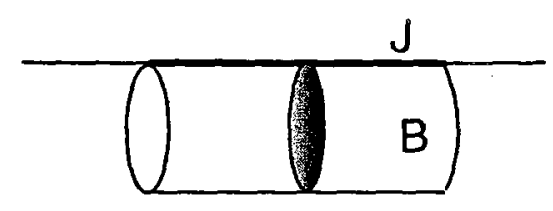

Figure 19: M1, the subarc $J$ is in bold and the added 2-cell is shaded.

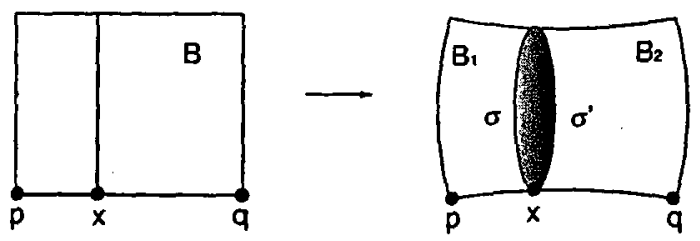

Figure 20: M2, subdividing a band along the fiber. The added 2-cell is shaded.

Move M3 : Split a point. Let $z$ be a point of $\Gamma$ which does not lie in interior of any bases. However, $z$ is a cut point and belongs to at least one base. Split $\Gamma$ at $z$, giving the graph $\Gamma^{\prime}$. The resulting graph $\Gamma^{\prime}$ has a collection of vertices $Z=\left\{z_{1}, z_{2}, \cdots, z_{s}\right\}$ corresponding to $z$. Attach the cone Cone $(Z)$ over $Z$ to $\Gamma^{\prime}$, as a collection of 1 -cells. Attach bands and cells to $\Gamma^{\prime} \cup$ Cone $(Z)$ as before, to get a band complex $X^{\prime}$. See Figure 21 .

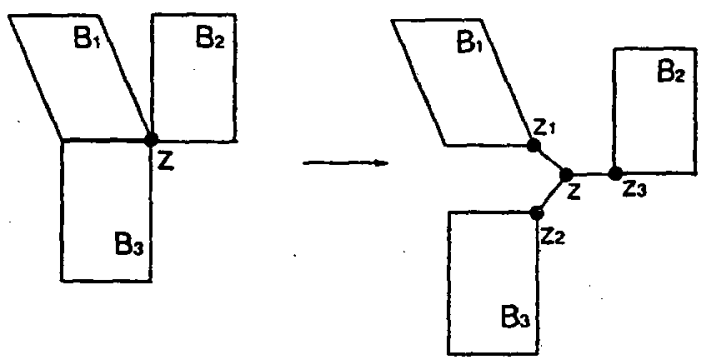

Figure 21: M3, splitting a point. Cone $(Z)$ is the collection of 1-cells $\left\{z z_{1}, z z_{2}, z z_{3}\right\}$.

LEMMA $3.2 B y$ applying a finite squence of moves M2 and M3 to a band complex $X$, it ensures that $X$ contains no faults.

Proof. We recall that according to out discussion in Proposition 3.3, $X$ has only finitely many faults each of which contains a critical point.

First, we are using M2 to eliminate all dead ends. Note that this may creat cut points (for example in the situation of Figure 12, in $L_{2}$ ). Then all cut points can be removed by M3. This will not creat dead ends. Therefore, we get a band complex without faults.

Move M4 : Slide. Let $B=b \times I$ and $C=c \times I$ be distinct bands in $X$. Suppose that $f_{c}(c) \subset f_{b}(b)$. We change $Y$ to $Y^{\prime}$ by changing $f_{c}$ to $f_{b^{*}} \circ \delta_{B} \circ f_{b}^{-1} \circ f_{c}$. Informally, we slide the base $c$ from $b$ to $b^{*}$ along the band $B$. As a result, the band $C$ in $X$ transforms to a band $C^{\prime}$ in $X^{\prime}$. Note that there is a homotopy between $X$ and $X^{\prime}$, so $\pi_{1}(X) \cong \pi_{1}\left(X^{\prime}\right)$. We say that $b$ is the carrier of the slide and $c$ is the carried base. See Figure 22. 


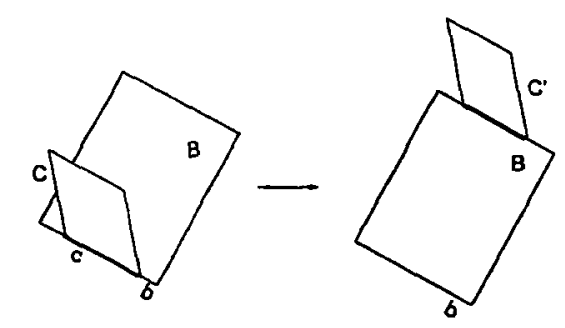

Figure 22: M4, a slide.

REMARK 3.4 To avoid the increase of complexity, we should slide carried base $c$ that has weight 0 or $\frac{1}{2}$ along $B$ both bases of the band $C$ in a single move.

LEMMA 3.3 Suppose that we have a nondegenerate arc $J$ of the underlying real graph $\Gamma$ in the band complex $X$ such that $J$ pushes onto an arc $J^{\prime}$ in $\Gamma$. Then there is a finite sequence of moves $M 1$ and $M 4$ that transforms $X$ into a band complex $X^{\prime}$ with underlying union of bands $Y^{\prime}$ equals $Y \cup B$, where $Y$ is the underlying union of bands of $X$ and $B$ is a band with bases $J, J^{\prime}$.

Proof. First, we attach an annulus to $J$ using M1. Now keep one of bases of annulus attached to $J$. Then the push of $J$ onto $J^{\prime}$ is actually a sequence of moves M4 when we slide another base of annulus onto $J^{\prime}$.

The following lemma is used to descried the next Move M5 and it provides a way to simplify Möbius bands.

Lemma 3.4 Let $B=b \times I$ be a Möbius band. Let $x \in f_{b}(b)$.

1. If $x$ is the midpoint of the base, then there is a sequence of moves that $B$ can be split over $x$ to form a band of weight 1 and an annulus.

2. If $x$ lies in the interior of a half-base, then there is a sequence of moves that $B$ can be split over $x$ to form an annulus, $a$ Möbius band and $a$ band of weight 1 .

Proof. In the first case, subdivide $B$ along the fiber $x \times I$ into two weight 1 bands by using M2. Then slide one of these bands over the other one by using M4, creating an annulus. See Figure 23. The second case is similar to the first one.

\section{Definition $3.15 A$ subarc $J$ of $a$ base $b$ is free if either}

1. $b$ has weight 1 and the interior of $J$ does not intersect any other base of positive weight, or

2. $b$ has weight $\frac{1}{2}$, the interior of $J$ does not contain the mindpoint of $b$, and the bases $b, b^{*}$ are the only positive weight bases that intersect the interior of $J$. 


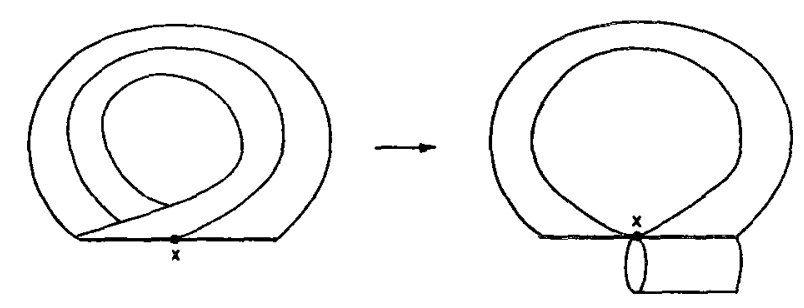

Figure 23: Simplifying a Möbius band in case 1

Move M5 : Collapsing from a free subarc. Let $B=b \times I$ be a band in $Y$ and $J$ be a free subarc of $b$.

1. First, use M2 to subdivide all annuli intersect $J$, until a base of new creating annuli is contained in $J$. Let $X_{1}$ be the resulting band complex.

2. Next, use M4 to slide all new annuluses over $B$ to $\delta_{B}(J)$. Let $X_{2}$ denote the resulting band complex.

3. If weight of $b$ is 1 , then collapse $J \times I$ to $\delta_{B}(J) \cup \partial J \times I$, where $\partial J$ denotes the boundary of $J$. In the special case where an end point $x$ of $J$ is also an end point of $b$ and the open vertical fiber $x \times(0,1)$ does not intersect any cells in $X_{2}$, we eliminate $x \times(0,1)$. If weight of $b$ is $\frac{1}{2}$, use Lemma 3.4 to simplify the band $B$ over the end point $x$ of $J$ nearest the midpoint of $b$. Now $J$ is contained in a weight 1 base, and we can collapse as before. This result is the band complex $X^{\prime}$

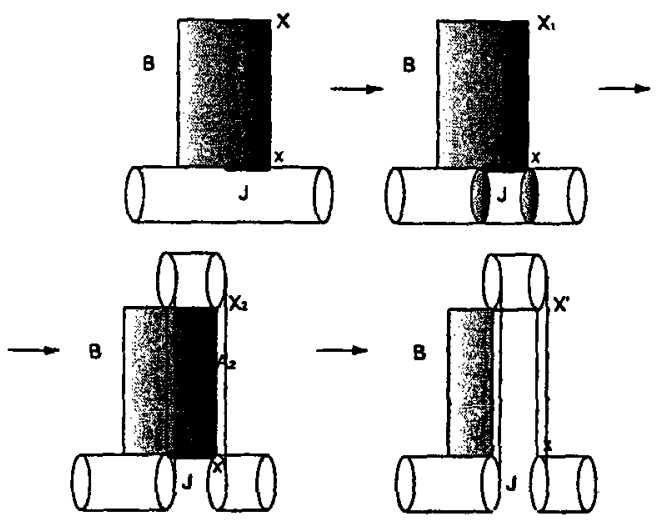

Figure 24: M5, collapsing a weight 1 band from a free subarc $J$

Note that $A_{1}$ and $A_{2}$ are 2-cells. We call $A_{1}, A_{2}$ subdivision annuli in $X_{2}$.

\subsubsection{Preliminary assumptions and outline}

We start with a finitely presented group $G$. Let $X$ be a band complex with underlying union of bands $Y$ and real graph $\Gamma$. Suppose that $X$ resolves an stable action $G$ on an $\mathbb{R}$-tree $T$. 
We now use moves M2 and M3 to transform $X$ into another band complex (which we still denote $X$ ) such that the new band complex $X$ satisfies the following properties :

A1 The real graph $\Gamma$ is the disjoint union of its edges. Each edge is a block. This can be achieved via move M3.

A2 $X$ has no faults, by Lemma 3.2.

A3 $Y$ is a disjoint union of components each of which is either minimal or simplicial, each simplicial component is an interval bundle, followed by Lemma 3.2 and Proposition 3.3.

A4 If $A=a \times I$ and $B=b \times I$ are Möbius bands such that the length of $a$ is greater than the length of $b$ and $a, b$ share midpoints, then $B$ is equal to the remaining Möbius band in $A$ by sliding arcs (from the left (resp. right) end point $a$ to the left (resp. right) end point $b$ ) using Lemma 3.4. Clearly, if the length of $a$ is equal to the length of $b$, then $B=A$.

A5 The resolution $f: \bar{X} \rightarrow T$ embeds lifts of bases into $T$, where $\tilde{X}$ is the universal cover of $X$.

\subsubsection{Process I}

To describe the process, we have to start with the following definitions.

Definition $3.16 \quad$ 1. A weight 1 base $b$ is isolated if it is a free subarc of itself. Half of a weight $\frac{1}{2}$ base $b$ is isolated if it is a free subarc of $b$.
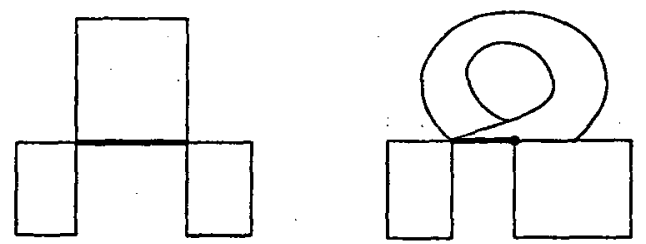

Figure 25: An isolated base and an isolated half-base, marked by the bold lines.

2. $A$ weight 1 base $b$ is semi-isolated if one of its endpoints is contained in a free subarc of $b$. Half of a weight $\frac{1}{2}$ base $b$ is semi-isolated if one of the endpoints of the half-base is contained in a free subarc.
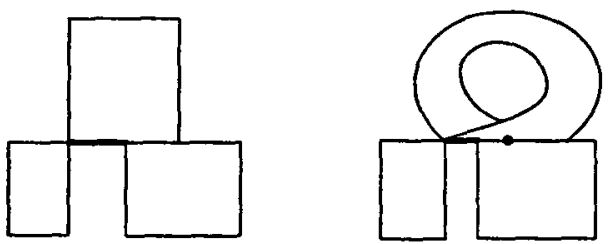

Figure 26: A semi-isolated base and a semi-isolated half-base is in bold lines. 
Definition $3.17 A$ long band $B$ is a sequence of weight 1 bands $B_{1}, \cdots, B_{n}$ with the following are satisfied:

1. the top of $B_{j}$ is contained in the bottom of $B_{j+1}$ for $j=1,2, \cdots, n-1$;

2. $B_{i} \cap B_{j}=\varnothing$ if $|i-j|>1$;

3. $B_{1} \cup \cdots \cup B_{n}$ intersects other bands of positive weight in a subset of the union of the bottom of $B_{1}$ and the top of $B_{n}$.

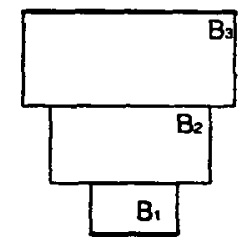

Figure 27: A long band $B=B_{1} \cup B_{2} \cup B_{3}$, where the top and bottom of $B$ is in bold.

We can now start the machine. The Process I takes a band complex $X$ and creates a new one $X^{\prime}$. Process $I$ is applied to a minimal component $C(Y)$ of underlying union of bands $Y$.

Process I Find a maximal free subarc $J$ of a base of $C(Y)$. Then use move M5 to collapse from $J$. If there does not exsit a free subarc of a base of $C(Y)$, then go to Process II. Choose $J$ according to the following rules :

R1 If there is an isolated (half-)base, choose that for $J$ (there might be several choices for $J$, choose one at random).

R2 If there does not exist any isolated (half-)bases, but there is a semi-isolated (half-) base, choose that for $J$.

R3 If there does not exist any isolated or semi-isolated (half-)bases, choose any maximal free subarc for $J$.

REMARK 3.5 If we have a long band $B=B_{1} \cup \cdots \cup B_{n}$ and $J$ is the bottom of $B_{1}$, then collapsing from $J$ by move $M 5$ is $n$ consecutive collapses, i.e., collapse from $J$, then from the bottom of $B_{2}$, etc., and finally from the bottom of $B_{n}$. These $n$ operations are treated as a single move producing $X^{\prime}$.

LEMMA 3.5 Process I does not increase complexity.

Proof. Let $X^{\prime}$ be a band complex produced from a band complex $X$ by Process $\mathrm{I}$, and let $\sigma$ denote the complexity. We need to show that $\sigma\left(X^{\prime}\right) \leq \sigma(X)$.

We first consider the case when R1 applied. Let $B$ be the band with a bottom isolated (half-)base $b$, and let $\gamma$ be the block containing $b$. Then after collapse 


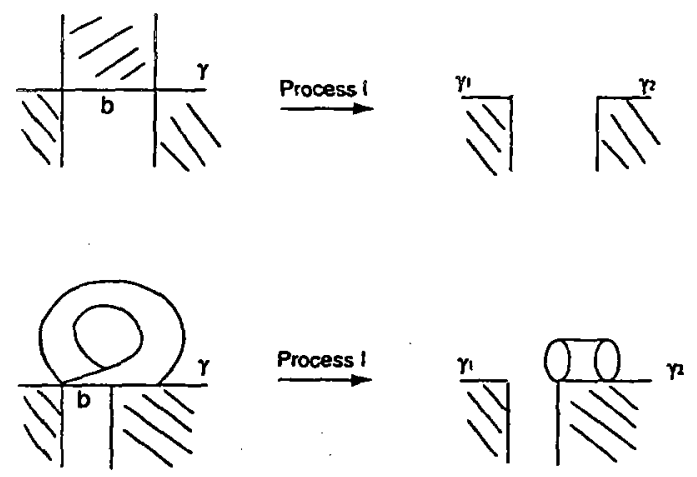

Figure 28: R1 of Process I does not increase complexity

from $b$, the block splits into two components $\gamma_{1}, \gamma_{2}$ of positive complexity. See the following figure. Then after collapsing from $b$, we have $\sigma(\gamma)>\sigma\left(\gamma_{1}\right)+\sigma\left(\gamma_{2}\right)$. Note that if $B$ is a Möbius band, we first use Lemma 3.4 to get a band of weight 1 and an annulus then collapse the weight 1 base by Process I.

Similarly, for the case when R2 applied, the result follows.

Now let's consider when R3 applied. Let $B$ be the band with a bottom base $b$ containing the maximal free subarc $J$, and let $\gamma$ be the block containing $b$. Then after collapse from $J$, the block splits into two or three components depending on the location of $\delta_{B}(J)$. Without loss the generality, we assume that $\gamma$ splits into two components $\gamma_{1}, \gamma_{2}$ of positive complexity and $\delta_{B}(J)$ is contained in a block $\alpha \neq \gamma$ with positive complexity. Let $\alpha^{\prime}$ be obtained from $\alpha$ after collapsing from $J$. Note that $\alpha^{\prime}$ is still a single block. See the below figure. Then after collapsing
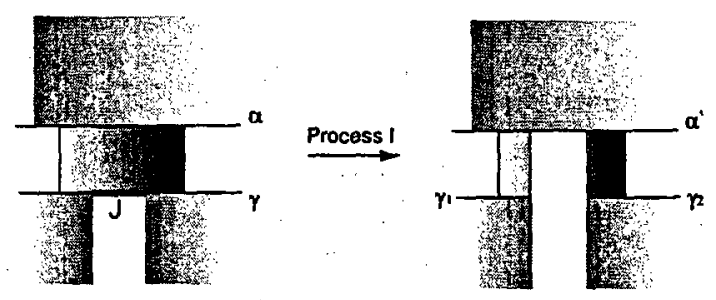

Figure 29: R3 of Process I does not increase complexity

from $J$, we have

$$
\sigma\left(\gamma_{1}\right)+\sigma\left(\gamma_{2}\right)=-3+\sum_{c \subset \gamma} w(c)=\sigma(\gamma)-1
$$

where $c$ is a base in $\gamma$, and

$$
\sigma\left(\alpha^{\prime}\right) \leq \sigma(\alpha)+1
$$

Therefore,

$$
\sigma\left(\gamma_{1}\right)+\sigma\left(\gamma_{2}\right)+\sigma\left(\alpha^{\prime}\right) \leq \sigma(\gamma)+\sigma(\alpha)
$$

The following proposition gives the result of the application of Process $\mathrm{I}$. 
PROPOSITION 3.6 Let $X_{0}, X_{1}, \cdots$ be an infinite sequence of band complexes such that $X_{i+1}$ is obtained from $X_{i}$ by applying Process $I$ for each $i>0$. Then the following holds :

1. The number of weight $\frac{1}{2}$ bases does not increase.

2. The choice of $J$ in $R 1$ occurs only finitely many times.

3. There is a number $N$ such that all bands in each $X_{i}$ can be organized into at most $N$ long bands.

4. For any $n$, there exists a segment of length $n$ contained in some leaf such that this segment is eventually collapsed.

5. The choice of $J$ in $R 3$ occurs infinitely many times.

PROOF.

1. This follows from Lemma 3.4 .

2. Collapse from an isolated (half-)base $J$ in a band $B$ will eliminate one block or decrease the complexity of the block containing $\delta_{B}(J)$. (See Figure 28). Hence, the complexity of band complex will decrease, and so $J$ can only be chosen finitely many times.

3. Note that the choice of R1 or R2 implies the number of long bands is bounded in the sequence of $X_{0}, X_{1}, \cdots$ after finitely many steps.

Now consider only the choice of R3 applied. Let $\gamma$ be a block containing a free subarc in $X_{i}$. Then the number of long bands in $\gamma$ is the complexity of $\gamma$ plus two. Note that the complexity of $\gamma$ is greater than zero. Hence, the number of long bands in $\gamma$ is less or equal to three times the complexity of $\gamma$. The result follows.

4. Define $N(k)$ inductively for $k \in \mathbb{N}$. Set $N(0)=0$. Let $N(k+1)$ be the least integer such that all points on free subarcs of $\Gamma \cap X_{N(k)}$ are eventually collapsed at stage $N(k+1)$. Note that this is well-defined because there are only finitely many maximal free subarcs at any stage. Thus, each point in $X_{0}$ that is collapsed at stage $N(n)$ belongs to a vertical segment of length at least $n$ in $X_{0}$ which has been collapsed after stage $N(n)$ of Process I.

5. Suppose that only R2 applied. Then the number of positive weight bands in Process I remains constant $\eta$. We need to show that the assumption implies that Process I will terminate after finitely many steps. So it contradicts to an infinite sequence of band complexes.

From (4), there exist a vertical segment $\lambda$ of length $n>4 \eta$ which is eventually collapsed at stage $N(n)$. Then $\lambda$ must intersect a band $B=b \times I$ in at least five distinct vertical segments. Among these five segments, we choose three segments $\lambda_{1}, \lambda_{2}, \lambda_{3}$ that have the following properties : 
According to the orientation of $\lambda, \lambda_{1}$ is the first and $\lambda_{3}$ is the last. Let $x_{i}$ be the initial point of $\lambda_{i}$ in $b$. Then the paths $\left[x_{1}, x_{2}\right]$ and $\left[x_{2}, x_{3}\right]$ must have the same orientation.

Let $\theta>0$ be the minimum horizontal distance that separates segments $\lambda_{1}, \lambda_{2}, \lambda_{3}$. Then $\lambda_{2}$ lies at least $\theta$ from $\lambda_{1}$ or $\lambda_{3}$. At stage $N(n), \lambda$ is collapsed and only R2 applied. Thus, after stage $N(n)$, the length of $b$ has decreased by at least $\theta$. It follows inductively that between stages $N(k n)$ and stages $N((k+1) n)$, at least one base is reduced in length by at least $\theta$. This implies that eventually all bases will vanish and Process I will terminate. A contradiction.

\subsubsection{Process II}

Now we describe Process II. Let $C(Y)$ be a minimal component of $Y$, and write $C(\Gamma)=\Gamma \cap C(Y)$. Suppose that Process I does not apply to $C(Y)$. Then

(*) the weight of each point $z \in C(\Gamma)$ is at least 2 , i.e., $w(z) \geq 2$.

Otherwise, $z$ would be contained in a free subarc.

Process II We identify the components of $C(\Gamma)$ with disjoint closed intervals in $\mathbb{R}$. This gives $C(\Gamma)$ a total order. Let $F$ be the first component of $C(\Gamma)$ and $x$ be the initial point of $F$. Let $b$ be the longest positive weight base containing $x$. If possible, take $b$ to have weight 1 (if there are several such bases, then choose one at random). Now produce the new band complex $X^{\prime}$ as follows :

Operation 1 Slide over $b$ all those positive weight bases $c$ whose midpoint is moved away from $x$ after the slide. The base $b$ is the carrier of each of these slides and the base $c$ is carried during this operation.

Operation 2 Collapse from the maximal free initial segment $J$ of $b$. Note that the segment $J$ is now necessarily nondegenerate.

If the complexity decreases, we stop Process II and go back to Process I.

The bands $b \times I$ and $c \times I$ are said to have participated in Process II. We retain the name of bases $b$ and $c$ in $X^{\prime}$ obtained from $b$ by collapsing the segment $J$ and sliding along $B$, respectively.

Proposition 3.7 Suppose $X^{\prime}$ results from $X$ via Process $I I$.

1. The complexity of $X^{\prime}$ is no greater than the complexity of $X$.

2. If the complexity of $X^{\prime}$ equals the complexity of $X$, then (*) holds for $X^{\prime}$. 
PROOF.

1. The collapse in Operation 2 cannot increase the complexity by Lemma 3.5 in Process I. But slides in Operation 1 may increase the complexity. See Figure below. To avoid this case, we use Operation 2 to eliminate the carrier base

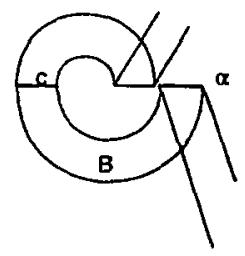

Figure 30: After sliding $c$ along $B, \sigma\left(X^{\prime}\right)$ is increased.

after all those slides in Operation 1. Thus, collapsing reduces complexity again.

2. Prove by a contradiction. Suppose that $(*)$ does not hold for $X^{\prime}$. This can occur if after sliding a base $c$ along $B$ in Operation 1 becomes a base of weight 0 or $\frac{1}{2}$. However, in this case, complexity is reduced, contradicting the complexity of $X$ is equal to the complexity of $X^{\prime}$. See Figure 31. Let

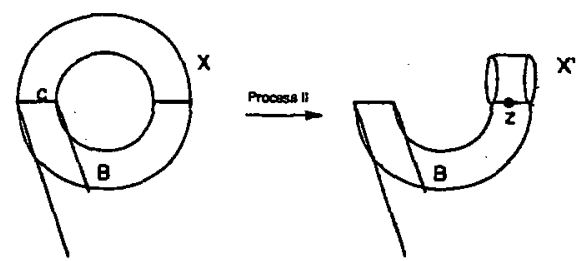

Figure 31: $w(z)<2$ implies that $\sigma(X)<\sigma\left(X^{\prime}\right)$.

$J$ be the maximal free subarc of a base $b$ that is collapsed by Operation 2 . We could have the situation : after all slides, the weight of $z \in \delta_{B}(J)$ less than three, so collapsing in Operation 2 would reduce the weight of $z$ by one. However, this would imply that the base $b$ of $X$ contains a free subarc, in which case it must be done by Process $I$ in $X$. See Figure below.

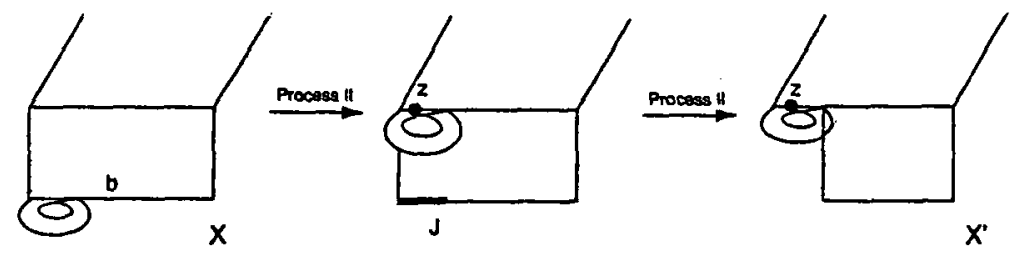

Figure 32: Another case for $w(z)<2$.

This guarantees that the machine works properly and one of process will be applied. Next, we will see some properties of Process II. 
Proposition 3.8 Let $X_{0}, X_{1}, \cdots$ be a sequence of band complexes such that for each $i \geq 0$, the complex $X_{i+1}$ is obtained from $X_{i}$ via Process II. Then one of following holds :

1. The sequence terminates after finitely many steps.

2. The surface case : For all sufficiently large $i$ and each point $z$ in $\Gamma_{i}=$ $\Gamma\left(X_{i}\right)$ which is not a end point of a base, we have $w(z)=2$.

3. The toral case :

(a) Every base has a limiting position in $\Gamma$.

(b) Some base $b$ is a carrier and is carried infinitely many times and has length that does not converge to zero.

(c) The bases $b$ and $b^{*}$ have the same limiting positions.

Proof. Suppose that neither 1 nor 2 occur. Then we need to show that the toral case occurs. Since Proposition $3.7 \sigma\left(X_{i+1}\right) \leq \sigma\left(X_{i}\right)$ for all $i$ and Process II will go back to Process I if $\sigma\left(X_{i+1}\right)<\sigma\left(X_{i}\right)$, we have the complexity of this sequence of band complexes is constant.

(a) Recall that we identify $\Gamma$ with a disjoint union of intervals in $\mathbb{R}$. Since on each step of Process II we have that every base either is carried, and hence it moves to the positive direction in $\mathbb{R}$ and its length remains constant, or is the carrier, and thus the right end point of that base does not move and its length does not increase. This proves (a).

Without loss of generality, we may assume the following :

(i) If a band participates in Process II, it does so infinitely many times. (Otherwise, Process II will terminate.)

(ii) All weights of bases remain constanst as a result of Process II. (Otherwise, the complexity will decrease and Process II will terminate.)

(b) Let $D_{i}$ denote the total measure of the subset of points of $\Gamma_{i}$ between the initial point and the minimal point of $\Gamma$ which lies in a band and never participates. Also let

$$
\tau_{i}=\sum_{b}\left\{l(b) w(b) ; b \text { is a base of } X_{i} \text { that eventually participates }\right\}
$$

where $l(b)$ is the length of $b$ and $w(b)$ is the weight of $b$. Now set

$$
\operatorname{excess}\left(X_{i}\right)=\tau_{i}-2 D_{i}
$$


We claim that $\operatorname{excess}\left(X_{i}\right)>0$ and $\operatorname{excess}\left(X_{i}\right)=\operatorname{excess}\left(X_{i+1}\right)$.

Indeed, the first assertion follows from $(*)$ and the surface case does not apply. For the second assertion, we analyzc Operation 1 and Operation 2. As a result of Operation 1, we slide bands over each other, and this does not change their weights by assumption (ii). On the other hand, during Operation 2, where we collapse the maximal free subarc $J$ contained in the carrier $d$, we have excess $\left(X_{i+1}\right)=\left(\tau_{i}-2 l(J) w(d)\right)-\left(2 D_{i}-2 l(J) w(d)\right)=$ $\operatorname{excess}\left(X_{i}\right)$. This proves the claim.

The claim implies that there is a base $b$ participates infinitely many times and its length does not converge to zero. Without loss of generality, we can assume that each time the base $b$ participates, it is the carrier. Thus we need to show that $b$ is carried infinitely many times. Suppose not. Then $b^{*}$ is carried finitely many times. Consider that $b$ and $b^{*}$ are carried zero times. Then if $b$ and $b^{*}$ have disjoint interiors, any base can only be carried by $b$ finitely many times, contradicting Process II never terminate. Likewise, if $B=b \times l$ is orientation-reversing, any base can only be carried by $b$ finitely many times, also a contradiction. Therefore suppose $B$ has weight 1 and is orientation-preserving. Then the translation length $\nu>0$ of $B$, and some base gets translated by $\nu$ infinitely many times. This contradicts to (a).

(c) If the base $b$ has weight $\frac{1}{2}$, then we are done. Suppose weight of $b$ is 1 . Let $\nu_{i}$ be the distance between the midpoints of $b$ and $b^{*}$. Then any base is carried by $b$ in a distance $\nu_{i}$. Therefore, the distance $\nu_{i}$ must tend to zero as $i$ goes to infinite in Process II.

\subsection{Machine Output}

Let $T$ be an $\mathbb{R}$-tree, $G$ be a finitely presented group acting on $T$. We construct a band complex $X$ that resolves an action of $G=\pi_{1}(X)$ on tree $T$ as in Theorem 3.1. Let $Y$ be the underlying union bands of $X$ and $C(Y)$ be a minimal component of $Y$. Applying the machine to $C(Y)$, there are three possibilities.

\section{Definition 3.18 1. The thin case, in which eventually only Process $I$ is applied.}

2. The surface case, in which eventually only Process II is applied and excess $=0$.

3. The toral case, in which eventually only Process II is applied and excess > 0 .

Let $\bar{Y}$ denote the union of bands $Y$ with the annuli removed. Given a component $C(Y)$ of $Y$, write $\overline{C(Y)}$ for $\bar{Y} \cap C(Y)$. Let $i$ denote the homomorphism $i: \pi_{1}(C(Y)) \rightarrow G$ induced by the inclusion; and let $H=i\left(\pi_{1}(C(Y))\right)$. Let $N$ be the normal subgroup normally generated by the images of the fundamental groups of annuli in the closure of $C(Y) \backslash \overline{C(Y)}$, and let $\bar{H}=H / N$. 


\subsubsection{The surface case}

Recall that a short exact sequence of groups $A, B$, and $C$ is given by two maps $\alpha: A \rightarrow B$ and $\beta: B \rightarrow C$ and is written

$$
1 \rightarrow A \rightarrow B \rightarrow C \rightarrow 1
$$

Because it is an exact sequence, $\alpha$ is injective, and $\beta$ is surjective. Moreover, the group kernel of $\beta$ is the image of $\alpha$. Hence, the group $A$ can be considered as a (normal) subgroup of $B$, and $C$ is isomorphic to $B / A$.

Proposition 3.9 Suppose $C(Y)$ is in the surface case. Then the following holds:

1. For each leaf $L$ of $C(Y)$, the image of $\pi_{1}(L)$ in $G=\pi_{1}(X)$ admits a map onto a cyclic group such that the kernel is contained in the kernel of the action of $H$ on $T_{H}$.

2. Suppose that $X$ is pure. Then there is a short exact sequence

$$
1 \rightarrow \operatorname{Ker}(G \curvearrowright T) \rightarrow G \rightarrow \pi_{1}(O) \rightarrow 1
$$

where $G \curvearrowright T$ is the action $G$ on $T$ and $O$ is $\bar{Y}$ with some 2-cells attached.

Proof. Because $C(Y)$ is in the surface case, we have that only Process II is applied to $C(Y)$ and excess $(C(Y))=0(w(z)=2$ for each point $z \in C(\Gamma)$ that is not a end point of a base). We may assume that there are no Möbius bands by Lemma 3.4. Therefore, $\overline{C(Y)}$ looks like the Figure 33.

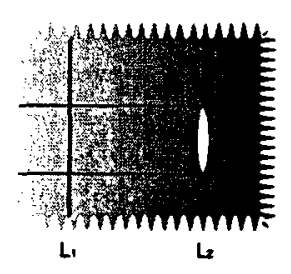

Figure 33: The surface case $\overline{C(Y)}$ with a non-singular leaf $L_{1}$ and a singular leaf $L_{2}$ in bold.

Let $L$ be a leaf in $\overline{C(Y)}$. If $L$ is non-singular leaf, then it is homeomorphic to $\mathbb{R}$; if $L$ is singular leaf, then it is a circle with finitely many rays attached because the definition of singular leaves and the our assumption $X$ contains no faults. See the Figure 33 again. Hence all leaves in $\overline{C(Y)}$ have cyclic fundamental group.

Let $L$ be a closed vertical loop in an annulus of $C(Y)$. Then the image of $\pi_{1}(L)$ in $G$ is a cyclic subgroup which fixes an arc in $T$ by Remark 3.3, and so by Theorem 3.3 (Kazhdan-Margulis Lemma) lies in the kernel of the action of $H$ on $T_{H}$.

Now, let $L$ be a general leaf in $C(Y)$. Then $\pi_{1}(L)$ has a normal subgroup $N \cap \pi_{1}(L)$ which is contained in the kernel of the action on $T_{H}$; and the quotient group that corresponds to $L \cap \overline{C(Y)}$ is cyclic. This proves the first assertion. 
Now consider the second assertion. Since $X$ is pure, we have that $X$ is minimal, $C(Y)=Y$, and $H=G$. There is a short exact sequence

$$
1 \rightarrow N \rightarrow G \rightarrow \bar{H} \rightarrow 1
$$

Then $N=\operatorname{Ker}(G \curvearrowright T)$ because clearly $N \subset \operatorname{Ker}\left(H \curvearrowright T_{H}\right)$ and annuli are the only way in which trivial actions can happen; and $\bar{H}$ is clearly the fundamental group of $\bar{Y}$ with some 2-cells attached.

\subsubsection{The toral case}

Let $C(Y)$ be a toral component of the union of bands $Y$ underlying the band complex $X$, and let $X=X_{1}, X_{2}, \cdots$ be a sequence of band complexes obtained by applying Process II to $C(Y)$. By Proposition 3.8, we may assume that for each $i$ the component $C\left(Y_{i}\right)$ has only one block. Let $J$ denote the block of $C(Y)$. Note that each base $b$ of $C\left(Y_{i}\right)$ determines a partial isometry $h(b)$ of $J$, which can be extended to an isometry of $\mathbb{R}$. Thus, $C\left(Y_{i}\right)$ determines a finitely generated group $\mathcal{B}_{i}$ of isometries of $\mathbb{R}$. Note that $\mathcal{B}_{i}=\mathcal{B}_{j}$ for all $i, j$ since slides correspond to compositions of elements and collapses of proper subarcs do not change the group. Denote this constant group $\mathcal{B}$ that is called Bass group of $C(Y)$. Clearly; each element of $\mathcal{B}$ is either a translation or a reflection.

LEMMA 3.6 Let $\mathcal{B}$ be a finitely generated group of isometries of $\mathbb{R}$.

1. Let $\tau_{j}: x \mapsto x+t_{j}$ be a sequence of translations in $\mathcal{B} \backslash\{1\}$ such that $\lim _{j \rightarrow \infty} t_{j}=0$. Then this sequence contains translation $\tau_{i}, \tau_{j}$ such that the translation lengths of $\tau_{i}$ and $\tau_{j}$ is irrational, i.e., $t_{j} / t_{j}$ is irrational.

2. Let $\sigma_{j}$ be a sequence of reflections in $\mathcal{B} \backslash\{1\}$ such that lim $_{j \rightarrow \infty} \sigma_{j}=\sigma_{0}$ where $\sigma_{0} \in \mathcal{B}$. Then there exists $\sigma_{i}, \sigma_{j}, \sigma_{k}$ such that the ratio of the translation lengths of $\sigma_{i} \sigma_{j}$ and $\sigma_{i} \sigma_{k}$ is irrational.

LEMMA 3.7 Moves MO-M4 can be applied to transform $X$ to a band complex $X^{\prime}$ such that there are two orientation-preserving bands $A=a \times I$ and $A^{\prime}=$ $a^{\prime} \times I$. They determine partial isometries $\alpha, \alpha^{\prime}$ of $J$ such that the domain of the composition $\alpha \alpha^{\prime} \alpha^{-1}\left(\alpha^{\prime}\right)^{-1}$ is a nondegenerate segment and the ratio of the translation lengths of $\alpha$ and $\alpha^{\prime}$ is irrational.

Proof. By Proposition 3.8, there is a base $b$ that is carrier and is carried infinitely many times in the sequence $X_{i}$. Let $b_{i}$ denote the base in $X_{i}$ corresponding to $b$. Then the sequence $b_{i}$ converge to a nondegenerate segment; and so the corresponding partial isometries $h\left(b_{i}\right) \in \mathcal{B}$ of $J$ form a converge sequence and the limit is the identity in the orientation-preserving case. In particular, eventually all $b_{i}$ are either orientation-preserving or orientation-reversing because of the limiting position of the sequence $b_{i}$, so is $h\left(b_{i}\right)$. 
Case I : Suppose that all $h\left(b_{i}\right)$ are orientation-preserving. By Lemma above, there are bases $b_{i}, b_{j}$ such that the ratio of the translation lengths of $h\left(b_{i}\right), h\left(b_{j}\right)$ is irrational. Use Lemma 3.3 to attach a band $A=a \times I$ and $A^{\prime}=a^{\prime} \times I$ to $J$ by identifying $a$ and $a^{*}$ with $b_{i}$ and $b_{i}^{*}$ respectively, and identifying $a^{\prime}$ and $a^{*}$ with $b_{j}$ and $b_{j}^{*}$ respectively. Now denote the partial isometries $\alpha, \alpha^{\prime}$ by the restriction of $h\left(b_{i}\right), h\left(b_{j}\right)$ to $b_{i}, b_{j}$. Recall that the limiting position of $b_{i}$ and $b_{i}^{*}$ is the same by Proposition 3.8. Thus we have

$$
\lim _{i \rightarrow \infty} b_{i}=\lim _{j \rightarrow \infty} b_{j}, \quad \lim _{i \rightarrow \infty} h\left(b_{i}\right)=\lim _{j \rightarrow \infty} h\left(b_{j}\right)=i d .
$$

It follows that for $i, j$ sufficiently large, the domain of the composition $\alpha \alpha^{\prime} \alpha^{-1}\left(\alpha^{\prime}\right)^{-1}$ is a nondegenerate segment.

Case II : Suppose that all $h\left(b_{i}\right)$ are orientation-reversing. Similarly, by using Lemma above, we find $b_{i}, b_{j}, b_{k}$ such that the ratio of translation lengths of $h\left(b_{i}\right) h\left(b_{j}\right)$ and $h\left(b_{i}\right) h\left(b_{k}\right)$ is irrational. Choose $i, j, k$ large enough such that $b_{i j}=$ $b_{i} \cap b_{j}$ and $b_{i k}=b_{i} \cap b_{k}$ are nondegenerate segments. Then $B_{i j}=b_{i j} \times I \subset b_{j} \times I=B_{j}$ and $B_{i k}=b_{i k} \times I \subset b_{k} \times I=B_{k}$. We use M2 to subdivide the bands $B_{j}, B_{k}$ so that the bands $B_{i j}, B_{i k}$ are bands in the new complex $X^{\prime}$. Now use M4 to slide $b_{i j}, b_{i k}$ from $b_{i}$ to get orientation-preserving bands $A, A^{\prime}$ such that their translation lengths are equal to the translation lengths of $h\left(b_{i}\right) h\left(b_{j}\right)$ and $h\left(b_{i}\right) h\left(b_{k}\right)$. For $i, j, k$ sufficiently large, the domain of $\alpha \alpha^{\prime} \alpha^{-1} \alpha^{\prime-1}$ is a nondegenerate segment.

Proposition $3.10 \quad$ 1. The group $H$ has an invariant line in $T$, and there is a short exact sequence

$$
1 \rightarrow \operatorname{Ker}\left(H \curvearrowright T_{H}\right) \rightarrow H \rightarrow \mathcal{A} \rightarrow 1
$$

where $\mathcal{A}$ contains a free abelian subgroup of index 2.

2. Let $\lambda$ be a leaf in $C(Y)$, and let $\pi$ be the image of $\pi_{1}(\lambda)$ in $H$. Then we have the following short exact sequence

$$
1 \rightarrow K \rightarrow \pi \rightarrow \Phi \rightarrow 1
$$

where $K$ fixes an arc in $T$ and $\Phi$ is a group of order at most 2.

\section{Proof.}

1. Let $A, A^{\prime}$ be the bands as in the previous lemma, and let $\alpha, \alpha^{\prime}$ denote the elements of $H$ corresponding to $A, A^{\prime}$. Let $f(J)$ be the image of $J$ in $T$ such that $\alpha, \alpha^{\prime}$ are partial orientation-preserving isometries of $f(J)$. Then the commutator $\left[\alpha, a^{\prime}\right]$ fixes a nondegenerate subarc of $f(J)$, and so fixes $T_{H}$ by Theorem 3.3. We conclude that $\alpha, \alpha^{\prime}$ are hyperbolic isometries with the same axis $L$. Let $F$ be the group generated by $\alpha, \alpha^{\prime}$. Lemma 3.6 yields that the ratio of the translation lengths of $\alpha$ and $\alpha^{\prime}$ is irrational. Thus, there are nontrivial translations with arbitrarily small translation lengths. By Proposition 3.5, $H$ is generated by $J$-short elements. Let $\gamma$ be one of generators. We need to show that $L$ is invariant under $\gamma$. Let $s \subset$ 
$f(J) \cap A x i s(\gamma)$ be a subarc such that $\gamma(s) \subset f(J) \cap A x i s(\gamma)$ (note that $f(J) \cap \operatorname{Axis}(\gamma)$ is nondegenerate because $\gamma$ is a $J$-short element).

Case I : Supposc that the partial isometry of $f(J)$ induced by $\gamma$ is orientationpreserving. Then $\gamma$ is hyperbolic and its axis contains $s \cup \gamma(s)$ by Lemma 3.1. let $\varepsilon$ be the length of the smallest connected segment containing $s \cup \gamma(s)$ in $f(J)$. Choose $w \in F \backslash\{1\}$ with small translation length so that $l(w)+$ $l(\gamma)<\varepsilon$. Then Corollary 3.1 yields that $\operatorname{Axis}(\gamma)=\operatorname{Axis}(w)$. Note that $\operatorname{Axis}(w)=L$. So $\operatorname{Axis}(\gamma)=L$ and thus $\gamma$ fixes $L$ pointwise.

Case II : Suppose that the partial isometry of $f(J)$ induced by $\gamma$ is reversing orientation. Let $m$ and $n$ be the midpoints of $s$ and $\gamma(s)$, respectively. Let $t$ denote the subinterval of $\gamma(s)$ centered at $n$ whose length is half of the length of $s$. Choose $w \in F \backslash\{1\}$ whose translation length is less than the length of $t$. Thus $w^{\prime}=\gamma w \gamma^{-1}$ is a partial orientation-preserving isometry of $f(J)$ mapping $t$ into $\gamma(s)$. Again by Corollary 3.1, the elements $w, w^{\prime}$ have the same axis $L$. Note that $\gamma \operatorname{Axis}(w)=A x i s\left(\gamma w \gamma^{-1}\right)$. It follows that $\gamma$ fixes $L$ pointwise.

This immediately implies the short exact sequence. Since $\mathcal{A} \cong H / \operatorname{Ker}(H \curvearrowright$ $\left.T_{H}\right) \supset\left\langle\alpha, \alpha^{\prime} ;\left[\alpha, \alpha^{\prime}\right]\right\rangle$, we have $\mathcal{A}$ contains a free abelian subgroup. Note that $\left|\mathcal{A}:\left\langle\alpha, \alpha^{\prime} ;\left[\alpha, \alpha^{\prime}\right]\right\rangle\right|=2$.

2. Since $\pi$ fixes a point $x$ in $T$ and the preimage of $x$ in $J$, we have that $\pi$ fixes $x$ in $L$. Let $\pi^{\prime}$ be the subgroup of index at most 2 in $\pi$ that consists of elements preserving orientation on $L$. Then $\pi^{\prime}$ acts trivially on $L$. Let $K=\pi^{\prime}$ and $\Phi \cong \pi / K$. This concludes the proof.

\subsubsection{The thin case}

Let $C(Y)$ be the thin case. Then we have an infinite sequence of band complexes $X_{0}, X_{1}, \cdots$ obtained by applying Process I recursively to the minimal component $C(Y)$.

Proposition 3.11 Under the above conditions, the group $G$ splits over a subgroup that fixes an arc in $T$. If $C(Y)$ has no annuli, then the decomposition is a free product.

ProOF. Let $\mathcal{A}_{n}$ denote the set of subdivision annuli in $X_{n}$. By Proposition 3.6, eventually there is a band $B_{n}=b_{n} \times I$ that looks like Figure 34:

Let $h_{n}=b_{n} \times\left\{\frac{1}{2}\right\}$ be the horizontal segment in $B_{n}$, and let $A_{1, n}, \cdots, A_{m, n} \in \mathcal{A}_{n}$ be the subdivision annuli intersecting $B_{n}$ in the end points of $h_{n}$. Note that $A_{j, n}$ does not have to be annulus in $X_{n}$.

If it does, then each is of the form

$$
\alpha_{j, n}: S^{1} \times I \rightarrow A_{j, n} \subset X_{n}
$$




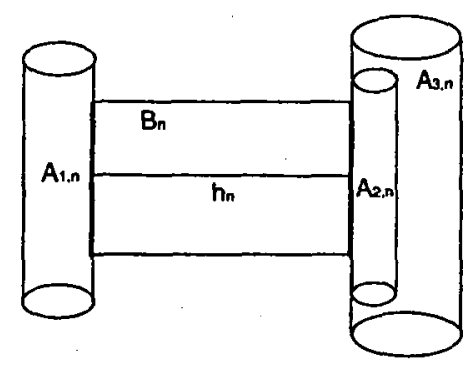

Figure 34: The band $B_{n}$ with subdivision annuli $A_{1, n}, A_{2, n}, A_{3, n}$.

for $\alpha_{j, n}$ a continuous map. Let $\pi: S^{1} \times I \rightarrow I$ be projection onto $I$. So for each $j$, there is a collection of horizontal circles $C_{j, n}=\pi^{-1}\left(\pi\left(\alpha_{j, n}^{-1}(\partial h)\right)\right)$ in $S^{1} \times I$. We retain the notation $C_{j, n}$ for the homeomorphic image of $C_{j, n}$ in $X_{n}$. Then $\Lambda_{n}=h_{n} \cup C_{1, n} \cup \cdots \cup C_{m, n}$ is a finite graph consisting of two bouquets of circles connected by an edge, and locally separates $X_{n}$. Figure 34 turns out :

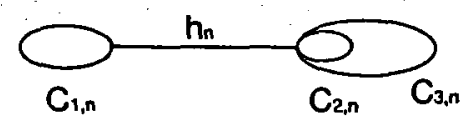

Figure 35: The graph corresponding to Figure 34.

So $X_{n}$ can be thought of as a total space with edge space $\Lambda_{n}$ and vertex spaces the closure of the components $X_{n} \backslash \Lambda_{n}$. This defines a splitting of the fundamental group $G$ of $X_{n}$. We need to show that the splitting is non-trivial.

Since the generators of the fundamental groups of the circles $C_{j, n}$ fix an arc in $T_{H}$, Theorem 3.3 implies that the edge group $\pi_{1}\left(\Lambda_{n}\right)$ in $G$ fixes $T_{H}$. So $\pi_{1}\left(\Lambda_{n}\right)$ fixes an $\operatorname{arc}$ in $T$.

If $X_{n} \backslash \Lambda_{n}$ has only one component, $G$ splits as an HNN-extension and we are done. So we may assume that $X_{n} \backslash \Lambda_{n}$ has two components $X_{n}^{\prime}$ containing $b_{n}$ and $X_{n}^{\prime \prime}$ containing $b_{n}^{*}$. Since by applying Process I further would eventually divide $B_{n}$ into many bands and the number of long bands is bounded above, there are paths $p^{\prime}: I \rightarrow X_{n}^{\prime}, p^{\prime \prime}: I \rightarrow X_{n}^{\prime \prime}$ and long bands $B_{n}^{\prime}, B_{n}^{\prime \prime}$ such that

1. $p^{\prime}(I), p^{\prime \prime}(I)$ are contained in non-sigular leaves $L^{\prime}, L^{\prime \prime}$ respectively;

2. $L^{\prime} \subset B_{n}^{\prime}, L^{\prime \prime} \subset B_{n}^{\prime \prime}$;

3. $p^{\prime}(I) \cap B_{n} \subset b_{n}, p^{\prime \prime}(I) \cap B_{n} \subset b_{n}^{*}$ each consists of two distinct points.

Therefore, both paths have non-trivial holonomy, and so each vertex group acts non-trivially on $T$ by Remark 3.3. Note that the edge group $\pi_{1}\left(\Lambda_{n}\right)$ fixes $T$ by Theorem 3.2. Thus, the edge group is a proper subgroup of each of the vertex subgroups. This implies that the splitting of $G$ is non-trivial.

If each subdivision annuli $A_{j, n}$ is not an annulus, then we can project $A_{j, n}$ onto segments $C_{j, n}$. Then $\Lambda_{n}=h_{n} \cup C_{1, n} \cup \cdots \cup C_{m, n}$ is a finite graph consisting of two bouquets of segments connected by an edge, and locally separates $X_{n}$. See Figure 


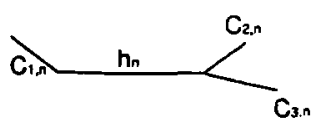

Figure 36: The graph corresponding to each subdivision annuli is not an annulus.

36. Note that the fundamental group of $\Lambda_{n}$ is trivial. Hence, the decomposition of $G$ is a free product.

\subsection{The decomposition theorem}

Let $G$ be a finitely presented group with a stable action on an $\mathbb{R}$-tree $T$.

THEOREM 3.4 Under above conditions, the following are satisfied :

1. If the action of $G$ on $T$ is pure, then $G$ satisfies one of the following cases :

(a) Surface case:

$$
1 \rightarrow K \rightarrow G \rightarrow \pi_{1}(O) \rightarrow 1,
$$

where $K$ is the kernel of the action of $G$ on $T$ and $O$ is $\bar{Y}$ with some 2-cells attached.

(b) Toral case : $T$ is a line and

$$
1 \rightarrow K \rightarrow G \rightarrow A \rightarrow 1
$$

where $K$ is the kernel of the action of $G$ on $T$ and $A$ is a subgroup of $\operatorname{Isom}(\mathbb{R})$.

(c) Thin case : $G$ splits over a subgroup that fues an arc of $T$.

2. If the action of $G$ on $T$ is not pure, then $G$ splits (as an amalgamated free product or $H N N$ extension) over a subgroup $E$ which has a short exact sequence

$$
1 \rightarrow K_{E} \rightarrow E \rightarrow C \rightarrow 1,
$$

where $K_{E}$ fixes an arc in $T$ and $C$ is either finite or cyclic. The subgroup $E$ fixes a point in $T$.

Proof. We can construct a resolving band complex $X$ by Theorem 3.1. Applying Lemma 3.2 to $X$, we may assume that $X$ has no faults. Then we apply the Rips machine to each component $C(Y)$ of the underlying union of bands $Y$.

1. If the action is pure, then $C(Y)$ is minimal. The results (a), (b) and (c) follows immediately from Proposition 3.9, 3.10, and 3.11, respectively. 
2. If the action is not pure and no component of $Y$ in the thin case, then $G$ has a graph of groups decomposition that is given by a total space decomposition of $X$ Theorem 3.2. Suppose that the decomposition is non-trivial. Let $E$ be an edge group of the graph of groups decomposition of $G$. Then $G$ splits over $E$ and $E$ fixes a point in $T$. Hence $E$ is contained in the image of the fundamental group of some leaf in some component $C(Y)$.

If $C(Y)$ is minimal (i.e., surface case or toral case, but not thin case), then $E$ satisfies the assertion of the theorem by Proposition 3.9 and 3.10 .

By Proposition 3.3, we may assume that $C(Y)$ is simplicial now. Then $C(Y)$ is an $I$-bundle over a leaf. Consider a finite covering $\widetilde{C(Y)}$ of $C(Y)$. Thus $\widetilde{C(Y)}$ is a $I$-bundle over a leaf with the trivial fundamental group. Then its fundamental group fixes an arc in $T$ and so $E \cong K_{E}$. It implies that $C$ is finite. The result follows.

\section{Conclusions}

We exploit and generalize the properties of two older group-theoretic constructions: free product with amalgamation and HNN extension. However, unlike the traditional algebraic study of these two constructions, we use the geometric language of covering theory and fundamental groups. Graphs of groups, which are the basic objects of this article, can be viewed as one-dimensional $\mathrm{CW}$-complexes. By using those technique, we discover the algebraic structure of subgroups of free products, amalgamated free products and HNN extensions. The basic treatment of this section is available in the article of [SW79] and the book of [Coh78].

In Rips theory, we have a stable action $G$ (finitely presented) on an $\mathbb{R}$-tree $T$; then we construct a band complex $X$ resolving the action on $T$. The band complex $X$ breaks into a union of subcomplexes of simplicial and pure type. Applying each simplicial or pure component of $X$ to the Rips machine, we have a class of band complexes which are easily manipulated. It turns out that the action $G$ on $T$ can be easily understood now. This section mainly follows from the articles of [Kap01] and [Wil03]. Another reference is given by [BF95]. 


\section{References}

[BF95] Mladen Bestvina and Mark Feighn. Stable actions of groups on real trees. Invent. Math., (121):287-320, 1995.

[CGKZ98] D. J. Collins, R. I. Grigorchuk, P. F. Kurchanov, and H. Zieschang. Combinatorial group theory and applications to geometry. Springer, Berlin; New York, 1998.

[Coh78] Daniel E. Cohen. Combinatorial group theory, a topological approach. Queen Mary College mathematics notes. Queen Mary College, Dept. of Pure Mathematics, London, 1978.

[Hat02] Allen Hatcher. Algebraic Topology. Cambridge University Press, Cambridge, England, 2002.

[Kap01] Michael Kapovich. Hyperbolic manifolds and discrete groups, chapter 12, Rips Theory, pages 279-321. Progress in mathematics ; v. 183. Birkhäuser, Boston, 2001.

[Lec07] Graphs and group actions, Winter 2007. Lecture notes Math $4106 / 5106$ offered in Carleton university. Available at http://www . math. carleton. ca/ bumagin/teaching/4106/math4106.html.

[LS77] Roger C. Lyndon and Paul E. Schupp. Combinatorial group theory. Ergebnisse der Mathematik und ihrer Grenzgebiete; 89. SpringerVerlag, Berlin; New York, 1977.

[Mas67] William S. Massey. Algebraic topology: an introduction. Harbrace college mathematics series. Harcourt, Brace and World, New York, 1967.

[Mun00] James R. Munkres. Topology, Second Edition. Prentice-Hall, Englewood Cliffs, N. J., 2000.

[Ser03] Jean-Pierre Serre. Trees. Springer-Verlag, Berlin, Heidelberg, New York, corrected second printing edition, 2003.

[SW79] Peter Scott and Terry Wall. Homological group theory, chapter 5; Topological methods in group theory, pages 137-165. London Mathematical Society lecture note series; 36. Cambridge University Press, Cambridge, England, 1979.

[Wil03] Henry Wilton. Rips theory, April 2003. Available at http://www. math.utexas.edu/users/henry.wilton/.

[Wil04] Henry Wilton. Group actions on trees, November 2004. Available at http://www . math . utexas . edu/users/henry.wilton/trees.pdf. 